\title{
A study of tubular string buckling in vertical wells
}

\author{
Wenjun Huang ${ }^{\mathrm{a}, \mathrm{b}}$, Deli Gao ${ }^{\mathrm{b}}$, Yinghua Liu ${ }^{\mathrm{a}}$ \\ ${ }^{a}$ School of Aerospace Engineering, AML, Tsinghua University, Beijing 100084, China \\ ${ }^{b}$ MOE Key Laboratory of Petroleum Engineering, China University of Petroleum, Beijing 102249, China \\ *Corresponding author: huangwenjun1986@126.com, gaodeli@ cast.org.cn, yhliu@ tsinghua.edu.cn
}

\begin{abstract}
A new model depicting the full phase of tubular string buckling in vertical wellbores is proposed. The buckling modes of the tubular string are divided into: no buckling, 2D lateral buckling, 3D lateral buckling, continuous contact buckling and helical buckling. For the 2D lateral and 3D lateral buckling, the tubular string is respectively depicted by two and four suspended sections; for the continuous contact and helical buckling, the tubular string includes four suspended sections and one continuous contact section. With the suspended and continuous contact sections respectively depicted by beam-column model and buckling differential equation, the general buckling configurations of the entire tubular string are deduced. Substituting relevant continuity, boundary and stability conditions, the tubular string buckling problem is converted into a system of nonlinear equations. Solving these equations with iteration method, the critical buckling loads and the buckling configurations under different buckling modes for a weightless tubular string are obtained. Later, the weightless model is extended to the case for a tubular string with weight. On the basis of the new buckling model, the axial force and torque transfer along the tubular string under the coupling effect of friction force and buckling is discussed. The results show that the existence of tubular string weight increases the complexity of buckling mechanism and calculation. The continuous contact between the tubular string and wellbore reduces the axial force and torque transfer along the tubular string. To increase the axial force and torque transfer and inhibit buckling, the installation of stabilizers of low friction and buckling inhibition on the buckling-prone sections is wiser than unilaterally increasing the diameter and thickness of the tubular string. Compared with the previous results, the new model provides a more sophisticated description of tubular string buckling in vertical wellbores.
\end{abstract}

Keywords: drilling engineering; tubular mechanics; tubular string; buckling

\section{Introduction}

Tubular string buckling is an important issue in well engineering. Buckling can make the tubular string deviate from its initial straight state and deform into a curved configuration, which may further lead to a series of drilling problems, such as well deviation, tubular string failure, casing wear, "lock up", etc. The tubular string buckling problem is analogous to the buckling of the Euler rod with compressions on its ends. However, the tubular string buckling in well engineering is more complex since other factors are involved such as wellbore constraint, tubular gravity, boundary conditions, friction force, torque, and so on. Until now, a lot of research on tubular string buckling has been done and newly results are continually published to promote the understanding of tubular string buckling (Hajianmaleki, 2014). In brief, the previous studies have developed from the following aspects(Gao and Huang, 2015): (1) Critical buckling loads and buckling configurations under different buckling modes in vertical(Lubinski, 1950; Mitchell, 1988; Huang and Gao et al., 2015), horizontal, straightly inclined(Paslay and Bogy, 1964; Wu, 1992; Tan, 1993; Mitchell, 1997; Huang and Gao et al., 2015) and curved wellbores(Wu and Juvkam-Wold, 1995; Mitchell, 1999); (2) The effects of torque, boundary condition(Mitchell, 1982; Sorenson and Cheatham, 1986; Huang and Gao et al., 2015), friction force, connectors(Mitchell, 2003; Mitchell and Stefan, 2006; Huang and Gao, 2014; Huang and Gao, 2014; Huang and Gao, 2015), etc; (3) Research methods including beam-column model(Lubinski, 1977), buckling differential equation(Mitchell, 
1988; Huang and Gao et al., 2015) and energy method(Chen and Lin et al., 1990; Wu, 1992; Cunha, 2004). In this paper, we only focus on the tubular string bucking in vertical wellbore.

Lubinski (1950) firstly conducted a systematic study on the two-dimensional (2D) lateral buckling of a tubular string in the vertical wellbores. At the initial state, the entire tubular string is hanging on the well head and the tubular string remains in the initial straight line configuration. During the tripping in process by ground hook, the weight on bit (WOB) increases. When WOB achieves a certain value, the straight line configuration become unstable, and the tubular string laterally buckles into a $2 \mathrm{D}$ curve and touches the inner surface of the wellbore at one single point. If the WOB further increases and exceeds another critical value, the tubular string buckles in a second-order 2D curved configuration. At this point, the tubular string tends to touch the both sides of the wellbore. The critical WOBs for the first- and second-order buckling loads are respectively given in Table 1 . The tubular string may buckle into a third order 2D helical buckling configuration if the WOB is continually increased. Later, Lubinski and Althouse (1962) pointed out that the tubular string does not sequentially buckle into a higher-order 2D lateral buckling configuration but becomes into a three-dimensional (3D) buckling configuration with the increase of WOB. It was assumed that the tubular string is in continuous contact with the wellbore and the deflection curve of the tubular string is a helix, the pitch-force relationship is deduced with energy method:

$$
F=\frac{8 \pi^{2} E I}{p^{2}}
$$

Where $F$ is the axial compressive force, $E I$ is the bending stiffness of the tubular string and $p$ is the pitch of helix.

In actual application, the neutral point $(F=0)$ is usually considered to be the starting point of helical buckling in vertical wellbore. As a result, the down-hole tubular string deflection is divided into two parts with the neutral point as the dividing point: the top suspended section and the bottom continuous contact section.

However, taking the neutral point as the critical buckling load is too conservative for large-diameter and high-thickness tubular strings, such as drill collar. Later, $\mathrm{Wu}(\mathrm{Wu}, 1992)$ deduced the critical sinusoidal buckling and helical buckling loads in vertical wellbores with the energy method shown in Table 1. In Wu's analysis, the entire tubular string is assumed to be in continuous contact with the wellbore, and the critical sinusoidal buckling and helical buckling conditions are obtained when the tubular string buckles into a half-sine wave and a pitch of helix. Gao (Gao, 2006) proved that the helical buckling in vertical wellbores is stable while the sinusoidal buckling is stable. In Gao's analysis, the helical buckling solutions in inclined wellbores are calculated on the basis of buckling differential equation, and the critical helical buckling force is defined as the minimum axial force under which the contact force between the tubular string and the top of the wellbore is 0 . Then, the critical contact force in inclined wellbores is extended to that in vertical wellbore by assuming that the length of tubular string which continuously touches the wellbore is at least a period of helix. Although different research methods are adopted in Wu's and Gao's analyses, their results are rather close to each other quantitatively. However, the assumption of continuous contact between the entire tubular string and the wellbore is not satisfied, since the top portion of the tubular string near the well head is in no contact with the wellbore.

Table 1-The critical buckling loads for vertical wellbores

\begin{tabular}{cccc}
\hline Buckling mode & 2D Lateral buckling & Sinusoidal buckling & Helical buckling \\
\hline Dimensionless force & & & 0.00 (Lubinski and \\
$\left(F / \sqrt[3]{E I q^{2}}\right)$ & 1.94 and 3.75 (Lubinski, & $2.55(\mathrm{Wu}, 1992)$ & Althouse, 1962) \\
& $1950)$ & & $5.55(\mathrm{Wu}, 1992)$ \\
& & $5.62(\mathrm{Gao}, 2006)$ \\
\hline
\end{tabular}

Mitchell (Mitchell, 1988) calculated the helical bucking solution with buckling differential equation and proved that 
Lubinski's result (Lubinski and Althouse, 1962) was just an approximate result under a large WOB. With Eq. (1), the contact force between the tubular string and wellbore is obtained (Mitchell, 1988):

$$
N=\frac{F r_{c}^{2}}{4 E I}
$$

Where $N$ is the contact force per unit length of the tubular string and $r_{c}$ is the radial clearance between the tubular string and wellbore.

On the basis of the above buckling models, Mitchell (Mitchell, 1986) deduced the axial force transfer along the tubular string in slack off operation in vertical wellbores, in which the neutral point is considered to be the starting position of helical buckling section:

$$
F=\sqrt{\frac{4 E I q}{\mu r_{c}}} \tanh \left(z \sqrt{\frac{\mu q r_{c}}{4 E I}}+c\right)
$$

Where $z$ is the axial distance along the wellbore, $q$ is tubular string weight per unit length, $\mu$ is the friction factor and $c$ is an integral constant.

Eq. (3) indicates that the axial force on the helical buckling section increases in a hyperbolic tangent function with respect to the vertical distance and approaches the upper limit value of $\sqrt{\frac{4 E I q}{\mu r_{c}}}$ when the helically buckled section tends to infinity. Later, Mitchell (Mitchell, 1996) deduced the axial displacement-based governing equation with friction force and calculated the axial force transfer with finite element method. Wu and Juvkam-Wold (Wu and Juvkam-Wold, 1995) further developed Mitchell's model and deduced the axial force transfer (Eq. (3)) with the critical helical buckling load given in Table 1 as the starting point of helical buckling section. Gulyayev (2009) studied the buckling of tubular string under the actions of torque, gravity and centrifugal inertia forces generated by moving flows of internal mud and rotating masses of the tubular string, and the results show that torque can cause incipient buckling mode representing superposition of spiral curves and short length wavelets in the bottom of the tubular string. Recently, Mitchell (Mitchell, 2000; Mitchell and Stefan, 2006) and Huang (Huang and Gao, 2014) studied the helical buckling behavior of a tubular string with connectors in vertical wellbores.

The above studies can be summarized into three important issues: (1) the transition from 2D lateral buckling to 3D helical buckling with the increase of WOB; (2) the transition between the top suspended section and bottom helically buckled section at the helical buckling stage; (3) the axial force and torque transfer along the tubular string. For the first issue, the previous studies respectively provide the values of critical 2D lateral buckling and helical buckling loads. Since the critical buckling loads in Table 1 are deduced on different assumptions and calculated by different methods, each buckling load only represents a special situation which not usually conflicts with other critical values. That is to say, these buckling loads cannot be combined into a whole model to depict the entire buckling transition process. For the second issue, the previous models focus on the deflection behaviors of the helical buckling section. Meanwhile, the starting point of helical buckling was approximately considered to be the critical helical buckling loads in Table 1. Thus, the deflection of the suspended section and continuous contact section was not taken as a whole. The studies on the third issue were based on the former two issues, so a more accurate model of axial force and torque transfer needs to be built.

To cope with these problems, a more sophisticated buckling model for a tubular string in vertical wellbores is proposed in this paper. Firstly, a full buckling model for a weightless tubular string in vertical wellbores is built, where the suspended section is depicted by beam-column model and the continuous contact section by the buckling differential equation. Next, the weightless model is extended to the case for a tubular string with weight. The critical buckling loads and buckling configurations for 2D lateral buckling, 3D lateral buckling, continuous contact buckling and helical buckling are respectively calculated. On the basis of the new model, the axial force and torque transfer along the tubular string under the coupling effect of friction force and buckling is discussed at last. 


\section{Buckling model of weightless tubular strings}

\subsection{Model description}

For a compressed weightless tubular string constrained in a vertical wellbore, the entire process of the buckling mode transition is shown in Fig. 1. In the initial state (Fig. 1a), the tubular string keeps its initial non-buckling straight configuration. When the axial force exceeds a certain value, the straight configuration becomes unstable and the tubular string buckles into a curved configuration (Fig. 1b) in a two-dimensional (2D) plane. In the 2D lateral buckling state, the middle of the tubular string touches the inner surface of the wellbore. With increasing axial force, the lateral displacement also increases. When the axial force reaches another critical value, the 2D lateral buckling state becomes unstable and the tubular string buckles in a 3D curved configuration (Fig. 1c). In the 3D lateral buckling state, the tubular string touches the wellbore at three contact points which divides the deflection of the tubular string into four suspended sections (Fig. 1d). When the axial force further exceeds the third critical value, the contact point on the middle of the tubular string vanishes and a small portion of the tubular string starts to be in continuous contact with the wellbore (Fig. 1e). In the 3D continuous contact buckling state, the total tubular string is divided into four suspended sections and one continuous contact section. When the length of the continuous contact section increases to one pitch of helix (Fig. 1f), the axial force is taken as the critical helical buckling load (Gao, 2006).

The above analyses only qualitatively depict the entire transition process of tubular string buckling mode. The quantitative results about the critical loads between different buckling modes and the buckling configurations under all the buckling modes should be given. To achieve this objective, the following assumptions are adopted in the following analyses:

1. The axis of the wellbore is a straight line and the cross section is a circle.

2. The radial clearance between the tubular string and the wellbore is far lower than the axial length of the tubular string, so the lateral deformation of the tubular string is small and in elastic range.

3. The two ends of the tubular string are hinged supports on the axis of the wellbore.

4. Friction, torque, rotation and weight are neglected in this section. 


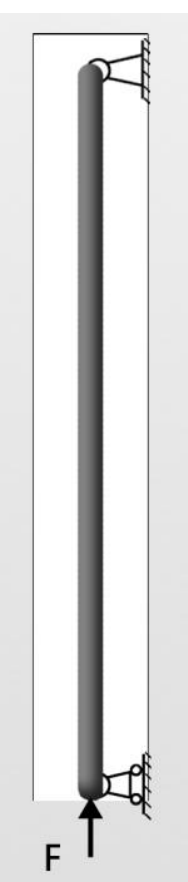

(a)

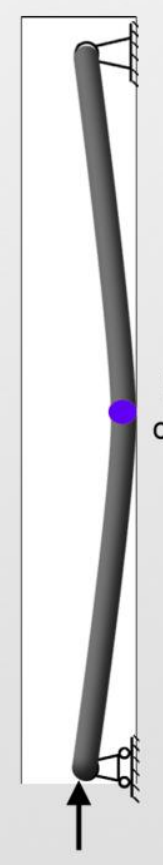

(b)

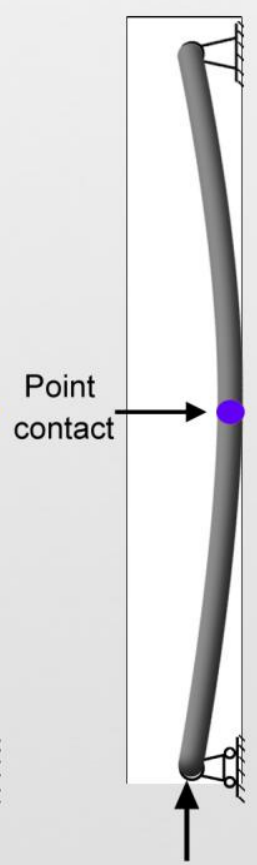

(c)

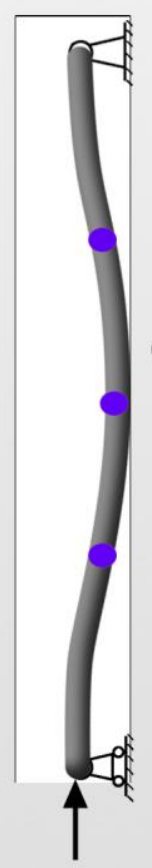

(d)

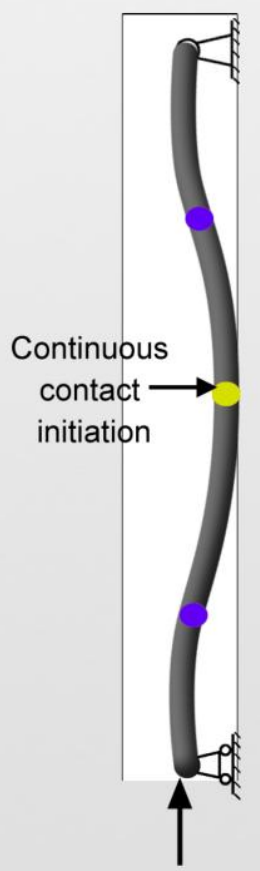

(e)

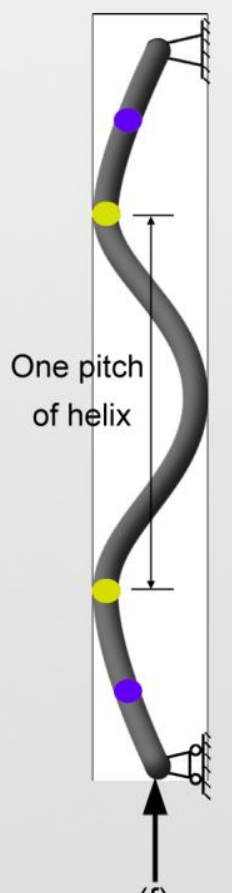

(f)

Fig. 1. Buckling mode transition for a weightless tubular string constrained in a straight wellbore: (a) initial straight configuration, (b) critical 2D lateral buckling, (c) critical 3D lateral buckling, (d) 3D lateral buckling, (e) critical continuous contact buckling and (f) critical helical buckling.

\subsection{Non-buckling to 2D lateral buckling}

The deflection curve on the top half part of a weightless tubular string constrained in a vertical wellbore is shown in Fig. 2. The dimensionless distance along the wellbore axis is defined by

$$
\eta=\sqrt{\frac{F}{2 E I}} \cdot z
$$

Where $F$ is the axial compression, EI is the bending stiffness of the tubular string, $z$ is the axial distance. The dimensionless lateral displacements of the tubular string are defined by

$$
\mu=\frac{y}{r_{c}} \quad v=\frac{x}{r_{c}}
$$

Where $x$ and $y$ are the lateral displacements and $r_{c}$ is the radial clearance between the tubular string and the wellbore.

Timoshenko and Gere (1963) deduced the weightless beam-column model as follows:

$$
E I \frac{d^{4} w}{d z^{4}}+F \frac{d^{2} w}{d z^{2}}=0
$$

Where $w=x, y$ represents the lateral displacement along $\mathrm{x}$-axis or $\mathrm{y}$-axis.

By introducing the dimensionless terms Eq. (4) and (5), the above equation can be expressed in the dimensionless form:

$$
\frac{d^{4} \mu}{d \eta^{4}}+2 \frac{d^{2} \mu}{d \eta^{2}}=0 \quad \frac{d^{4} v}{d \eta^{4}}+2 \frac{d^{2} v}{d \eta^{2}}=0
$$

The general solution for Eq. (7) is expressed by

$$
\mu=\sum_{i=1}^{4} c_{i} \cdot d_{i}(\eta) \quad v=\sum_{i=1}^{4} c_{i+4} \cdot d_{i}(\eta)(8)
$$


Where the dimensionless displacements in direction $\mathrm{x}$ and $\mathrm{y}$ are shown by both $v$ and $\mu$. And the first, second and third derivatives of Eq. (8) with respect to $\eta$ are given below:

$$
\begin{aligned}
\frac{d \mu}{d \eta} & =\sum_{i=1}^{4} c_{i} \cdot e_{i}(\eta) \quad \frac{d v}{d \eta}=\sum_{i=1}^{4} c_{i+4} \cdot e_{i}(\eta) \\
\frac{d^{2} \mu}{d \eta^{2}} & =\sum_{i=1}^{4} c_{i} \cdot f_{i}(\eta) \quad \frac{d^{2} v}{d \eta^{2}}=\sum_{i=1}^{4} c_{i+4} \cdot f_{i}(\eta) \\
\frac{d^{3} \mu}{d \eta^{3}} & =\sum_{i=1}^{4} c_{i} \cdot g_{i}(\eta) \frac{d^{3} v}{d \eta^{3}}=\sum_{i=1}^{4} c_{i+4} \cdot g_{i}(\eta)
\end{aligned}
$$

Where $c_{i}$ are the integral constants and $d_{i}, e_{i}, f_{i}$ and $g_{i}$ are calculated by

$$
\begin{array}{llll}
d_{1}(\eta)=\cos (\sqrt{2} \eta) & d_{2}(\eta)=\sin (\sqrt{2} \eta) & d_{3}(\eta)=\eta & d_{4}(\eta)=1 \\
e_{1}(\eta)=-\sqrt{2} \sin (\sqrt{2} \eta) & e_{2}(\eta)=\sqrt{2} \cos (\sqrt{2} \eta) & e_{3}(\eta)=1 & e_{4}(\eta)=0 \\
f_{1}(\eta)=-2 \cos (\sqrt{2} \eta) & f_{2}(\eta)=-2 \sin (\sqrt{2} \eta) & f_{3}(\eta)=0 & f_{4}(\eta)=0 \\
g_{1}(\eta)=2 \sqrt{2} \sin (\sqrt{2} \eta) & g_{2}(\eta)=-2 \sqrt{2} \cos (\sqrt{2} \eta) & g_{3}(\eta)=0 & g_{4}(\eta)=0
\end{array}
$$

Here we let $\eta_{0}=\sqrt{\frac{F}{2 E I}} \cdot 0=0$ denote the left end of the tubular string and $\eta_{L}=\sqrt{\frac{F}{2 E I}} \cdot L$ denote the right end of the tubular string, where $L$ is the length of the entire tubular string. Note that, the parameter $\eta_{L}$ can also be taken as the dimensionless axial compression. For the non-buckling to 2D lateral buckling case, the lateral deflection on the coordinate plane of $o-\eta-\mu$ is considered. The boundary conditions on the two ends are given as follows:

(1) The bending moments on the two ends $\left(\eta=\eta_{0}\right.$ and $\left.\eta=\eta_{L}\right)$ are both equal to 0 .

(2) The lateral displacements on the two ends $\left(\eta=\eta_{0}\right.$ and $\left.\eta=\eta_{L}\right)$ are both equal to 0 .

Substituting the general solution in Eq. (8)-(11) into the above boundary conditions, we obtain the following set of 4 equations:

$$
\left(\begin{array}{llll}
f_{1}\left(\eta_{0}\right) & f_{2}\left(\eta_{0}\right) & f_{3}\left(\eta_{0}\right) & f_{4}\left(\eta_{0}\right) \\
f_{1}\left(\eta_{L}\right) & f_{2}\left(\eta_{L}\right) & f_{3}\left(\eta_{L}\right) & f_{4}\left(\eta_{L}\right) \\
d_{1}\left(\eta_{0}\right) & d_{2}\left(\eta_{0}\right) & d_{3}\left(\eta_{0}\right) & d_{4}\left(\eta_{0}\right) \\
d_{1}\left(\eta_{L}\right) & d_{2}\left(\eta_{L}\right) & d_{3}\left(\eta_{L}\right) & d_{4}\left(\eta_{L}\right)
\end{array}\right)\left(\begin{array}{l}
c_{1} \\
c_{2} \\
c_{3} \\
c_{4}
\end{array}\right)=\left(\begin{array}{l}
0 \\
0 \\
0 \\
0
\end{array}\right)
$$

Since the right hand of Eq. (13) is a vector of zero, the solutions of Eq. (13) have physical meanings only if the determinant of the coefficient matrix on the left hand is zero.

$$
\left|\begin{array}{llll}
f_{1}\left(\eta_{0}\right) & f_{2}\left(\eta_{0}\right) & f_{3}\left(\eta_{0}\right) & f_{4}\left(\eta_{0}\right) \\
f_{1}\left(\eta_{L}\right) & f_{2}\left(\eta_{L}\right) & f_{3}\left(\eta_{L}\right) & f_{4}\left(\eta_{L}\right) \\
d_{1}\left(\eta_{0}\right) & d_{2}\left(\eta_{0}\right) & d_{3}\left(\eta_{0}\right) & d_{4}\left(\eta_{0}\right) \\
d_{1}\left(\eta_{L}\right) & d_{2}\left(\eta_{L}\right) & d_{3}\left(\eta_{L}\right) & d_{4}\left(\eta_{L}\right)
\end{array}\right|=0
$$

Expression Eq. (14) gives the critical axial compression which must be satisfied for the 2D lateral buckling to occur. Substituting Eq. (12) into Eq. (14), we obtain that the critical axial compression are expressed by $\eta_{L 2 D}=\frac{\pi}{\sqrt{2}}$ or

$$
F_{2 D}=\frac{\pi^{2} E I}{L^{2}}
$$

Note that Eq. (15) is the Euler critical force for two hinged ends (Gere and Goodno, 2008).

When the axial force exceeds the critical value $F_{2 D}$, the initial straight configuration is unstable and the deflection of the tubular string starts to deviate from the wellbore axis. If the wellbore constraint is removed, the 2D lateral 
buckling of the tubular string once started would not stop. Then, the tubular string would continue to bend without achieving any equilibrium state. This problem is mainly due to the fact that Eq. (7) is only applicable for the small deflection case. By introducing the tubular string mechanics based on large deflection, this problem can be well solved. However, the small lateral deflection of the tubular string due to the constraints of the wellbore is considered here (assumption 2), so the small deflection theory Eq. (7) can meet the accuracy requirement.

At the 2D lateral buckling stage, the middle point of the tubular string touches the wellbore according to the symmetry of the deflection curve (Fig. 2b). Therefore, only the top half portion of the tubular string is considered, in which the top end is a hinged support and the middle point is a fixed support. Then, the post 2D lateral buckling configuration of the top half portion can be obtained based on the following conditions:

(1) The lateral displacement and bending moment on the top end $\left(\eta=\eta_{0}\right)$ are equal to 0 .

(2) The lateral displacement on the middle point $\left(\eta=\frac{\eta_{L}}{2}\right)$ is equal to 1 and the first derivative of the lateral displacement on the middle point is equal to 0 .

Substituting the general solution in Eq. (8)-(11) into the above conditions, we obtain the following set of 4 equations:

$$
\left(\begin{array}{llll}
f_{1}\left(\eta_{0}\right) & f_{2}\left(\eta_{0}\right) & f_{3}\left(\eta_{0}\right) & f_{4}\left(\eta_{0}\right) \\
e_{1}\left(\frac{\eta_{L}}{2}\right) & e_{2}\left(\frac{\eta_{L}}{2}\right) & e_{3}\left(\frac{\eta_{L}}{2}\right) & e_{4}\left(\frac{\eta_{L}}{2}\right) \\
d_{1}\left(\eta_{0}\right) & d_{2}\left(\eta_{0}\right) & d_{3}\left(\eta_{0}\right) & d_{4}\left(\eta_{0}\right) \\
d_{1}\left(\frac{\eta_{L}}{2}\right) & d_{2}\left(\frac{\eta_{L}}{2}\right) & d_{3}\left(\frac{\eta_{L}}{2}\right) & d_{4}\left(\frac{\eta_{L}}{2}\right)
\end{array}\right)\left(\begin{array}{l}
c_{1} \\
c_{2} \\
c_{3} \\
c_{4}
\end{array}\right)=\left(\begin{array}{l}
0 \\
0 \\
0 \\
1
\end{array}\right)
$$

Solving the constants $c_{i}$ from Eq. (16) and substituting them into Eq. (8), the deflection curve of the tubular string can be determined.

\subsection{D lateral buckling to 3D lateral buckling}

The above analyses only consider the 2D lateral buckling in the coordinate plane $o-\eta-\mu$. However, when the axial force reaches a certain value, the 2D lateral buckling configuration becomes unstable and the buckling begins to appear in the coordinate plane o- $\eta-v$. At the 2D lateral buckling stage, the following conditions on the coordinate plane o- $\eta-v$ are satisfied:

(1) The bending moments on the two ends $\left(\eta=\eta_{0}\right.$ and $\left.\eta=\eta_{L}\right)$ are equal to 0 .

(2) The lateral displacements on the two ends $\left(\eta=\eta_{0}\right.$ and $\left.\eta=\eta_{L}\right)$ are equal to 0 .

(3) The lateral displacement on the middle point $\left(\eta=\frac{\eta_{L}}{2}\right)$ is equal to 0 and the first and second derivatives of the lateral displacements are continuous at the middle contact point.

It is further assumed that the deflection curve of the tubular string is anti-symmetric about the middle point, and then the bending moment at the middle point is equal to 0 . The above conditions on the entire tubular string can be converted to that on the top half portion:

(1) The bending moments on the top end and middle point $\left(\eta=\eta_{0}\right.$ and $\left.\eta=\frac{\eta_{L}}{2}\right)$ are both equal to 0 .

(2) The lateral displacements on the top end and middle point $\left(\eta=\eta_{0}\right.$ and $\left.\eta=\frac{\eta_{L}}{2}\right)$ are both equal to 0 .

Note that the third condition (3) is automatically satisfied by introducing the assumption of the anti-symmetric tubular string deflection on the coordinate plane o- $\eta-v$. Substituting the general solutions in Eq. (8)-(11) into the above conditions, we obtain the following set of 4 equations: 


$$
\left(\begin{array}{llll}
f_{1}\left(\eta_{0}\right) & f_{2}\left(\eta_{0}\right) & f_{3}\left(\eta_{0}\right) & f_{4}\left(\eta_{0}\right) \\
f_{1}\left(\frac{\eta_{L}}{2}\right) & f_{2}\left(\frac{\eta_{L}}{2}\right) & f_{3}\left(\frac{\eta_{L}}{2}\right) & f_{4}\left(\frac{\eta_{L}}{2}\right) \\
d_{1}\left(\eta_{0}\right) & d_{2}\left(\eta_{0}\right) & d_{3}\left(\eta_{0}\right) & d_{4}\left(\eta_{0}\right) \\
d_{1}\left(\frac{\eta_{L}}{2}\right) & d_{2}\left(\frac{\eta_{L}}{2}\right) & d_{3}\left(\frac{\eta_{L}}{2}\right) & d_{4}\left(\frac{\eta_{L}}{2}\right)
\end{array}\right)\left(\begin{array}{l}
c_{5} \\
c_{6} \\
c_{7} \\
c_{8}
\end{array}\right)=\left(\begin{array}{l}
0 \\
0 \\
0 \\
0
\end{array}\right)
$$

Similar to the analysis in non-buckling to 2D lateral buckling case, the following expression must be satisfied for the 3D lateral buckling initiation:

$$
\left|\begin{array}{llll}
f_{1}\left(\eta_{0}\right) & f_{2}\left(\eta_{0}\right) & f_{3}\left(\eta_{0}\right) & f_{4}\left(\eta_{0}\right) \\
f_{1}\left(\frac{\eta_{L}}{2}\right) & f_{2}\left(\frac{\eta_{L}}{2}\right) & f_{3}\left(\frac{\eta_{L}}{2}\right) & f_{4}\left(\frac{\eta_{L}}{2}\right) \\
d_{1}\left(\eta_{0}\right) & d_{2}\left(\eta_{0}\right) & d_{3}\left(\eta_{0}\right) & d_{4}\left(\eta_{0}\right) \\
d_{1}\left(\frac{\eta_{L}}{2}\right) & d_{2}\left(\frac{\eta_{L}}{2}\right) & d_{3}\left(\frac{\eta_{L}}{2}\right) & d_{4}\left(\frac{\eta_{L}}{2}\right)
\end{array}\right|=0
$$

Substituting Eq. (12) into Eq. (18), we obtain that the critical axial compression expressed by $\eta_{L, 3 D}=\sqrt{2} \pi$ or

$$
F_{3 D}=\frac{4 \pi^{2} E I}{L^{2}}
$$

Note that Eq. (19) is just the Euler critical force for two fixed ends (Gere and Goodno, 2008).

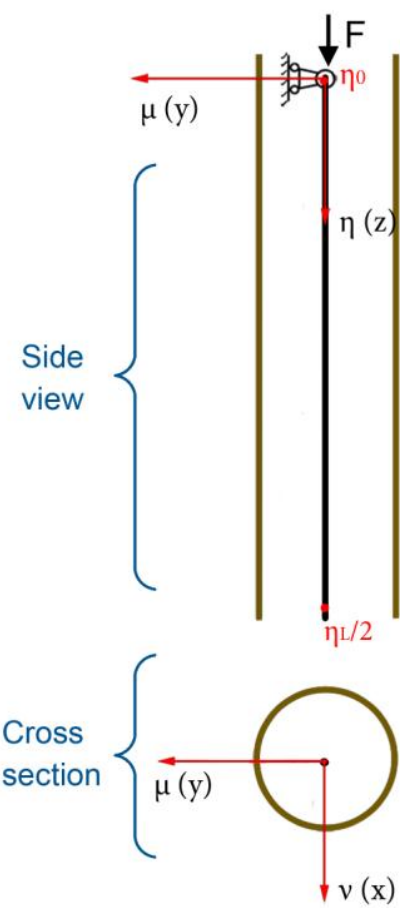

(a)
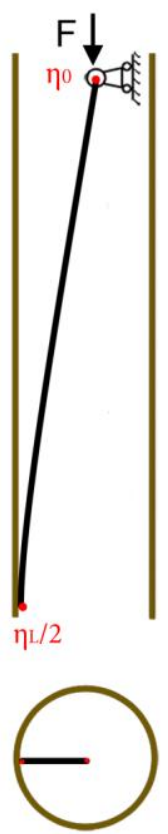

(b)
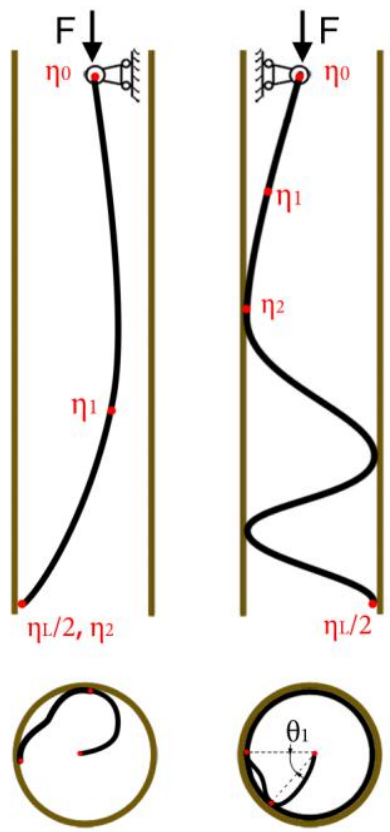

(c)

(d)

Fig. 2. Buckling configuration of a weightless tubular string constrained in a vertical wellbore

\subsection{D lateral, continuous contact and helical buckling}

With the axial force exceeding the critical load $F_{3 D}$, the tubular string buckles into 3D lateral buckling, continuous contact buckling or even helical buckling. In the 3D lateral buckling state, the top half portion of the tubular string is 
depicted by two suspended sections (Fig. 2c). If the tubular string enters into the continuous contact buckling stage, the middle portion of the tubular string becomes in continuous contact with the wellbore (Fig. 2d). Here, the suspended sections are depicted by the beam-column models and the continuous contact section is depicted by a segment of helix, namely

$$
y=r_{c} \cos \left(\kappa \cdot \sqrt{\frac{F}{2 E I}} \cdot z\right) \quad x=r_{c} \sin \left(\kappa \cdot \sqrt{\frac{F}{2 E I}} \cdot z\right)
$$

Where $\kappa$ is a constant.

The dimensionless form of Eq. (20) is expressed by

$$
\mu=\cos (\kappa \cdot \eta) \quad v=\sin (\kappa \cdot \eta)
$$

The conditions on the two suspended sections include:

(1) The lateral displacements and bending moments on the top end $\left(\eta=\eta_{0}\right)$ are equal to 0 .

(2) The lateral displacements, tangential directions and bending moments on the first contact point $\left(\eta=\eta_{1}\right)$ are continuous between the first and second suspended sections.

(3) The lateral displacements, tangential directions and bending moments on the second contact point $\left(\eta=\eta_{2}\right)$ are continuous between the second suspended section and continuous contact section.

(4) The tangential direction of the tubular string is tangent to the wellbore surface on the first contact point $\left(\eta=\eta_{1}\right)$.

The general solutions for the first and second suspended sections are respectively expressed by

$$
\begin{gathered}
\mu_{1}=\sum_{i=1}^{4} c_{i} \cdot d_{i}(\eta) \quad v_{1}=\sum_{i=1}^{4} c_{i+4} \cdot d_{i}(\eta) \\
\mu_{2}=\sum_{i=1}^{4} c_{i+8} \cdot d_{i}(\eta) \quad v_{2}=\sum_{i=1}^{4} c_{i+4+8} \cdot d_{i}(\eta)
\end{gathered}
$$

Substituting Eq. (22) into the condition (1), we obtain

$$
\begin{array}{ll}
\sum_{i=1}^{4} c_{i} \cdot d_{i}\left(\eta_{0}\right)=0 & \sum_{i=1}^{4} c_{i+4} \cdot d_{i}\left(\eta_{0}\right)=0 \\
\sum_{i=1}^{4} c_{i} \cdot f_{i}\left(\eta_{0}\right)=0 & \sum_{i=1}^{4} c_{i+4} \cdot f_{i}\left(\eta_{0}\right)=0
\end{array}
$$

Assuming that the angular displacement on the first contact point $\left(\eta_{1}\right)$ is $\theta_{1}$, we obtain the following expressions by substituting Eq. (22) and (23) into condition (2):

$$
\begin{array}{cl}
\sum_{i=1}^{4} c_{i} \cdot d_{i}\left(\eta_{1}\right)=\cos \theta_{1} & \sum_{i=1}^{4} c_{i+4} \cdot d_{i}\left(\eta_{1}\right)=\sin \theta_{1} \\
\sum_{i=1}^{4} c_{i+8} \cdot d_{i}\left(\eta_{1}\right)=\cos \theta_{1} & \sum_{i=1}^{4} c_{i+4+8} \cdot d_{i}\left(\eta_{1}\right)=\sin \theta_{1} \\
\sum_{i=1}^{4} c_{i} \cdot e_{i}\left(\eta_{1}\right)-\sum_{i=1}^{4} c_{i+8} \cdot e_{i}\left(\eta_{1}\right)=0 & \sum_{i=1}^{4} c_{i+4} \cdot e_{i}\left(\eta_{1}\right)-\sum_{i=1}^{4} c_{i+4+8} \cdot e_{i}\left(\eta_{1}\right)=0 \\
\sum_{i=1}^{4} c_{i} \cdot f_{i}\left(\eta_{1}\right)-\sum_{i=1}^{4} c_{i+8} \cdot f_{i}\left(\eta_{1}\right)=0 & \sum_{i=1}^{4} c_{i+4} \cdot f_{i}\left(\eta_{1}\right)-\sum_{i=1}^{4} c_{i+4+8} \cdot f_{i}\left(\eta_{1}\right)=0
\end{array}
$$

Assuming that the angular displacement on the second contact point $\left(\eta_{2}\right)$ is 0 , the axial displacements, tangential directions and bending moments on the section point can be obtained with Eq. (21), namely

$$
\begin{gathered}
\left.\mu\right|_{\eta=\eta_{2}}=\left.1 \quad v\right|_{\eta=\eta_{2}}=0 \\
\left.\frac{d \mu}{d \eta}\right|_{\eta=\eta_{2}}=\left.0 \quad \frac{d v}{d \eta}\right|_{\eta=\eta_{2}}=\kappa
\end{gathered}
$$




$$
\left.\frac{d^{2} \mu}{d \eta^{2}}\right|_{\eta=\eta_{2}}=-\left.\kappa^{2} \quad \frac{d^{2} v}{d \eta^{2}}\right|_{\eta=\eta_{2}}=0
$$

Substituting Eq. (23) into the condition (3), namely Eq. (30)-(32), we obtain

$$
\begin{array}{cc}
\sum_{i=1}^{4} c_{i+8} \cdot d_{i}\left(\eta_{2}\right)=1 & \sum_{i=1}^{4} c_{i+4+8} \cdot d_{i}\left(\eta_{2}\right)=0 \\
\sum_{i=1}^{4} c_{i+8} \cdot e_{i}\left(\eta_{2}\right)=0 & \sum_{i=1}^{4} c_{i+4+8} \cdot e_{i}\left(\eta_{2}\right)=\kappa \\
\sum_{i=1}^{4} c_{i+8} \cdot f_{i}\left(\eta_{2}\right)=-\kappa^{2} & \sum_{i=1}^{4} c_{i+4+8} \cdot f_{i}\left(\eta_{2}\right)=0
\end{array}
$$

According to Lubinski's (1962) analysis, the parameter $\kappa$ is just equal to 1 . Here, we assume $\kappa=1$ is satisfied on the continuous contact section.

Substituting Eq. (23) into the condition (4), we obtain

$$
\sum_{i=1}^{4} c_{i+8} \cdot e_{i}\left(\eta_{1}\right) \cdot \cos \theta_{1}+\sum_{i=1}^{4} c_{i+4+8} \cdot e_{i}\left(\eta_{1}\right) \cdot \sin \theta_{1}=0
$$

From the above analyses, there are 19 unknown parameters including $c_{1}-c_{16}, \eta_{1}, \eta_{2}$ and $\theta_{1}$, and there are also corresponding 19 known equations including Eq. (24)-(29) and (33)-(36). To simplify the calculation, Eq. (24)-(29) and (33)-(34) are combined into a system of 16 linear equations with respect to $c_{1}-c_{16}$, and then $c_{1}-c_{16}$ expressed as the functions of $\eta_{1}, \eta_{2}$ and $\theta_{1}$ are obtained by solving the linear equations. Further substituting $c_{1}-c_{16}$ into Eq. (35) and (36), three nonlinear equations with respect to $\eta_{1}, \eta_{2}$ and $\theta_{1}$ are obtained. The nonlinear equations can be solved with Newton iteration method and the solutions are expressed as follows:

$$
\eta_{1}=1.696 \quad \eta_{2}=3.349 \quad \theta_{1}=-1.524
$$

At the critical transition point between the 3D lateral buckling and continuous contact buckling, the continuous contact length is equal to 0 , namely $\eta_{2}=\frac{\eta_{L}}{2}$. Therefore, the critical continuous contact buckling load is expressed by $\eta_{L, c o n}=6.698$ or

$$
F_{c o n}=\frac{9.09 \pi^{2} E I}{L^{2}}
$$

If the term $\kappa$ is taken to be 0 under the case of $\eta_{2}=\frac{\eta_{L}}{2}$, the solutions are obtained as follows:

$$
\eta_{1}=\frac{\pi}{\sqrt{2}} \quad \eta_{2}=\frac{\pi}{\sqrt{2}} \quad \theta_{1}=0
$$

From Eq. (39) we obtain $\eta_{L}=\sqrt{2} \pi$ which is just the critical 3D lateral buckling load. Therefore, the transition from the critical 3D lateral buckling to critical continuous contact buckling can be taken as the change of $\kappa$ from 0 to 1 in Eq. (33)-(35). The deflection curves of the tubular string at the 3D lateral buckling stage can be obtained by following the steps below:

(1) Set the term $\kappa$ equal to a certain value on the interval $[0,1]$.

(2) Solve the three terms including $\eta_{1}, \eta_{2}$ and $\theta_{1}$ from three nonlinear equations.

(3) Calculate the axial force $\left(\eta_{L}\right)$ with $\eta_{L}=2 \cdot \eta_{2}$.

(4) Calculate the values of $c_{1}-c_{16}$ with $\eta_{1}, \eta_{2}$ and $\theta_{1}$, and substitute them in Eq. (8).

At the critical condition of helical buckling, one pitch of helix is in continuous contact with the wellbore, namely 
$\eta_{2}=\frac{\eta_{L}-2 \pi}{2}$. Therefore, the critical helical buckling load is expressed by $\eta_{L, h e l}=12.981$ or

$$
F_{\text {hel }}=\frac{34.15 \pi^{2} E I}{L^{2}}
$$

The deflection curves of the tubular string at the continuous contact buckling and helical buckling stages can be obtained by following the steps below:

(1) Set the term $\kappa$ equal to 1.

(2) Calculate the values of $c_{1}-c_{16}$ with $\eta_{1}, \eta_{2}$ and $\theta_{1}$ in Eq. (37), and substitute them in Eq. (8).

(3) Calculate the length of the continuous contact section with $\eta_{L}-2 \eta_{2}$.

\subsection{Results and discussions}

\section{(1) Critical buckling loads}

The four critical buckling loads, namely Eq. (15), (19), (38) and (40), are expressed as the functions of the bending stiffness and length of the tubular string. Table 2 and Table 3 show the critical buckling loads for several kinds of tubular strings of different diameters and lengths. The tubular string length can be seen as the axial distance between two adjacent stabilizers on the tubular string. For the drill pipe (DP) with stabilizers, the helical buckling is usually hard to achieve. However, the lateral buckling and continuous contact buckling cannot be avoided for large stabilizer distance under high axial force. To overcome this shortcoming, the heavy weight drill pipe (HWDP) and drill collar (DC) of large-diameter and high-thickness are more prone to inhibit buckling. However, the 2D lateral buckling is difficult to avoid for large stabilizer distance, even the HWDP and DC are adopted. Therefore, to prevent the occurrence of tubular string buckling, the tubular string design should be conducted from two aspects: stabilizer distribution along the tubular string and the diameter and thickness of the tubular string.

Table 2-Critical buckling loads for several kinds of tubular strings when $\mathrm{L}=30 \mathrm{~m}$

\begin{tabular}{ccccccccc}
\hline Type & OD & ID & L & $\begin{array}{c}\text { EI } \\
\left(\mathrm{N} \times \mathrm{m}^{2}\right)\end{array}$ & $\begin{array}{c}\mathrm{F}_{2 \mathrm{D}} \\
(\mathrm{kN})\end{array}$ & $\begin{array}{c}\mathrm{F}_{3 \mathrm{D}} \\
(\mathrm{kN})\end{array}$ & $\begin{array}{c}\mathrm{F}_{\text {con }} \\
(\mathrm{kN})\end{array}$ & $\begin{array}{c}\mathrm{F}_{\text {hel }} \\
(\mathrm{kN})\end{array}$ \\
\hline DP & 73 & 54.6 & 30 & $2.002 \mathrm{E}+05$ & 2.20 & 8.78 & 19.95 \\
DP & 127 & 108.6 & 30 & $1.242 \mathrm{E}+06$ & 13.62 & 54.47 & 123.79 & 465.07 \\
HWDP & 127 & 76.2 & 30 & $2.323 \mathrm{E}+06$ & 25.47 & 101.90 & 231.56 & 869.96 \\
DC & 127 & 57.2 & 30 & $2.559 \mathrm{E}+06$ & 28.06 & 112.25 & 255.10 & 958.36 \\
DC & 177.8 & 57.2 & 30 & $1.014 \mathrm{E}+07$ & 111.23 & 444.92 & 1011.08 & 3798.52 \\
\hline
\end{tabular}

Table 3-Critical buckling loads for several kinds of tubular strings when $\mathrm{L}=10 \mathrm{~m}$

\begin{tabular}{ccccccccc}
\hline Type & $\begin{array}{c}\text { OD } \\
(\mathrm{mm})\end{array}$ & $\begin{array}{c}\mathrm{ID} \\
(\mathrm{mm})\end{array}$ & $\begin{array}{c}\mathrm{L} \\
(\mathrm{m})\end{array}$ & $\begin{array}{c}\mathrm{EI} \\
\left(\mathrm{N} \times \mathrm{m}^{2}\right)\end{array}$ & $\begin{array}{c}\mathrm{F}_{2 \mathrm{D}} \\
(\mathrm{kN})\end{array}$ & $\begin{array}{c}\mathrm{F}_{3 \mathrm{D}} \\
(\mathrm{kN})\end{array}$ & $\begin{array}{c}\mathrm{F}_{\text {con }} \\
(\mathrm{kN})\end{array}$ & $\begin{array}{c}\mathrm{F}_{\text {hel }} \\
(\mathrm{kN})\end{array}$ \\
\hline DP & 73 & 54.6 & 10 & $2.002 \mathrm{E}+05$ & 19.76 & 79.02 & 179.58 & 674.66 \\
DP & 127 & 108.6 & 10 & $1.242 \mathrm{E}+06$ & 122.57 & 490.26 & 1114.13 & 4185.63 \\
HWDP & 127 & 76.2 & 10 & $2.323 \mathrm{E}+06$ & 229.27 & 917.09 & 2084.08 & 7829.61 \\
DC & 127 & 57.2 & 10 & $2.559 \mathrm{E}+06$ & 252.57 & 1010.28 & 2295.86 & 8625.26 \\
DC & 177.8 & 57.2 & 10 & $1.014 \mathrm{E}+07$ & 1001.07 & 4004.29 & 9099.76 & 34186.65 \\
\hline
\end{tabular}




\section{(2) Contact states and deflection curves}

From the above analyses, the critical buckling loads between different buckling modes and the buckling behaviors under different buckling modes are determined. Fig. 3 shows the contact states of the top half portion of the entire tubular string under different axial forces. Here, the dimensionless axial distance is defined by

$$
\zeta=\frac{\eta}{\eta_{L}}=\frac{z}{L}
$$

Fig. 4 shows the deflection curves of the top half portion under different critical buckling loads, where the dimensionless radial displacement and angular displacement are defined by:

$$
\hat{r}=\sqrt{\mu^{2}+v^{2}} \quad \theta=\arctan \frac{v}{\mu}
$$

When the axial force is smaller than the critical load $F_{2 D}$, the tubular string remains straight and does not touch the wellbore. After the axial force reaches $F_{2 D}$, the tubular string buckles into a two-dimensional curve and the middle of the tubular string is in point contact with the wellbore shown in Fig. 3 and Fig. 4. At the 2D lateral buckling stage, the lateral displacement increases with the axial force. When the axial force achieves the critical load $F_{3 D}$, the two-dimensional configuration becomes unstable and the tubular string starts to buckle in the three-dimensional space. Meanwhile, the first contact point deviates from the middle point (the second contact point) and the top half portion of the entire tubular string includes two suspended sections, namely the first and second suspended sections shown in Fig. 3 and Fig. 4. With the increase of axial force, the first suspended section becomes shorter while the second suspended section becomes longer. According to the symmetry of the deflection curve, the total tubular string has four suspended sections at the 3D lateral buckling stage.

At the critical load $F_{c o n}$, there are two points which are in point contact with the wellbore and the radial deflection curve of the second suspend section is rather close to the wellbore surface shown in Fig. $4 \mathrm{~b}$. When the axial force exceeds the critical load $F_{c o n}$, the second point starts to deviates from the middle point and a continuous contact section is generated. When the axial force is equal to the critical value $F_{\text {hel }}$, the continuous contact section is one pitch of helix shown in Fig. 4c. In the continuous contact buckling and lateral buckling stages, the first and second suspended sections become shorter and the continuous contact section become longer with the increase of axial force shown in Fig. 3 . According to the symmetry of the deflection curve, the entire tubular string has four suspended sections near two ends and one middle continuous contact section in these two buckling stages. 


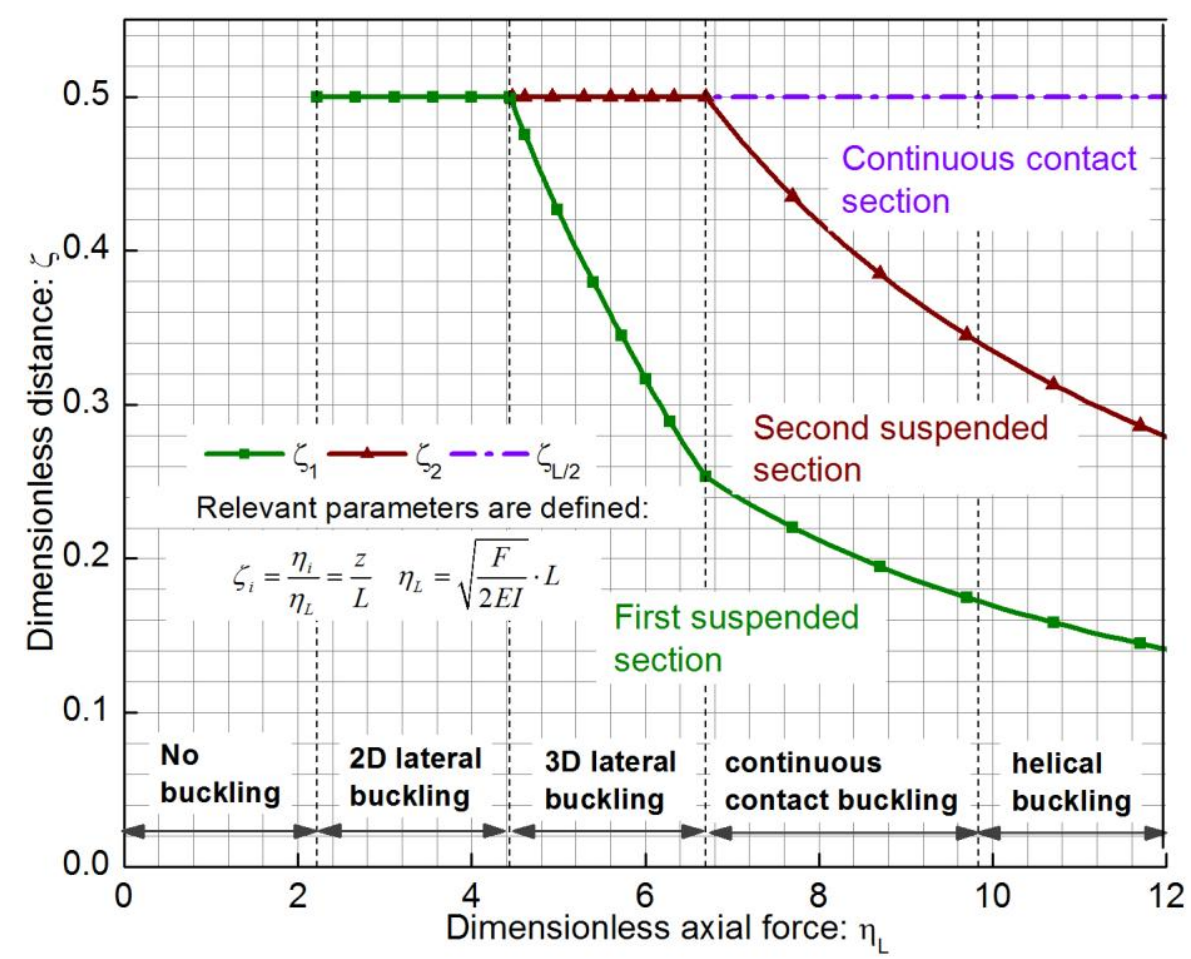

Fig. 3. The transition process of the contact states for the left half portion with the increase of axial force 


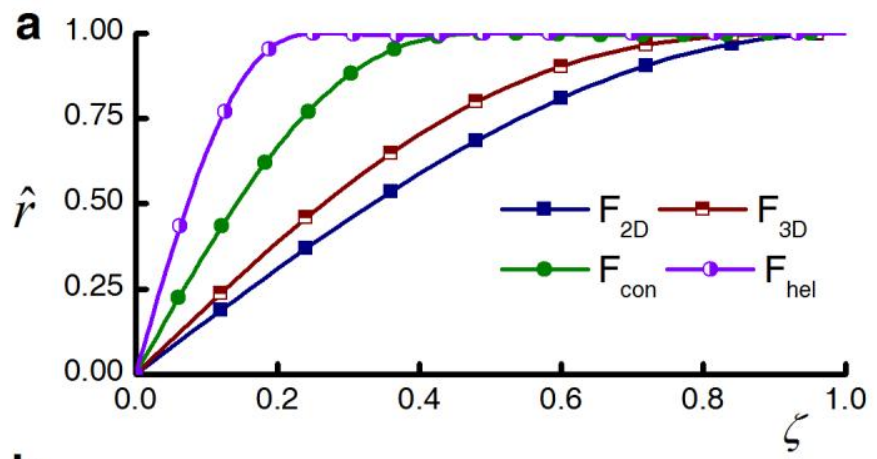

b
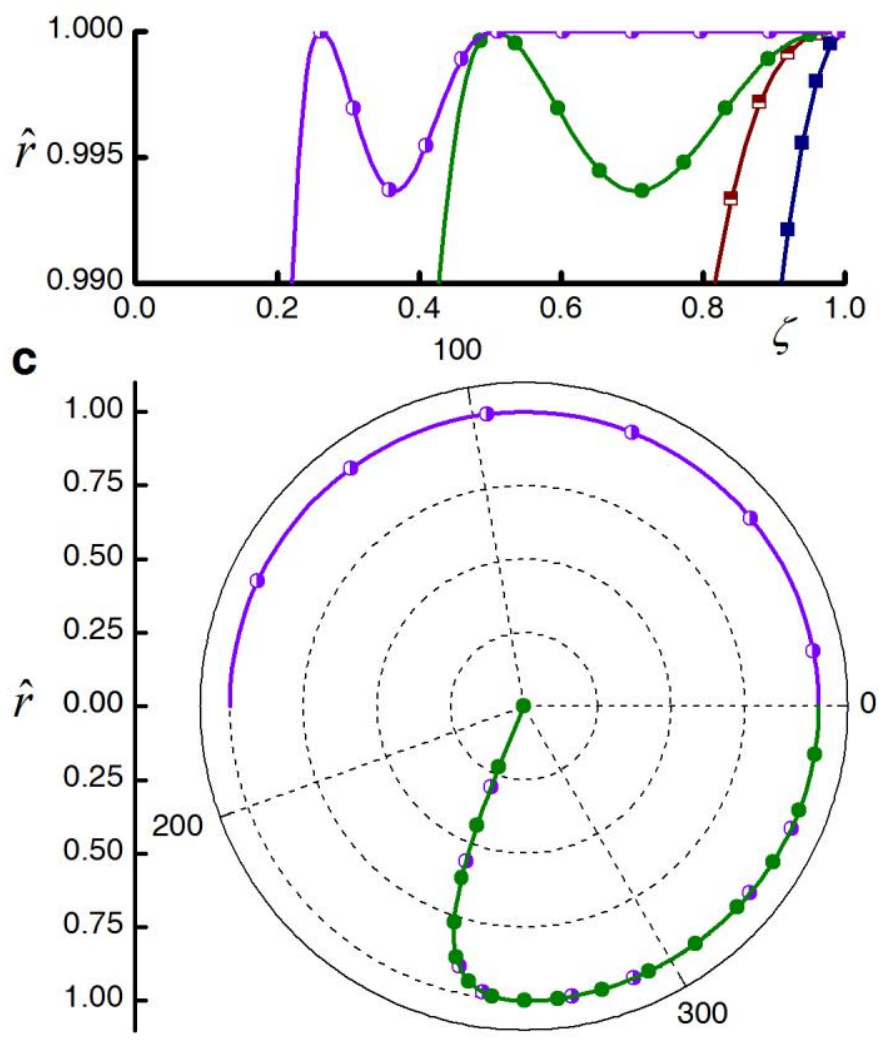

Fig. 4. The deflection curves of the left half portion under different critical buckling loads: (a) and (b) represent radial displacement vs. axial distance and (c) represents the radial displacement vs. angular displacement.

\section{Buckling model of tubular strings with weight}

\subsection{Model description}

For actual vertical wellbores, the effect of tubular string weight usually cannot be neglected. The existence of tubular string weight leads to the variation of axial force along the tubular string, which further complicates the tubular string buckling behaviors. In this section, we introduce the tubular string weight into the tubular string buckling model.

The buckling mode transition process for a tubular string with weight constrained in a vertical wellbore is similar to the weightless case. The entire process of the buckling mode transition is shown in Fig. 5. In the initial state (Fig. 5a), the tubular string keeps its initial non-buckling straight configuration. When weight on bit (WOB) exceeds a certain value, the straight configuration becomes unstable and the tubular string buckles into a curved configuration (Fig. 5b) in a 
two-dimensional (2D) plane. Different from the weightless tubular string case mentioned above, the 2D lateral buckling stage includes multiple sub-stages: first-order, second-order, third-order, etc. buckling. When the axial force reaches another critical value, the 2D lateral buckling state becomes unstable and the tubular string buckles in a 3D curved configuration (Fig. 5d). In the 3D lateral buckling stage, the tubular string touches the wellbore at several points distributed discretely. When the axial force exceeds the third critical value, the middle point contact is converted into the continuous contact between the middle portion of the tubular string and the wellbore (Fig. 5e). In the 3D continuous contact buckling stage, the entire tubular string is divided into four suspended sections and one continuous contact section. When the axial force reaches the fourth critical value, one pitch of helix is in continuous contact with the wellbore (Fig. 5f), and the critical axial force is taken as the critical helical buckling load.

Compared with the weightless case, the tubular string weight complicates the buckling problem from three aspects: (1) the symmetry of the tubular string deflection is broken; (2) the general solutions for the suspended and continuous contact sections become complicated: (3) the amount of calculation is greatly increased. In the following section, the critical loads between different buckling modes and the buckling configurations under different buckling modes are calculated. To achieve this objective, the same assumptions in the weightless tubular string case are adopted except that the tubular string weight is neglected.

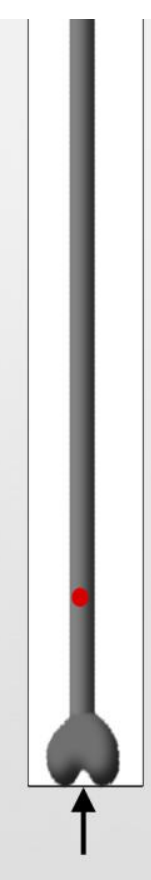

(a)

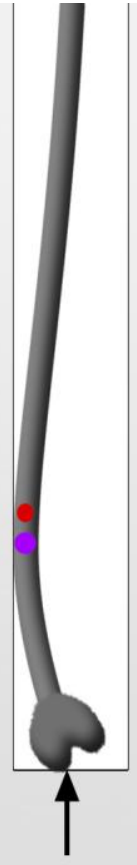

(b)

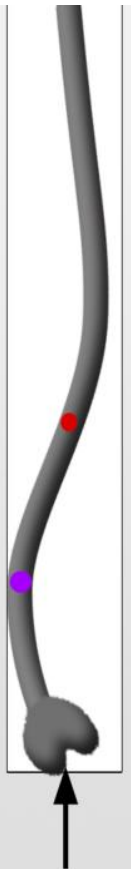

(c)

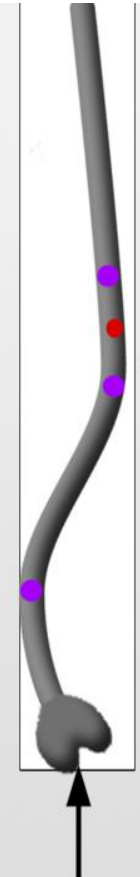

(d)

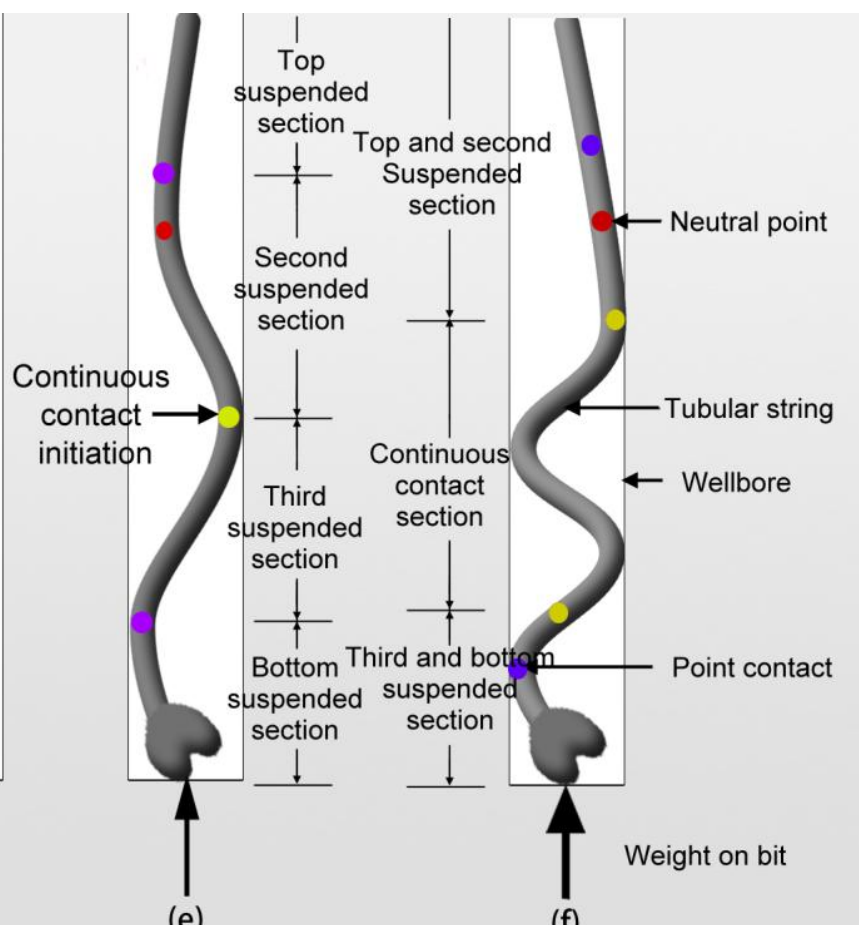

(e) (f)

Fig. 5. Buckling mode transition for a tubular string with weight constrained in a vertical wellbore: (a) initial straight configuration, (b) first-order 2D lateral buckling, (c) second-order 2D lateral buckling, (d) 3D lateral buckling, (e) continuous contact buckling and (f) helical buckling

\subsection{Non-buckling to 2D lateral buckling}

If the tubular string is in no contact or in contact with the inner surface of the wellbore at several single points in vertical wellbores, the governing equation of the tubular string deflection is expressed as

$$
E I \frac{d^{4} w}{d z^{4}}+\frac{d}{d z}\left(F \frac{d w}{d z}\right)=0
$$

Where $w$ is the lateral displacement along x-axis or y-axis, $z$ is the distance in the vertical direction. 
By introducing the dimensionless vertical distance $\eta=\left(\frac{q}{E I}\right)^{\frac{1}{3}} \cdot z$, dimensionless axial force $\xi=\left(\frac{q}{E I}\right)^{\frac{1}{3}} \cdot \frac{F}{q}$, dimensionless lateral displacement $\varsigma=\left(\frac{q}{E I}\right)^{\frac{1}{3}} \cdot w$, Eq. (43) is simplified into its dimensionless form:

$$
\frac{d^{4} \varsigma}{d \eta^{4}}+\frac{d}{d \eta}\left(\xi \frac{d \varsigma}{d \eta}\right)=0
$$

Further assuming that $z=0$ corresponds to the neutral point $(F=0)$, the axial force on the tubular string is equal to $F=q \cdot z$, where $q$ is weight per unit length of the tubular string. Thus, the dimensionless vertical distance $\eta$ is identical with the dimensionless axial force $\xi$, and Eq. (44) is simplified into

$$
\frac{d^{4} \varsigma}{d \eta^{4}}+\frac{d}{d \eta}\left(\eta \frac{d \varsigma}{d \eta}\right)=0
$$

The general solutions of Eq. (45) are expressed by:

$$
\varsigma=\sum_{i=1}^{4} c_{i} \cdot d_{i}(\eta)
$$

And the first, second and third derivatives of Eq. (46) with respect to $\eta$ are given below:

$$
\frac{d \varsigma}{d \eta}=\sum_{i=1}^{4} c_{i} \cdot e_{i}(\eta) \frac{d^{2} \varsigma}{d \eta^{2}}=\sum_{i=1}^{4} c_{i} \cdot f_{i}(\eta) \frac{d^{3} \varsigma}{d \eta^{3}}=\sum_{i=1}^{4} c_{i} \cdot g_{i}(\eta)
$$

Where $c_{i}$ are the integral constants and $d_{i}, e_{i}, f_{i}$ and $g_{i}$ are written in the form of power series with respect to $\eta$ :

$$
\begin{array}{lll}
d_{1}(\eta)=\sum_{n=0}^{\infty}(-1)^{n} \cdot \frac{\frac{1}{3 n+1} \eta^{3 n+1}}{\prod_{i=1}^{n}(3 i-1)(3 i-1+1)} \quad d_{2}(\eta)=\sum_{n=0}^{\infty}(-1)^{n} \cdot \frac{\frac{1}{3 n+2} \eta^{3 n+2}}{\prod_{i=1}^{n}(3 i-1+1)(3 i-1+2)} \quad d_{3}(\eta)=-\frac{1}{2} \sum_{n=0}^{\infty}(-1)^{n} \cdot \frac{\frac{1}{3 n+3} \eta^{3 n+3}}{\prod_{i=1}^{n}(3 i-1+2)(3 i-1+3)} \quad d_{4}(\eta)=1 \\
e_{1}(\eta)=\sum_{n=0}^{\infty}(-1)^{n} \cdot \frac{\eta^{3 n}}{\prod_{i=1}^{n}(3 i-1)(3 i-1+1)} \quad e_{2}(\eta)=\sum_{n=0}^{\infty}(-1)^{n} \cdot \frac{\eta^{3 n+1}}{\prod_{i=1}^{n}(3 i-1+1)(3 i-1+2)} \quad e_{3}(\eta)=-\frac{1}{2} \sum_{n=0}^{\infty}(-1)^{n} \cdot \frac{\eta^{3 n+2}}{\prod_{i=1}^{n}(3 i-1+2)(3 i-1+3)} \quad e_{4}(\eta)=0 \\
f_{1}(\eta)=\sum_{n=0}^{\infty}(-1)^{n} \cdot \frac{3 n \cdot \eta^{3 n-1}}{\prod_{i=1}^{n}(3 i-1)(3 i-1+1)} \quad f_{2}(\eta)=\sum_{n=0}^{\infty}(-1)^{n} \cdot \frac{(3 n+1) \cdot \eta^{3 n}}{\prod_{i=1}^{n}(3 i-1+1)(3 i-1+2)} \quad f_{3}(\eta)=-\frac{1}{2} \sum_{n=0}^{\infty}(-1)^{n} \cdot \frac{(3 n+2) \cdot \eta^{3 n+1}}{\prod_{i=1}^{n}(3 i-1+2)(3 i-1+3)} \quad f_{4}(\eta)=0 \\
g_{1}(\eta)=\sum_{n=0}^{\infty}(-1)^{n} \cdot \frac{(3 n) \cdot(3 n-1) \cdot \eta^{3 n-2}}{\prod_{i=1}^{n}(3 i-1)(3 i-1+1)} & g_{2}(\eta)=\sum_{n=0}^{\infty}(-1)^{n} \cdot \frac{(3 n+1) \cdot(3 n) \cdot \eta^{3 n-1}}{\prod_{i=1}^{n}(3 i-1+1)(3 i-1+2)} \quad g_{3}(\eta)=-\frac{1}{2} \sum_{n=0}^{\infty}(-1)^{n} \cdot \frac{(3 n+2) \cdot(3 n+1) \cdot \eta^{3 n}}{\prod_{i=1}^{n}(3 i-1+2)(3 i-1+3)} \quad g_{4}(\eta)=0
\end{array}
$$

Eq. (48) is also expressed in the form of special functions given in Appendix A.

Here we let $\eta_{0}=\xi_{0}=\left(\frac{q}{E I}\right)^{\frac{1}{3}} \cdot \frac{W O H}{q}$ denote the dimensionless weight on hook (WOH) and $\eta_{5}=\xi_{5}=\left(\frac{q}{E I}\right)^{\frac{1}{3}} \cdot \frac{W O B}{q}$ denote the dimensionless weight on bit (WOB). The parameter $\eta_{0}$ and $\eta_{5}$ also represent the dimensionless distances of the top hook and bottom drill bit with the neutral point as the origin. 


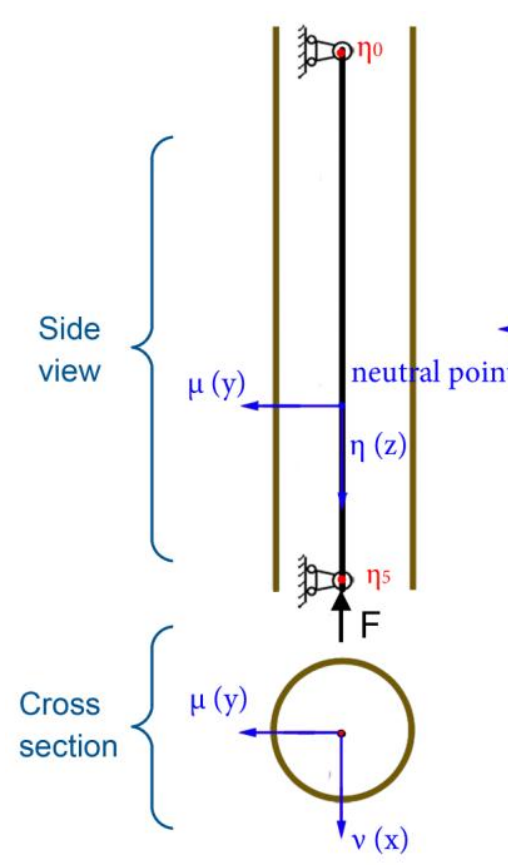

(a)

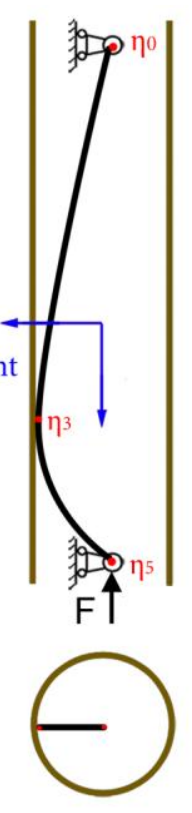

(b)

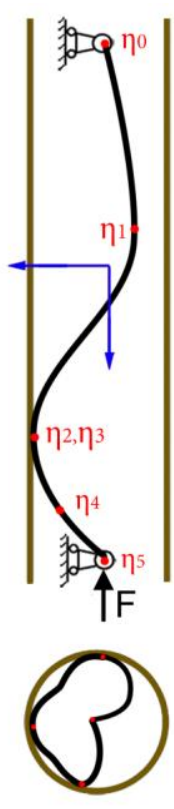

(c)

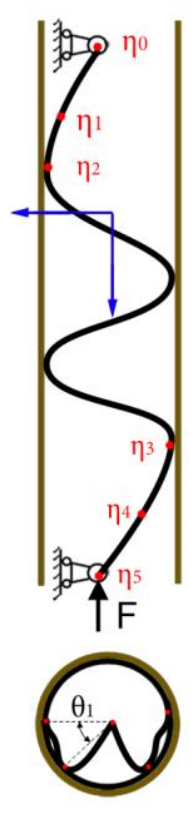

(d)

Fig. 6. Buckling configuration of a tubular string with weight constrained in a vertical wellbore

At the initial stage, the tubular string keeps its vertical line configuration shown in Fig. 6a. For the non-buckling to 2D lateral buckling case, the lateral deflection in the coordinate plane of $o-\eta-\mu$ is considered. The boundary conditions on the two ends are given as follows:

(1) The bending moments on the two ends $\left(\eta=\eta_{0}\right.$ and $\left.\eta=\eta_{5}\right)$ are both equal to 0 .

(2) The lateral displacements on the two ends $\left(\eta=\eta_{0}\right.$ and $\left.\eta=\eta_{5}\right)$ are both equal to 0 .

Similar to the weightless tubular string case, the critical 2D lateral buckling load is obtained when the following expression is satisfied:

$$
\left|\begin{array}{llll}
f_{1}\left(\eta_{0}\right) & f_{2}\left(\eta_{0}\right) & f_{3}\left(\eta_{0}\right) & f_{4}\left(\eta_{0}\right) \\
f_{1}\left(\eta_{5}\right) & f_{2}\left(\eta_{5}\right) & f_{3}\left(\eta_{5}\right) & f_{4}\left(\eta_{5}\right) \\
d_{1}\left(\eta_{0}\right) & d_{2}\left(\eta_{0}\right) & d_{3}\left(\eta_{0}\right) & d_{4}\left(\eta_{0}\right) \\
d_{1}\left(\eta_{5}\right) & d_{2}\left(\eta_{5}\right) & d_{3}\left(\eta_{5}\right) & d_{4}\left(\eta_{5}\right)
\end{array}\right|=0
$$

The critical WOBs $\left(\eta_{5}\right)$ under different WOHs $\left(\eta_{0}\right)$ are obtained by solving the above nonlinear equation with Newton iteration method.

After the WOB exceeds the first critical value, the tubular string buckles into a curved line of which the maximum lateral displacement is equal to the radial clearance between the tubular string and wellbore $r_{c}$ shown in Fig. 6b. Then the tubular string is divided into the two suspended sections, namely $\eta_{0}-\eta_{3}$ and $\eta_{3}-\eta_{5}$. The general solutions of tubular string deflection for the top and bottom suspended sections are respectively expressed by

$$
\mu_{1}=\sum_{i=1}^{4} c_{i} \cdot d_{i}(\eta) \quad \mu_{2}=\sum_{i=1}^{4} c_{i+4} \cdot d_{i}(\eta)
$$

The boundary conditions on the two ends and middle contact point are given as follows:

(1) The bending moments on the two ends $\left(\eta=\eta_{0}\right.$ and $\left.\eta=\eta_{5}\right)$ are both equal to 0 .

(2) The lateral displacements on the two ends $\left(\eta=\eta_{0}\right.$ and $\left.\eta=\eta_{5}\right)$ are both equal to 0 .

(3) The lateral displacement on the middle contact point $\left(\eta=\eta_{3}\right)$ is equal to the dimensionless radial clearance $\delta_{c}$, 
where $\delta_{c}$ is calculated by $\left(\frac{q}{E I}\right)^{\frac{1}{3}} \cdot r_{c}$.

(4) The deflection of the tubular string is tangential to the inner surface of the wellbore on the middle contact point $\left(\eta=\eta_{3}\right)$.

(5) The bending moment is continuous on the middle contact point $\left(\eta=\eta_{3}\right)$ between the two suspended sections.

According to the condition (1), we obtain:

$$
\sum_{i=1}^{4} c_{i} \cdot f_{i}\left(\eta_{0}\right)=0 \quad \sum_{i=1}^{4} c_{i+4} \cdot f_{i}\left(\eta_{5}\right)=0
$$

According to the condition (2), we obtain:

$$
\sum_{i=1}^{4} c_{i} \cdot d_{i}\left(\eta_{0}\right)=0 \quad \sum_{i=1}^{4} c_{i+4} \cdot d_{i}\left(\eta_{5}\right)=0
$$

According to the condition (3), we obtain:

$$
\sum_{i=1}^{4} c_{i} \cdot d_{i}\left(\eta_{3}\right)=\delta_{c} \quad \sum_{i=1}^{4} c_{i+4} \cdot d_{i}\left(\eta_{3}\right)=\delta_{c}
$$

According to the condition (4), we obtain:

$$
\sum_{i=1}^{4} c_{i} \cdot e_{i}\left(\eta_{3}\right)=0 \quad \sum_{i=1}^{4} c_{i+4} \cdot e_{i}\left(\eta_{3}\right)=0
$$

According to the condition (5), we obtain:

$$
\sum_{i=1}^{4} c_{i} \cdot f_{i}\left(\eta_{3}\right)-\sum_{i=1}^{4} c_{i+4} \cdot f_{i}\left(\eta_{3}\right)=0
$$

The combination of Eq. (51)-(54) can be expressed as 8 linear equations with respect to $c_{1}-c_{8}$. Substituting $c_{1}-c_{8}$ into Eq. (55), a nonlinear equation with $\eta_{0}, \eta_{3}$ and $\eta_{5}$ as variables is obtained. Usually, the WOH $\left(\eta_{0}\right)$ and WOB $\left(\eta_{5}\right)$ are given in advance, so the value of $\eta_{3}$ can be determined by solving the nonlinear equation. Substituting $c_{1}-c_{8}$ into Eq. (50), the deflection curve of the tubular string is determined. Note that the radial clearance $\delta_{c}$ has no effect on the critical buckling loads and contact point positions.

According to Lubinski's (1950) analysis, the tubular string may enter the second buckling mode in the 2D plane, which corresponds to the tangential direction of the tubular string on the top hook is just vertical:

$$
\sum_{i=1}^{4} c_{i} \cdot e_{i}\left(\eta_{0}\right)=0
$$

Substituting $c_{1}-c_{8}$ into Eq. (55) and (56), two nonlinear equations with respect to $\eta_{3}$ and $\eta_{5}$ are obtained. Solving the two nonlinear equations with Newton iteration method, the critical loads for second-order 2D lateral buckling $\left(\eta_{5}\right)$ under different values of hook load $\left(\eta_{0}\right)$ are determined.

\subsection{D lateral buckling to 3D lateral buckling}

The above analyses only consider the 2D lateral buckling on the coordinate plane o- $\eta-\mu$. However, when the axial force reaches a certain value, the 2D lateral buckling becomes unstable and the buckling also occurs on the coordinate plane o- $\eta-v$. The general solutions of tubular string deflection for the top and bottom suspended sections are respectively expressed by

$$
v_{1}=\sum_{i=1}^{4} c_{i+8} \cdot d_{i}(\eta) \quad v_{2}=\sum_{i=1}^{4} c_{i+4+8} \cdot d_{i}(\eta)
$$

At the 2D lateral buckling stage, the following conditions on the coordinate plane $o-\eta-v$ are satisfied:

(1) The bending moments on the two ends $\left(\eta=\eta_{0}\right.$ and $\left.\eta=\eta_{5}\right)$ are both equal to 0 .

(2) The lateral displacements on the two ends $\left(\eta=\eta_{0}\right.$ and $\left.\eta=\eta_{5}\right)$ are both equal to 0 .

(3) The lateral displacements on the middle contact point $\left(\eta=\eta_{3}\right)$ are equal to 0 and the first and second derivatives 
of the lateral displacements with respect to $\eta$ are continuous on the middle point between two suspended sections.

According to the above conditions, we obtain the following equations:

$$
\begin{aligned}
\sum_{i=1}^{4} c_{i+8} \cdot f_{i}\left(\eta_{0}\right)=0 & \sum_{i=1}^{4} c_{i+4+8} \cdot f_{i}\left(\eta_{5}\right)=0 \\
\sum_{i=1}^{4} c_{i} \cdot d_{i+8}\left(\eta_{0}\right)=0 & \sum_{i=1}^{4} c_{i+4+8} \cdot d_{i}\left(\eta_{5}\right)=0 \\
\sum_{i=1}^{4} c_{i+8} \cdot d_{i}\left(\eta_{3}\right)=0 & \sum_{i=1}^{4} c_{i+4+8} \cdot d_{i}\left(\eta_{3}\right)=0 \\
\sum_{i=1}^{4} c_{i+8} \cdot e_{i}\left(\eta_{3}\right)-\sum_{i=1}^{4} c_{i+4+8} \cdot e_{i}\left(\eta_{3}\right)=0 & \sum_{i=1}^{4} c_{i+8} \cdot f_{i}\left(\eta_{3}\right)-\sum_{i=1}^{4} c_{i+4+8} \cdot f_{i}\left(\eta_{3}\right)=0
\end{aligned}
$$

Eq. (58)-(61) have the physical meanings only if the determinant of the coefficient matrix of linear equations with respect to $c_{9}-c_{16}$ is equal to 0 , namely

$$
\left|\begin{array}{cccccccc}
f_{1}\left(\eta_{0}\right) & f_{2}\left(\eta_{0}\right) & f_{3}\left(\eta_{0}\right) & f_{4}\left(\eta_{0}\right) & 0 & 0 & 0 & 0 \\
0 & 0 & 0 & 0 & f_{1}\left(\eta_{5}\right) & f_{2}\left(\eta_{5}\right) & f_{3}\left(\eta_{5}\right) & f_{4}\left(\eta_{5}\right) \\
d_{1}\left(\eta_{0}\right) & d_{2}\left(\eta_{0}\right) & d_{3}\left(\eta_{0}\right) & d_{4}\left(\eta_{0}\right) & 0 & 0 & 0 & 0 \\
0 & 0 & 0 & 0 & d_{1}\left(\eta_{5}\right) & d_{2}\left(\eta_{5}\right) & d_{3}\left(\eta_{5}\right) & d_{4}\left(\eta_{5}\right) \\
d_{1}\left(\eta_{3}\right) & d_{2}\left(\eta_{3}\right) & d_{3}\left(\eta_{3}\right) & d_{4}\left(\eta_{3}\right) & 0 & 0 & 0 & 0 \\
0 & 0 & 0 & 0 & d_{1}\left(\eta_{3}\right) & d_{2}\left(\eta_{3}\right) & d_{3}\left(\eta_{3}\right) & d_{4}\left(\eta_{3}\right) \\
e_{1}\left(\eta_{3}\right) & e_{2}\left(\eta_{3}\right) & e_{3}\left(\eta_{3}\right) & e_{4}\left(\eta_{3}\right) & -e_{1}\left(\eta_{3}\right) & -e_{2}\left(\eta_{3}\right) & -e_{3}\left(\eta_{3}\right) & -e_{4}\left(\eta_{3}\right) \\
f_{1}\left(\eta_{3}\right) & f_{2}\left(\eta_{3}\right) & f_{3}\left(\eta_{3}\right) & f_{4}\left(\eta_{3}\right) & -f_{1}\left(\eta_{3}\right) & -f_{2}\left(\eta_{3}\right) & -f_{3}\left(\eta_{3}\right) & -f_{4}\left(\eta_{3}\right)
\end{array}\right|=0
$$

Note that the above equation cannot be solved directly due to the existence of two undetermined variables $\eta_{3}$ and $\eta_{5}$ except $\eta_{0}$. In order to ensure the integrity of problem, another nonlinear equation with $\eta_{3}$ and $\eta_{5}$ as variables is obtained by substituting $c_{1}-c_{8}$ into Eq. (55) in the coordinate plane o- $\eta-\mu$. As a result, the values of $\eta_{3}$ and $\eta_{5}$ for the critical 3D lateral buckling are determined by solving the two nonlinear equations with Newton iteration method.

\subsection{D lateral buckling, continuous contact buckling and helical buckling}

With the WOB further exceeding the critical 3D lateral buckling load, the tubular string becomes 3D lateral buckling, continuous contact buckling or even helical buckling. In the 3D lateral buckling stage, the entire tubular string is depicted by four sections: top, second, third and bottom suspended sections. If the tubular string enters into the continuous contact buckling and helical buckling stages, the middle portion of the tubular string becomes in continuous contact with the wellbore.

\section{(a) Continuous contact section}

The buckling differential equation for depicting the continuous contact section in vertical wells is expressed by (Mitchell, 1988)

$$
\frac{d^{4} \theta}{d z^{4}}-6\left(\frac{d \theta}{d z}\right)^{2} \frac{d^{2} \theta}{d z^{2}}+3 \frac{M_{T}}{E I} \frac{d \theta}{d z} \frac{d^{2} \theta}{d z^{2}}+\frac{d}{d z}\left(\frac{F}{E I} \frac{d \theta}{d z}\right)=0
$$

By introducing the dimensionless vertical distance $\eta=\left(\frac{q}{E I}\right)^{\frac{1}{3}} \cdot z$, dimensionless axial compression $\xi=\left(\frac{q}{E I}\right)^{\frac{1}{3}} \cdot \frac{F}{q}$, and dimensionless torque $m=\left(\frac{q}{E I}\right)^{\frac{2}{3}} \cdot \frac{M_{T}}{q}$, the above equation is transformed into its dimensionless form: 


$$
\frac{d^{4} \theta}{d \eta^{4}}-6\left(\frac{d \theta}{d \eta}\right)^{2} \frac{d^{2} \theta}{d \eta^{2}}+3 m \frac{d \theta}{d \eta} \frac{d^{2} \theta}{d \eta^{2}}+\frac{d}{d \eta}\left(\xi \frac{d \theta}{d \eta}\right)=0
$$

The integration of Eq. (64) with respect to the variable $\eta$ produces:

$$
\frac{d^{3} \theta}{d \eta^{3}}-2\left(\frac{d \theta}{d \eta}\right)^{3}+\frac{3}{2} m\left(\frac{d \theta}{d \eta}\right)^{2}+\xi \frac{d \theta}{d \eta}=C
$$

Generally, the third derivative $\frac{d^{3} \theta}{d \eta^{3}}$ in the above equation is rather small with respect to the first derivative $\frac{d \theta}{d \eta}$. Huang et al. (2015) pointed out that $\mathrm{C}=0$ corresponds to the first kind of boundary condition, though Huang's model only considers the weightless tubular string case. Here, we simplify Eq. (65) by neglecting the term $\frac{d^{3} \theta}{d \eta^{3}}$ and letting $\mathrm{C}=0$. Thus, we obtain

$$
2\left(\frac{d \theta}{d \eta}\right)^{2}-\frac{3}{2} m\left(\frac{d \theta}{d \eta}\right)-\xi=0
$$

For the no torque case, we obtain

$$
\frac{d \theta}{d \eta}=\sqrt{\frac{\xi}{2}}
$$

If the friction force is neglected, the axial force is calculated by $F=q \cdot z$. Therefore, the dimensionless axial force $\xi$ is identical with the dimensionless vertical distance $\eta$, namely

$$
\xi=\eta
$$

and Eq. (67) is simplified into

$$
\frac{d \theta}{d \eta}=\sqrt{\frac{\eta}{2}}
$$

The angular displacement of the continuous contact section is the integral of Eq. (69),

$$
\theta=\frac{\sqrt{2}}{3} \eta^{\frac{3}{2}}+c
$$

Where $c$ is the integral constant.

The lateral displacements of the continuous contact section can be expressed as the functions of $\theta$ :

$$
\mu_{3}=\delta_{c} \cdot \cos \theta \quad v_{3}=\delta_{c} \cdot \sin \theta
$$

By assuming that the angular displacement $\theta$ is equal to 0 at the contact point $\eta_{3}$, the lateral displacements and the first and second derivatives of the lateral displacements respect to $\eta$ are calculated by

$$
\begin{gathered}
\mu_{3}=\delta_{c} \quad v_{3}=0 \\
\frac{d \mu_{3}}{d \eta}=0 \quad \frac{d v_{3}}{d \eta}=\sqrt{\frac{\eta}{2}} \cdot \delta_{c} \\
\frac{d^{2} \mu_{3}}{d \eta^{2}}=-\frac{\eta}{2} \cdot \delta_{c} \quad \frac{d^{2} v_{3}}{d \eta^{2}}=\frac{1}{2 \sqrt{2 \eta}} \cdot \delta_{c}
\end{gathered}
$$

\section{(b) Top and second suspended sections}

The top and second suspended sections above the continuous contact section are depicted by beam-column models shown in Fig. 5 and Fig. 6d, in which the angular displacement of the connecting point between these two suspended beams is assumed to be $\theta_{1}$. In this case, the following conditions are satisfied:

(1) The lateral displacements and bending moments on the top end $\left(\eta=\eta_{0}\right)$ are both equal to 0 . 
(2) The lateral displacements, tangential directions and bending moments are continuous between the top and second suspended sections $\left(\eta=\eta_{1}\right)$.

(3) The deflection curve of the tubular string on the connecting point $\left(\eta=\eta_{1}\right)$ is tangent to the inner surface of the wellbore.

(4) The lateral displacements, tangential directions and bending moments are continuous between the second suspended section and continuous contact section $\left(\eta=\eta_{2}\right)$.

The general solutions of the top and second suspended sections are expressed by

$$
\begin{array}{ll}
\mu_{1}=\sum_{i=1}^{4} c_{i} \cdot d_{i}(\eta) & \mu_{2}=\sum_{i=1}^{4} c_{i+4} \cdot d_{i}(\eta) \\
v_{1}=\sum_{i=1}^{4} c_{i+8} \cdot d_{i}(\eta) & v_{2}=\sum_{i=1}^{4} c_{i+4+8} \cdot d_{i}(\eta)
\end{array}
$$

According to the condition (1), we obtain

$$
\begin{array}{ll}
\sum_{i=1}^{4} c_{i} \cdot d_{i}\left(\eta_{0}\right)=0 & \sum_{i=1}^{4} c_{i+8} \cdot d_{i}\left(\eta_{0}\right)=0 \\
\sum_{i=1}^{4} c_{i} \cdot f_{i}\left(\eta_{0}\right)=0 & \sum_{i=1}^{4} c_{i+8} \cdot f_{i}\left(\eta_{0}\right)=0
\end{array}
$$

According to the condition (2), we obtain

$$
\begin{array}{cl}
\sum_{i=1}^{4} c_{i} \cdot d_{i}\left(\eta_{1}\right)=\delta_{c} \cos \theta_{1} & \sum_{i=1}^{4} c_{i+8} \cdot d_{i}\left(\eta_{1}\right)=\delta_{c} \sin \theta_{1} \\
\sum_{i=1}^{4} c_{i+4} \cdot d_{i}\left(\eta_{1}\right)=\delta_{c} \cos \theta_{1} & \sum_{i=1}^{4} c_{i+4+8} \cdot d_{i}\left(\eta_{1}\right)=\delta_{c} \sin \theta_{1} \\
\sum_{i=1}^{4} c_{i} \cdot e_{i}\left(\eta_{1}\right)-\sum_{i=1}^{4} c_{i+4} \cdot e_{i}\left(\eta_{1}\right)=0 & \sum_{i=1}^{4} c_{i+8} \cdot e_{i}\left(\eta_{1}\right)-\sum_{i=1}^{4} c_{i+4+8} \cdot e_{i}\left(\eta_{1}\right)=0 \\
\sum_{i=1}^{4} c_{i} \cdot f_{i}\left(\eta_{1}\right)-\sum_{i=1}^{4} c_{i+4} \cdot f_{i}\left(\eta_{1}\right)=0 & \sum_{i=1}^{4} c_{i+8} \cdot f_{i}\left(\eta_{1}\right)-\sum_{i=1}^{4} c_{i+4+8} \cdot f_{i}\left(\eta_{1}\right)=0
\end{array}
$$

According to condition (3), we obtain

$$
\sum_{i=1}^{4} c_{i} \cdot e_{i}\left(\eta_{1}\right) \cdot \cos \theta_{1}+\sum_{i=1}^{4} c_{i+8} \cdot e_{i}\left(\eta_{1}\right) \cdot \sin \theta_{1}=0
$$

According to condition (4), we obtain

$$
\begin{gathered}
\sum_{i=1}^{4} c_{i+4} \cdot d_{i}\left(\eta_{2}\right)=1 \sum_{i=1}^{4} c_{i+4+8} \cdot d_{i}\left(\eta_{2}\right)=0 \\
\sum_{i=1}^{4} c_{i+4} \cdot e_{i}\left(\eta_{2}\right)=0 \quad \sum_{i=1}^{4} c_{i+4+8} \cdot e_{i}\left(\eta_{2}\right)=\sqrt{\frac{\eta_{2}}{2}} \cdot \delta_{c} \\
\sum_{i=1}^{4} c_{i+4} \cdot f_{i}\left(\eta_{2}\right)=-\frac{\eta_{2}}{2} \cdot \delta_{c} \quad \sum_{i=1}^{4} c_{i+4+8} \cdot f_{i}\left(\eta_{2}\right)=\frac{1}{2 \sqrt{2 \eta_{2}}} \cdot \delta_{c}
\end{gathered}
$$

The combination of Eq. (76)-(81) and (83)-(84) produces 16 linear equations with respect to $c_{1}-c_{16}$. Solving $c_{1}-c_{16}$ from the linear equations and substituting them into Eq. (82) and (85), we obtain 3 nonlinear equations with respect to $\eta_{0}, \eta_{1}, \eta_{2}$ and $\theta_{1}$. Generally, the $\mathrm{WOH}\left(\eta_{0}\right)$ is given in advance, and the other parameters can be determined by solving the nonlinear equations with Newton iteration method. Note that the radial clearance $\delta_{c}$ has no effect on the critical buckling loads and contact point positions.

\section{(c) Third and bottom suspended sections}

Similar to the top and second suspended sections, the third and bottom suspended sections below the continuous contact section are also depicted by beam-column models shown in Fig. 5 and Fig. 6d, in which the angular displacement 
of the connecting point between these two suspended beams is assumed to be $\theta_{4}$. In this case, the following conditions are satisfied:

(1) The lateral displacements and bending moments on the bottom end $\left(\eta=\eta_{5}\right)$ are both equal to 0 .

(2) The lateral displacements, tangential directions and bending moments are continuous between the third and bottom suspended sections $\left(\eta=\eta_{4}\right)$.

(3) The deflection of the tubular string on the connecting point $\left(\eta=\eta_{4}\right)$ is tangent to the inner surface of the wellbore.

(4) The lateral displacements, tangential directions and bending moments are continuous between the third suspended section and continuous contact section $\left(\eta=\eta_{3}\right)$.

The general solutions of the third and bottom suspended sections are expressed by

$$
\begin{aligned}
& \mu_{4}=\sum_{i=1}^{4} c_{i} \cdot d_{i}(\eta) \quad \mu_{5}=\sum_{i=1}^{4} c_{i+4} \cdot d_{i}(\eta) \\
& v_{4}=\sum_{i=1}^{4} c_{i+8} \cdot d_{i}(\eta)
\end{aligned}
$$

According to the condition (1), we obtain

$$
\begin{array}{ll}
\sum_{i=1}^{4} c_{i} \cdot d_{i}\left(\eta_{5}\right)=0 & \sum_{i=1}^{4} c_{i+8} \cdot d_{i}\left(\eta_{5}\right)=0 \\
\sum_{i=1}^{4} c_{i} \cdot f_{i}\left(\eta_{5}\right)=0 & \sum_{i=1}^{4} c_{i+8} \cdot f_{i}\left(\eta_{5}\right)=0
\end{array}
$$

According to the condition (2), we obtain

$$
\begin{aligned}
& \sum_{i=1}^{4} c_{i} \cdot d_{i}\left(\eta_{4}\right)=\delta_{c} \cos \theta_{4} \quad \sum_{i=1}^{4} c_{i+8} \cdot d_{i}\left(\eta_{4}\right)=\delta_{c} \sin \theta_{4} \\
& \sum_{i=1}^{4} c_{i+4} \cdot d_{i}\left(\eta_{4}\right)=\delta_{c} \cos \theta_{4} \quad \sum_{i=1}^{4} c_{i+4+8} \cdot d_{i}\left(\eta_{4}\right)=\delta_{c} \sin \theta_{4} \\
& \sum_{i=1}^{4} c_{i} \cdot e_{i}\left(\eta_{4}\right)-\sum_{i=1}^{4} c_{i+4} \cdot e_{i}\left(\eta_{4}\right)=0 \quad \sum_{i=1}^{4} c_{i+8} \cdot e_{i}\left(\eta_{4}\right)-\sum_{i=1}^{4} c_{i+4+8} \cdot e_{i}\left(\eta_{4}\right)=0 \\
& \sum_{i=1}^{4} c_{i} \cdot f_{i}\left(\eta_{4}\right)-\sum_{i=1}^{4} c_{i+4} \cdot f_{i}\left(\eta_{4}\right)=0 \quad \sum_{i=1}^{4} c_{i+8} \cdot f_{i}\left(\eta_{4}\right)-\sum_{i=1}^{4} c_{i+4+8} \cdot f_{i}\left(\eta_{4}\right)=0
\end{aligned}
$$

According to condition (3), we obtain

$$
\sum_{i=1}^{4} c_{i} \cdot e_{i}\left(\eta_{4}\right) \cdot \cos \theta_{4}+\sum_{i=1}^{4} c_{i+8} \cdot e_{i}\left(\eta_{4}\right) \cdot \sin \theta_{4}=0
$$

According to condition (4), we obtain

$$
\begin{gathered}
\sum_{i=1}^{4} c_{i+4} \cdot d_{i}\left(\eta_{3}\right)=1 \sum_{i=1}^{4} c_{i+4+8} \cdot d_{i}\left(\eta_{3}\right)=0 \\
\sum_{i=1}^{4} c_{i+4} \cdot e_{i}\left(\eta_{3}\right)=0 \quad \sum_{i=1}^{4} c_{i+4+8} \cdot e_{i}\left(\eta_{3}\right)=\sqrt{\frac{\eta_{3}}{2}} \cdot \delta_{c} \\
\sum_{i=1}^{4} c_{i+4} \cdot f_{i}\left(\eta_{3}\right)=-\frac{\eta_{3}}{2} \cdot \delta_{c} \quad \sum_{i=1}^{4} c_{i+4+8} \cdot f_{i}\left(\eta_{3}\right)=\frac{1}{2 \sqrt{2 \eta_{3}}} \cdot \delta_{c}
\end{gathered}
$$

Similar to the solutions of the top and second suspended sections, three nonlinear equations with respect to $\eta_{3}, \eta_{4}$, $\eta_{5}$ and $\theta_{4}$ are obtained. If one parameter is given such as $\eta_{5}$, the other three parameters can be determined by solving the nonlinear equations.

From the above analyses we can see that the tubular string deflection in top and first suspended, middle continuous contact, third and bottom suspended sections are all determined. The buckling configuration of the entire tubular string 
can be obtained by the following steps:

(1) If the WOH $\left(\eta_{0}\right)$ is given in advance, the top and second suspended sections $\left(\eta_{1}, \eta_{2}\right)$ are determined with the analysis in part (b).

(2) If the WOB $\left(\eta_{5}\right)$ is given in advance, the third and bottom suspended sections $\left(\eta_{3}, \eta_{4}\right)$ are determined with the analysis in part (c).

(3) If the expression $\eta_{2}>\eta_{3}$ is satisfied, the results is invalid; If the expression $\eta_{2}=\eta_{3}$ is satisfied, the critical continuous contact buckling load is obtained; If the expression $\eta_{2}<\eta_{3}$ is satisfied, the tubular string on the continuous section between $\eta_{2}$ and $\eta_{3}$ is depicted by the expression in part (a); If the angular displacement on the continuous contact is equal to one period of helix, namely $\frac{\sqrt{2}}{3} \eta_{3}^{\frac{3}{2}}-\frac{\sqrt{2}}{3} \eta_{2}^{\frac{3}{2}}=2 \pi$, the critical helical bucking load is obtained.

At the 3D lateral buckling stage, the entire tubular string includes four suspended sections depicted by beam-column models. Therefore, the 3D lateral buckling corresponds to the case of $\eta_{2}=\eta_{3}$ mentioned above. Meanwhile, the conditions on the middle contact point $\left(\eta=\eta_{2}, \eta_{3}\right)$ in Eq. (84) and (85) should be re-written as

$$
\begin{gathered}
\sum_{i=1}^{4} c_{i+4} \cdot e_{i}\left(\eta_{2}\right)=0 \quad \sum_{i=1}^{4} c_{i+4+8} \cdot e_{i}\left(\eta_{2}\right)=\kappa \cdot \sqrt{\frac{\eta_{2}}{2}} \cdot \delta_{c} \\
\sum_{i=1}^{4} c_{i+4} \cdot f_{i}\left(\eta_{2}\right)=-\frac{\eta_{3}{ }^{0}}{2} \cdot \delta_{c}+\left(-\frac{\eta_{2}}{2}+\frac{\eta_{3}{ }^{0}}{2}\right) \cdot \kappa \cdot \delta_{c} \quad \sum_{i=1}^{4} c_{i+4+8} \cdot f_{i}\left(\eta_{2}\right)=\frac{1}{2 \sqrt{2 \eta_{2}}} \cdot \kappa \cdot \delta_{c}
\end{gathered}
$$

Where $-\frac{\eta_{3}{ }^{0}}{2} \cdot \delta_{c}$ denotes the second derivative of the lateral displacement on the middle contact point $\left(\eta=\eta_{3}\right)$ at the critical 3D lateral buckling load. From Eq. (97)-(98) we can see that $\kappa=0$ represents the critical 3D lateral buckling condition and $\kappa=1$ represents the critical continuous contact buckling condition. Therefore, the transition from the critical 3D lateral buckling to critical continuous contact buckling can be taken as the change of $\kappa$ from 0 to 1.

\section{(d) Fitting formulae}

The above calculation procedures indicate that iteration method must be used to calculate the critical buckling loads $\left(\eta_{5}\right)$. Here, we adopt the following procedures:

(1) If the $\mathrm{WOH}\left(\eta_{0}\right)$ is given in advance, the top and second suspended sections $\left(\eta_{1}, \eta_{2}\right)$ are determined with the analysis in part (b).

(2) If the term $\left(\eta_{3}\right)$ is given in advance, the third and bottom suspended sections $\left(\eta_{4}, \eta_{5}\right)$ are determined with the analysis in part (c).

(3) If the expression $\eta_{2}=\eta_{3}$ is satisfied, the critical continuous contact buckling load is obtained; If the expression $\eta_{2}<\eta_{3}$ is satisfied, the tubular string on the continuous section between $\eta_{2}$ and $\eta_{3}$ is depicted by the expression in part (a); If the expression $\frac{\sqrt{2}}{3} \eta_{3}^{\frac{3}{2}}-\frac{\sqrt{2}}{3} \eta_{2}^{\frac{3}{2}}=2 \pi$ is satisfied, the critical helical bucking load is obtained.

If the parameter $\eta$ is a negative value of large absolute magnitude, the calculation of the general functions $\left(d_{i}(\eta)\right.$, $e_{i}(\eta), f_{i}(\eta)$ and $\left.g_{i}(\eta)\right)$ becomes unstable. Thus, the buckling transition model mentioned above is not feasible for the case of $\eta_{0} \rightarrow-\infty$. To overcome this shortcoming, the buckling configuration on the interval of $\eta_{0} \in[-8,0]$ is calculated given in Fig. 7 and the interval of $\eta_{0}$ is extended to $\eta_{0} \in[-\infty, 0]$ with the fitting formulae of the results. It is indicated that the fitting results on the top and second suspended sections given in Eq. (99)-(100) are in good agreement 
with the calculation results.

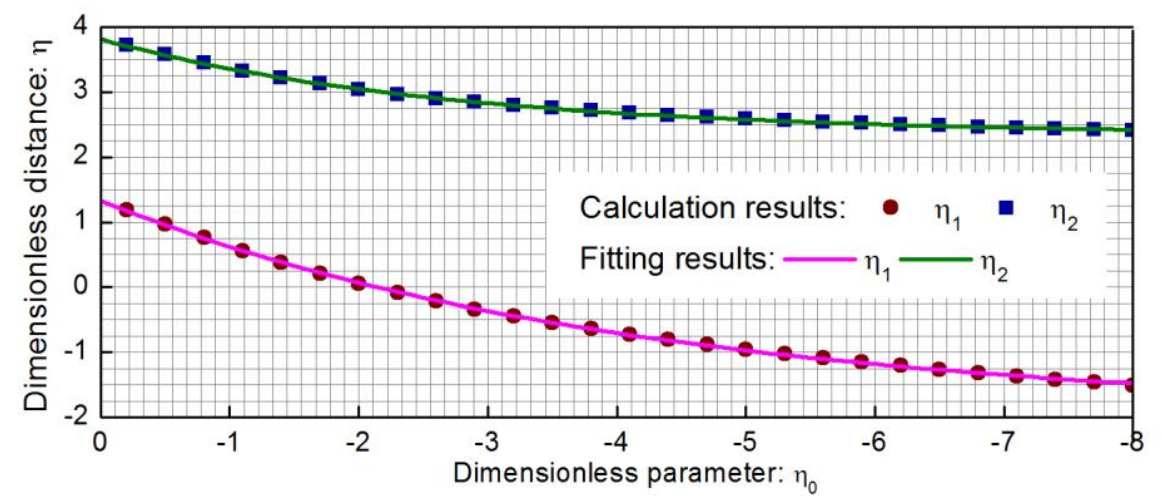

Fig. 7. Dimensionless distances of the top and second suspend sections.

$$
\begin{aligned}
& \eta_{1}=-1.9472+3.2755 e^{0.2421 \eta_{0}} \\
& \eta_{2}=2.3451+1.4669 e^{0.3707 \eta_{0}}
\end{aligned}
$$

Similarly, the buckling configuration on the interval of $\eta_{3} \in[2,11]$ is calculated in Fig. 8 and the fitting formulae of on the interval of $\eta_{3} \in[2,+\infty]$ are obtained. It is indicated that the fitting results on the third and bottom suspended sections given in Eq. (101) and (102) are in good consistence with the calculation results.

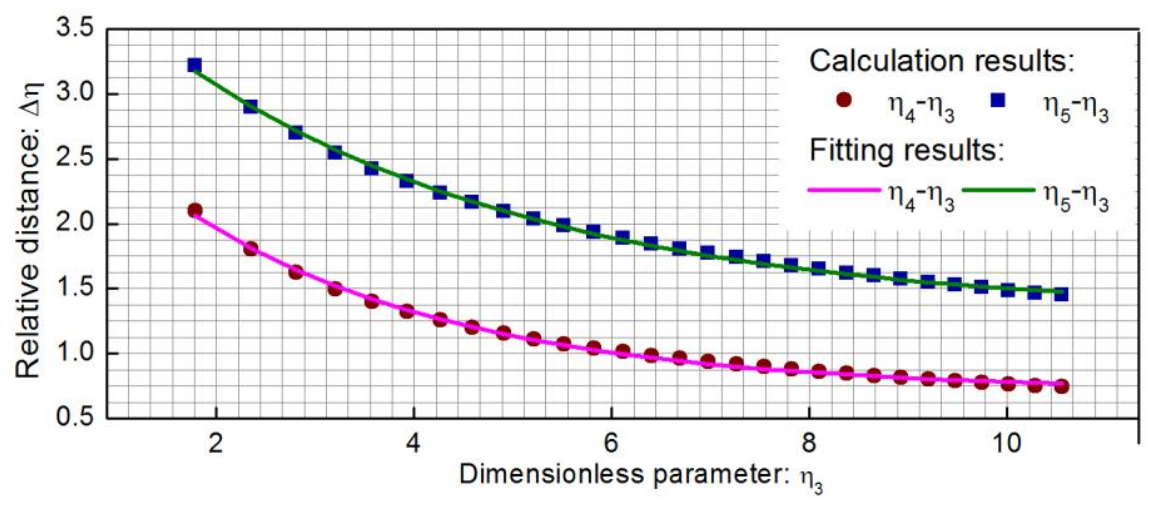

Fig. 8. Dimensionless distances of the third and bottom suspended sections.

$$
\begin{aligned}
& \eta_{4}=\eta_{3}+0.7131+2.5651 e^{-0.3602 \eta_{3}} \\
& \eta_{5}=\eta_{3}+1.3089+3.0432 e^{-0.2753 \eta_{3}}
\end{aligned}
$$

According to the fitting formulae (Eq. (99)-(102)), the critical conditions for the critical continuous contact buckling and helical buckling can be easily obtained by following the procedure (3):

\subsection{Results and discussions}

\section{(1) Critical buckling loads}

The critical bucking loads for buckling modes and the buckling solutions under different buckling modes have been 
calculation in the above analyses. The total bucking phase is divided into five cases, namely no buckling, 2D lateral buckling, 3D lateral buckling, continuous contact buckling and helical buckling by four critical loads, namely critical 2D lateral buckling, critical 3D lateral buckling, critical continuous contact buckling and critical helical buckling. These four critical WOBs are respectively denoted by $\eta_{5,2 D}, \eta_{5,3 D}, \eta_{5, \text { con }}$ and $\eta_{5, \text { hel }}$. The $2 \mathrm{D}$ lateral buckling is further divided into first, second, and third-order buckling modes, of which the critical loads are denoted by $\eta_{5,2 D, 1 t h}, \eta_{5,2 D, 2 t h}$ and $\eta_{5,2 D, 3 t h}$. For the case of $\eta_{0}<-1$, the second-order 2D lateral buckling mode is achieved before the critical 3D lateral buckling condition. However, the tubular string does not enter the second-order 2D lateral mode but buckles in the more stable 3D lateral mode under the case of $\eta_{0} \geq-1$. The third-order 2D lateral buckling cannot be achieved for the tubular string has been in the continuous contact buckling mode before reaching the third-order critical load. In the following analyses, only the first-order $2 \mathrm{D}$ lateral buckling is considered, so $\eta_{5,2 D}$ is identical with $\eta_{5,2 D, 1 \mathrm{th}}$.

For the convenience of application, the critical 2D and 3D lateral buckling loads are given in the form of fitting formulae,

$$
\begin{aligned}
& \eta_{5,2 D}=1.8546+0.7466 e^{0.3689 \eta_{0}} \\
& \eta_{5,3 D}=3.9494+0.4264 e^{0.2783 \eta_{0}}
\end{aligned}
$$

For the continuous contact buckling and helical buckling, the critical loads are obtained with Eq. (99)-(102), namely

$$
\begin{gathered}
\eta_{5, \text { con }}=2.3451+1.4669 e^{0.3707 \eta_{0}}+1.3089+3.0432 e^{-0.2753\left(2.3451+1.4669 e^{0.3707 \eta_{0}}\right)} \\
\eta_{5, \text { hel }}=\left[3 \sqrt{2} \pi+\left(2.3451+1.4669 e^{0.3707 \eta_{0}}\right)^{\frac{3}{2}}\right]^{\frac{2}{3}}+1.3089+3.0432 e^{-0.2753\left[3 \sqrt{2} \pi+\left(2.3451+1.4669 e^{0.37070}\right)^{\frac{3}{2}}\right]^{\frac{2}{3}}}
\end{gathered}
$$

All the critical buckling loads increase with the $\mathrm{WOH}\left(\eta_{0}\right)$, indicating that reducing the length of the tubular string above the neutral point can increase the critical buckling loads. When WOH is relatively small, for example $\eta_{0}<-6$, the critical buckling loads tend to stable values. It is roughly estimated that $\eta_{5,3 D}$ and $\eta_{5, c o n}$ are respectively two and three times of $\eta_{5,2 D}$, and $\eta_{5, h e l}$ is more than four times of $\eta_{5,2 D}$. To be specific, the upper limits of WOB to initiate tubular string buckling are calculated by substituting $\eta_{0}=0$ into Eq. (103)-(106),

$$
\begin{aligned}
& \eta_{5,2 D, \text { max }}=2.6012 \\
& \eta_{5,3 D, \text { max }}=4.3758 \\
& \eta_{5, \text { con, max }}=6.1864 \\
& \eta_{5, \text { hel, max }}=9.2453
\end{aligned}
$$

The lower limits of critical WOBs for tubular string buckling are respectively obtained by substituting $\eta_{0}=-\infty$ into Eq. (103)-(106),

$$
\begin{aligned}
& \eta_{5,2 D, \text { min }}=1.8546 \\
& \eta_{5,3 D, \text { min }}=3.9494 \\
& \eta_{5, \text { con, min }}=5.2497 \\
& \eta_{5, \text { hel, min }}=8.3954
\end{aligned}
$$

From Eq. (107) and (108) we can see that the variation amplitude of critical WOB due to WOH is less than 1. 


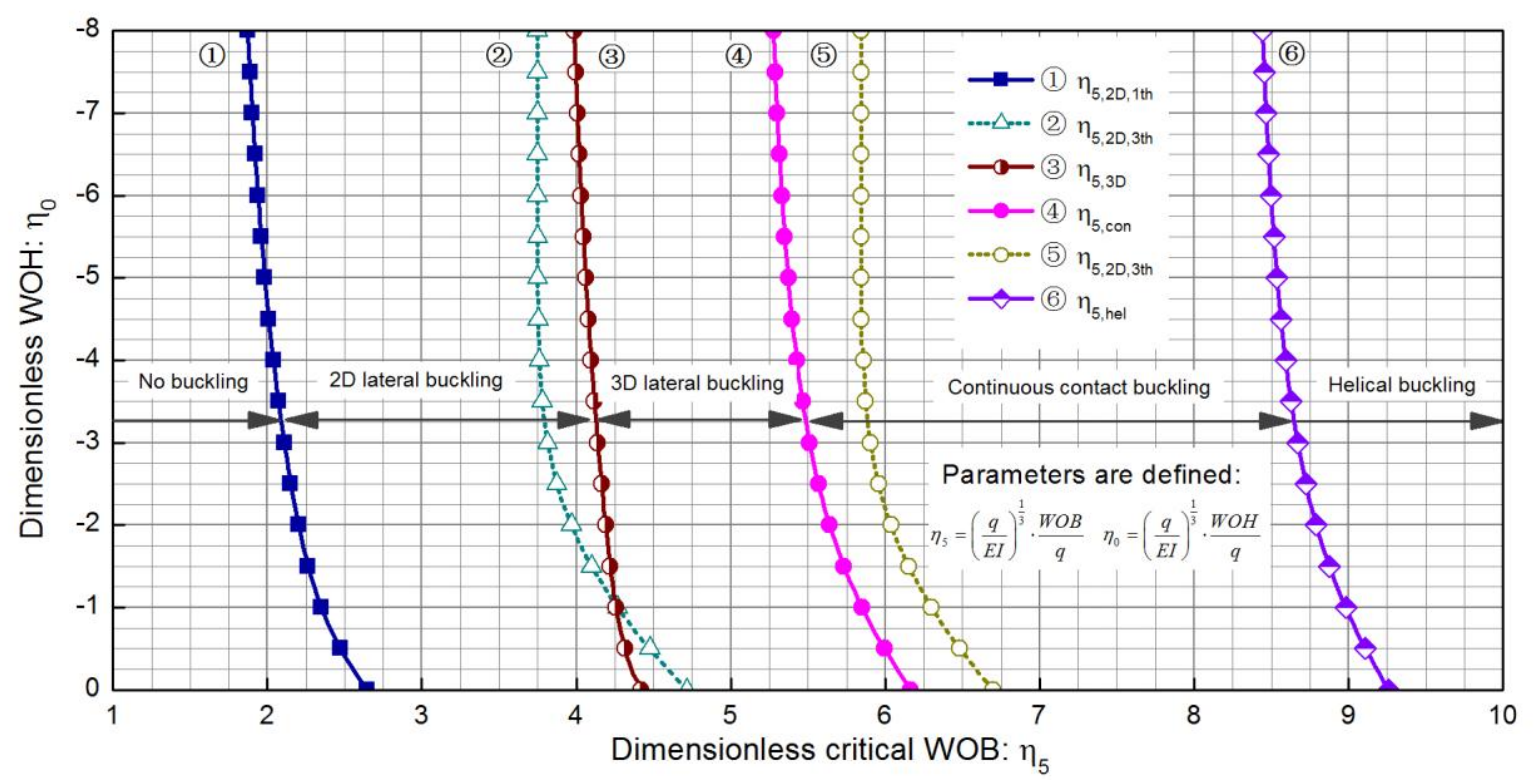

Fig. 9. Critical WOBs of different buckling modes under different WOHs.

The corresponding critical buckling loads in engineering unit are expressed by

$$
F_{\text {flag }}=\eta_{5, \text { flag }} \cdot\left(E I q^{2}\right)^{\frac{1}{3}}
$$

Table 4 shows the minimum critical buckling loads for several kinds of tubular strings. The critical helical bucking loads $F_{\text {hel }}$ for the coiled tubing (CT) are smaller than the threshold WOB of rock breaking. Therefore, it is inevitable that helical buckling occurs in vertical wellbore for coiled tubing drilling. The results show that drill pipes (DP) also cannot inhibit the occurrence of helical buckling. From Eq. (109) we can see that the critical buckling loads can be increased by increasing the outer diameter or the thickness of the tubular string. Thus, heavy weight drill pipe (HWDP) is more favorable for weakening the helical buckling. However, HWDP becomes incompetent under high value of WOB. To overcome this shortcoming, drilling collar (DC) is often adopted at the bottom of the entire tubular string. Although the helical buckling can be eliminated by using large-diameter and high-thickness tubular strings, it is usually difficult to avoid the lowest buckling mode-2D lateral buckling.

Table 4-Minimum critical buckling loads for several kinds of tubular strings

\begin{tabular}{ccccccccc}
\hline Type & $\begin{array}{c}\text { OD } \\
(\mathrm{mm})\end{array}$ & $\begin{array}{c}\mathrm{ID} \\
(\mathrm{mm})\end{array}$ & $\begin{array}{c}\mathrm{q} \\
(\mathrm{N} / \mathrm{m})\end{array}$ & $\begin{array}{c}\mathrm{EI} \\
\left(\mathrm{N} \times \mathrm{m}^{2}\right)\end{array}$ & $\begin{array}{c}\mathrm{F}_{2 \mathrm{D}} \\
(\mathrm{kN})\end{array}$ & $\begin{array}{c}\mathrm{F}_{3 \mathrm{D}} \\
(\mathrm{kN})\end{array}$ & $\begin{array}{c}\mathrm{F}_{\text {con }} \\
(\mathrm{kN})\end{array}$ & $\begin{array}{c}\mathrm{F}_{\text {hel }} \\
(\mathrm{kN})\end{array}$ \\
\hline CT & 50.8 & 44.45 & 36 & $2.827 \mathrm{E}+04$ & 0.62 & 1.32 & 1.75 & 2.80 \\
CT & 73 & 64.1 & 73 & $1.181 \mathrm{E}+05$ & 1.59 & 3.39 & 4.51 & 7.21 \\
DP & 73 & 54.6 & 141 & $2.002 \mathrm{E}+05$ & 2.94 & 6.26 & 8.32 & 13.30 \\
DP & 127 & 108.6 & 260 & $1.242 \mathrm{E}+06$ & 8.13 & 17.30 & 23.00 & 36.78 \\
HWD & 127 & 76.2 & 620 & $2.323 \mathrm{E}+06$ & 17.85 & 38.02 & 50.54 & 80.82 \\
DC & 127 & 57.2 & 772 & $2.559 \mathrm{E}+06$ & 21.35 & 45.46 & 60.42 & 96.63 \\
DC & 177.8 & 57.2 & 1701 & $1.014 \mathrm{E}+07$ & 57.22 & 121.84 & 161.96 & 259.01 \\
\hline
\end{tabular}

\section{(2) Contact states}

At the initial no buckling state, the tubular string remains in vertical line and does not touch the wellbore. If the 
axial force reaches the critical 2D lateral buckling, the tubular string deviates from the vertical line configuration and touches the wellbore at one single point. Fig. 10 shows the contact state between the tubular string and wellbore under the critical 2D lateral buckling condition, in which the dimensionless vertical distance is defined with the ground hook as the origin:

$$
\zeta=\frac{\eta-\eta_{0}}{\eta_{5}-\eta_{0}}
$$

From Eq. (110) we know $\zeta_{0}=0$ corresponds to the ground hook while $\zeta_{5}=1$ the drill bit.

Unlike the weightless tubular string, the tubular weight leads to the asymmetrical deflection curve of the tubular string. The contact point $\left(\zeta_{3}\right)$ between the tubular string and wellbore is on the lower half rather than the middle of the entire tubular string. The contact point divides the total tubular string into top suspended section and bottom suspended section. With the increase of WOH $\left(\eta_{0}\right)$, the percentage of the bottom suspended section increases and tends to 0.5. The fitting formula of the vertical depth of the contact point is expressed by

$$
\eta_{3,2 D}=-0.0586+1.4374 e^{0.3331 \eta_{0}}
$$

For the critical 3D lateral buckling, the contact point is lower than the critical 2D lateral buckling case. The fitting formula of the contact point depth is expressed by,

$$
\eta_{3,3 D}=2.5045+0.5065 e^{0.1453 \eta_{0}}
$$

If the hook load increases to be 0 , the percentage of the bottom suspended section to the entire tubular string is about 0.5 .

For the critical continuous contact buckling in Fig. 12, the entire tubular string is divided into four parts: the top, second, third and bottom suspended sections. The three contact points among the four suspended sections are calculated with Eq. (99), (100) and (101) while letting $\eta_{3}=\eta_{2}$. The lengths of the top and second suspended sections are usually larger than that of the third and bottoms sections due to the effect of tubular gravity. To be specific, the top suspended section is longer than the top suspended section when $\mathrm{WOH}$ is relatively large, for example $\eta_{0}>-4$. However, when WOH becomes smaller, the top suspended section is shorter than the top suspended section. The third suspended section is always longer than the bottom suspended section. Meanwhile, with the decrease of $\mathrm{WOH}\left(\eta_{0}\right)$, the three contact points moves downward to the well bottom.

For the critical helical buckling in Fig. 13, the entire tubular string is divided into five parts: the top, second, third and bottom suspended sections and the middle continuous contact section. The four contact points among the five sections are calculated with Eq. (99), (100), (101) and $\frac{\sqrt{2}}{3} \eta_{3}^{\frac{3}{2}}-\frac{\sqrt{2}}{3} \eta_{2}^{\frac{3}{2}}=2 \pi$. Similar to the critical continuous contact case, the four contact points move downward to the well bottom with the decrease of $\mathrm{WOH}\left(\eta_{0}\right)$. Note that the length of the continuous contact section is about twice of that of the third and bottom suspended sections. If the WOH is increased to be 0 , the continuous contact section occupies $40 \%$ of the total tubular string. 


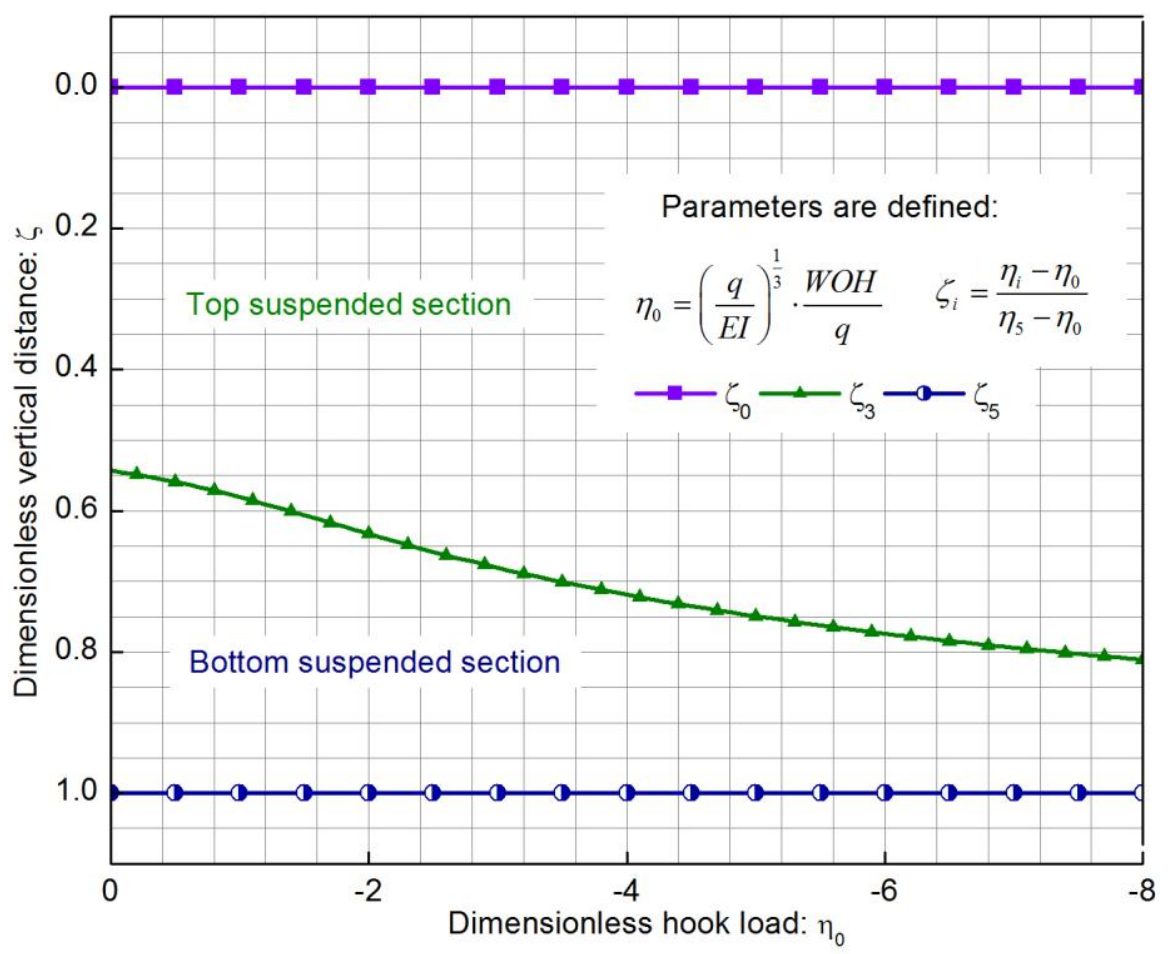

Fig. 10. Contact state of critical 2D lateral buckling under different WOHs

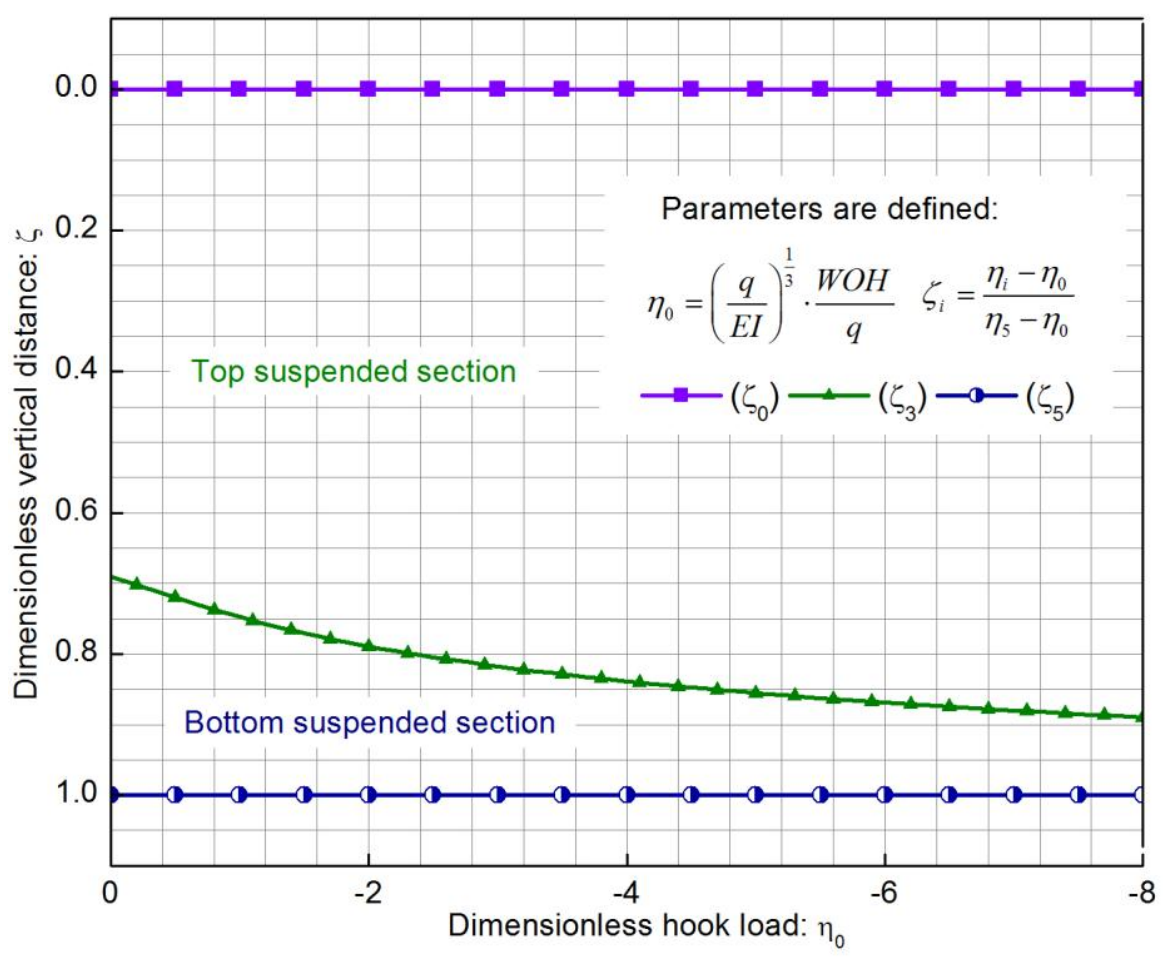

Fig. 11. Contact state of critical 3D lateral buckling under different WOHs 


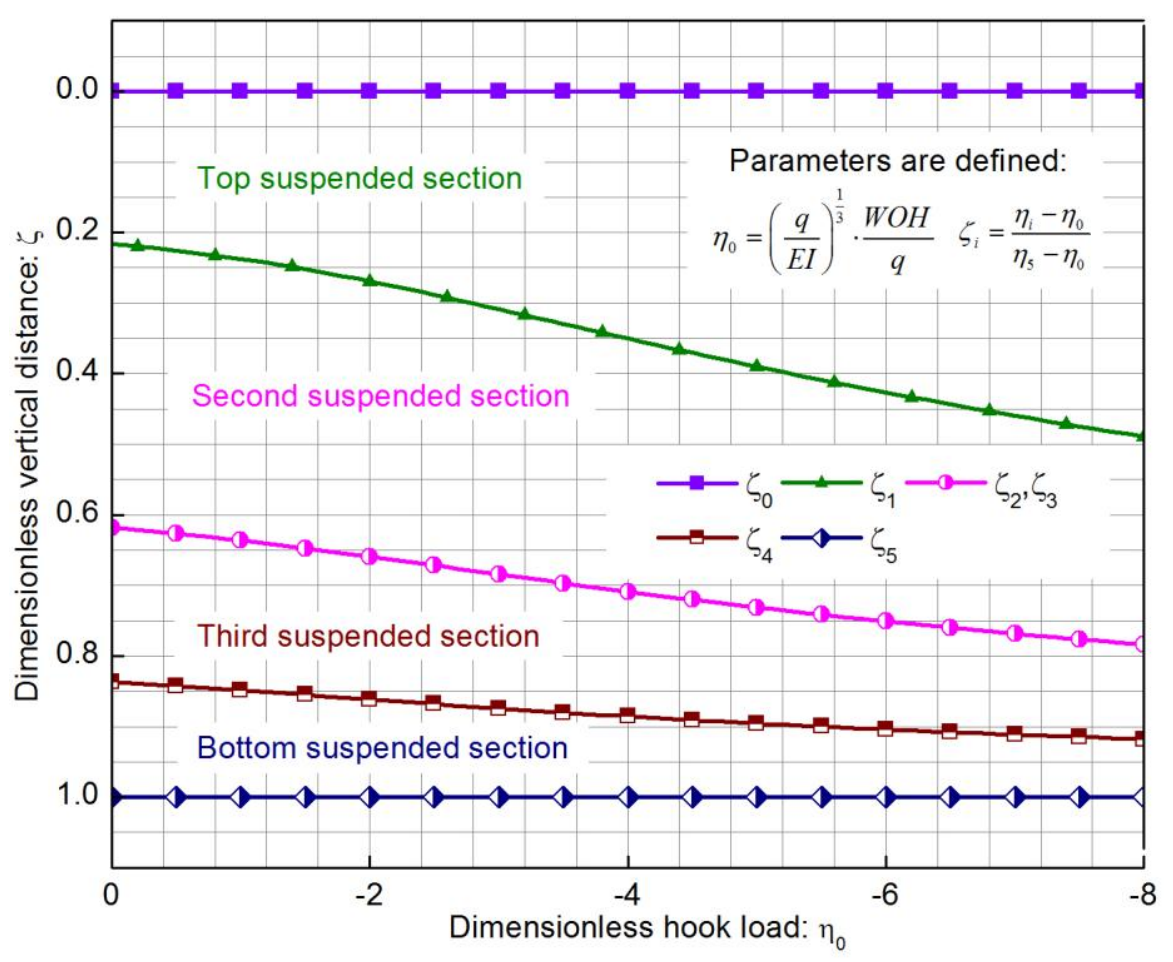

Fig. 12. Contact state of critical continuous contact buckling under different WOHs

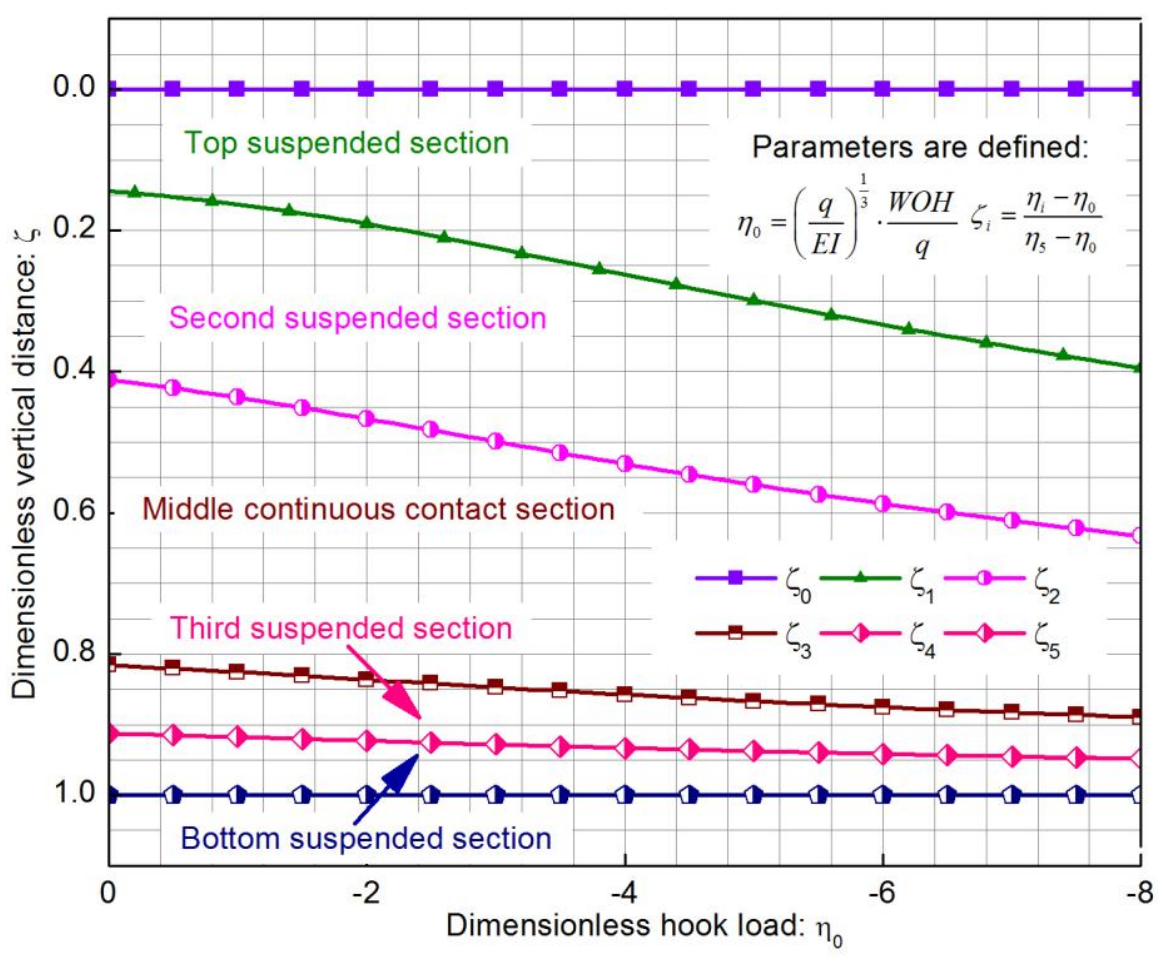

Fig. 13. Contact state of critical helical buckling under different WOHs

In the following analysis, the transition process of contact state with the increase of axial force is studied. Fig. 14 and Fig. 15 respectively show the contact states under different WOBs when WOH satisfying $\eta_{0}=-6$ and $\eta_{0}=0$. If WOB is 
lower than the critical 2D lateral buckling load (A), the tubular string does not touch the wellbore. At the 2D lateral buckling stage, and the tubular string is divided into two suspended sections and the contact point moves toward the well bottom with the increase of WOB. After WOB exceeds the critical 3D lateral buckling load (B), two another contact points are generated and deviates from the middle contact point with the increase of WOB. Thus, the total tubular string is divided into four suspended sections at this buckling stage. When WOB further achieves the critical continuous contact buckling load (C), the middle point starts to break into two contact points, between which a continuous contact section occurs. The continuous contact section grows longer but the other suspended sections become shorter with the increase of WOB at this buckling stage. When the continuous contact section is equal to a pitch of helix, the critical helical buckling load is obtained.

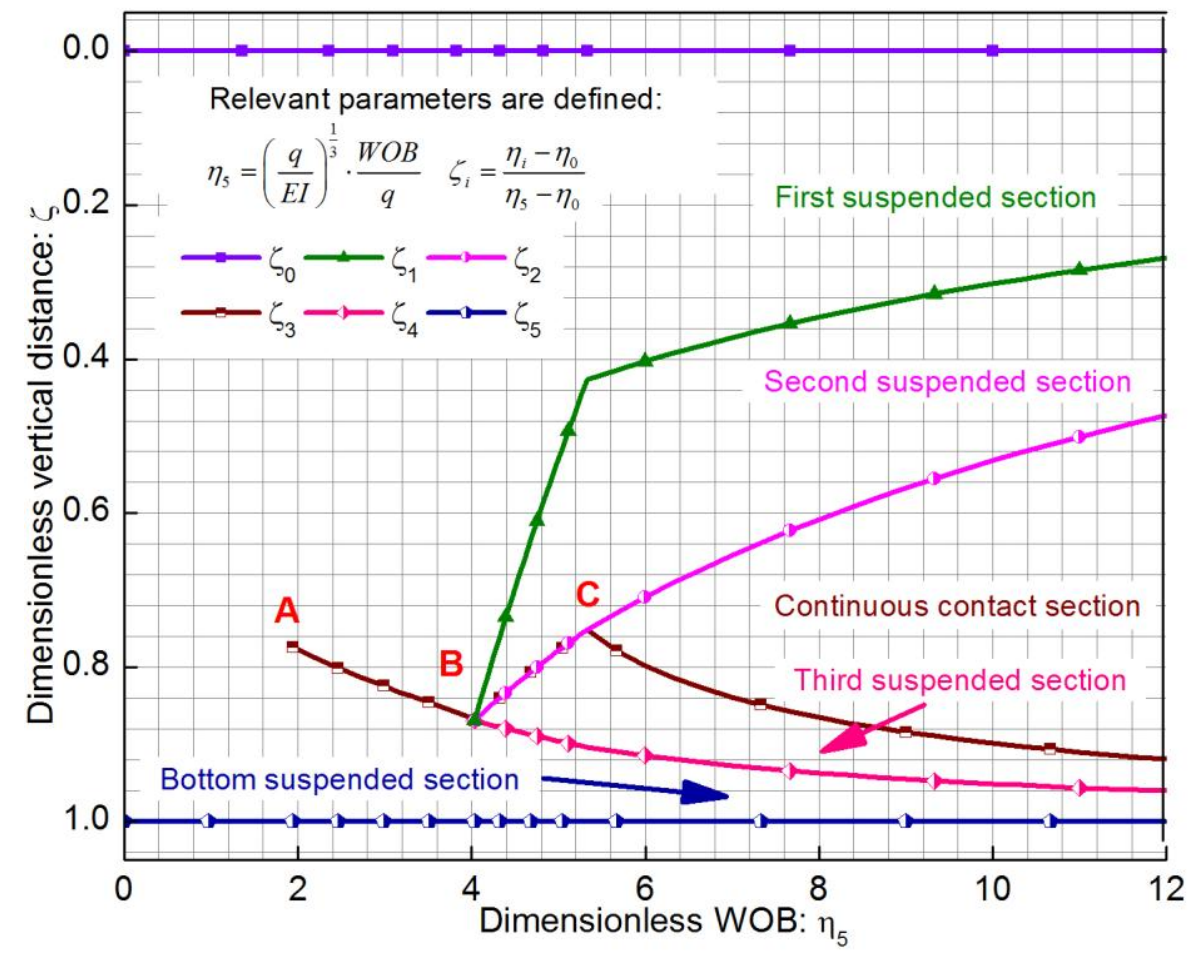

Fig. 14. Contact states under different WOBs when WOH satisfying $\eta_{0}=-6$. 


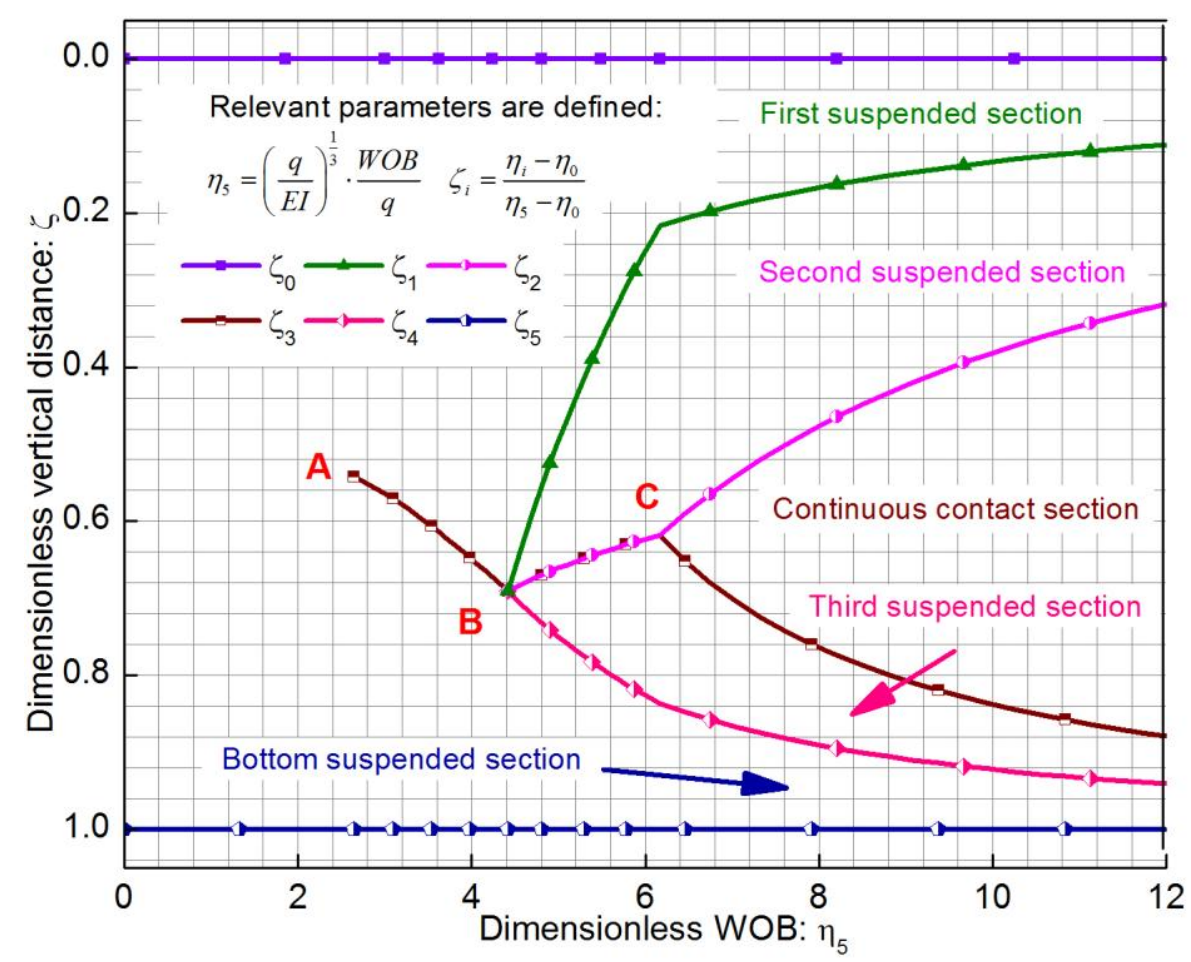

Fig. 15. Contact states under different WOBs when WOH satisfying $\eta_{0}=0$.

\section{(3) Deflection curves}

Fig. 16 and Fig. 17 show the deflection curves of the entire tubular string under different critical buckling conditions, where the dimensionless radial displacement and angular displacement are defined by:

$$
\hat{r}=\frac{\sqrt{\mu^{2}+\lambda^{2}}}{\delta_{c}} \quad \theta=\arctan \frac{\lambda}{\mu}
$$

At the critical 2D lateral buckling condition, the tubular string buckles into a 2D curve and touches the wellbore at one single point. At the 2D lateral buckling stage, the contact point moves downward with the increase of axial force. When the WOB achieves the critical 3D lateral buckling load, the 2D configuration becomes unstable and the tubular string starts to buckle in the three-dimensional space. Note that the tubular string buckles from the second-order 2D lateral mode to 3D lateral mode under the case of $\eta_{0}=-6$, while the tubular string buckling goes directly from first-order 2D lateral mode to 3D lateral mode under the case of $\eta_{0}=0$. When WOB exceeds the critical continuous contact buckling load, the tubular string touches the wellbore at three single points. The angular displacement change of the entire tubular string under this critical condition is about $2 \pi$. When WOB reaches the critical helical buckling load, a pitch of helix is in continuous contact with the wellbore. Therefore, the angular displacement change of the total tubular string under this critical condition is about $4 \pi$.

By comparing the results in Fig. 16 and Fig. 17 we can see that the radial and angular displacements are closely related to $\mathrm{WOH}\left(\eta_{0}\right)$. The decrease of $\mathrm{WOH}$ weakens the deflection of the top section of the tubular string and makes the contact points between the tubular string and wellbore move downward. 

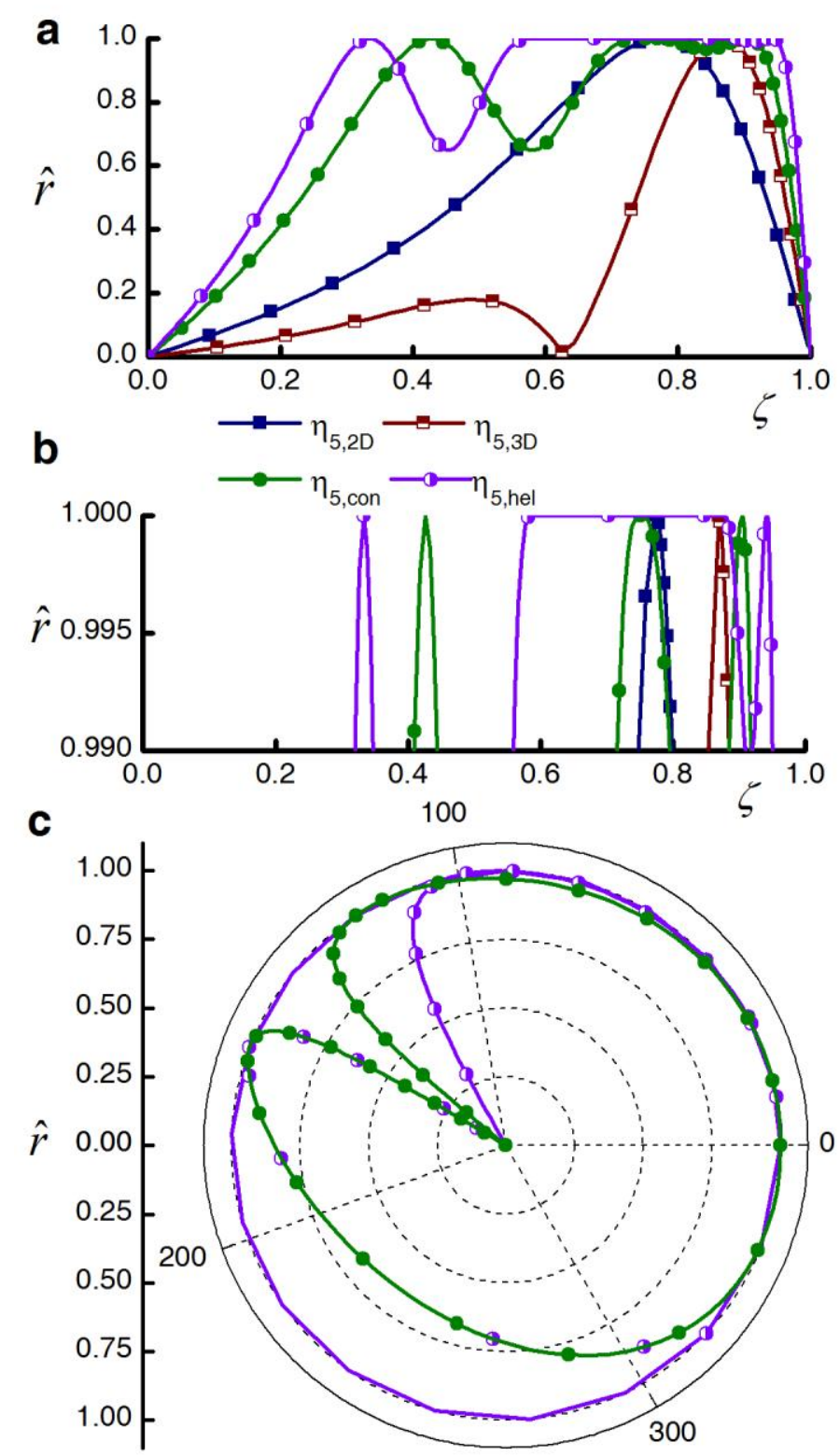

Fig. 16. Deflection curves under four critical conditions for $\eta_{0}=-6$. 


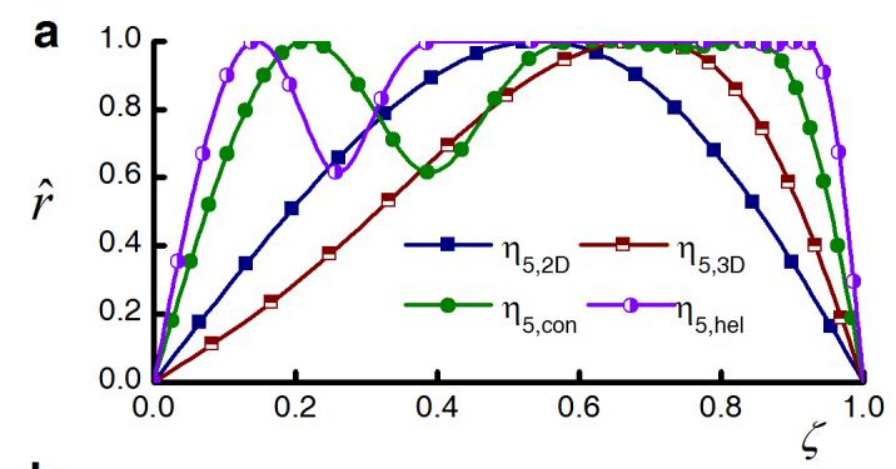

b
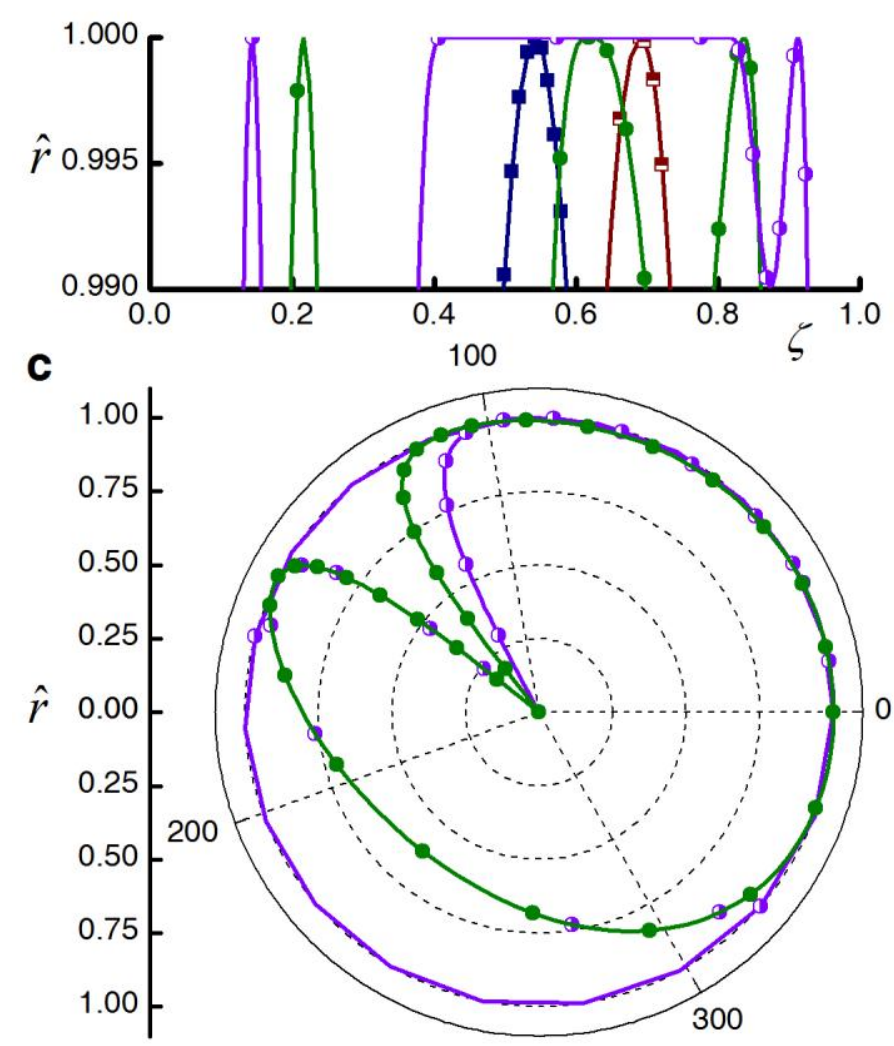

Fig. 17. Deflection curves under four critical conditions for $\eta_{0}=0$.

\section{(4) Well depth}

From the above analyses we know the tubular string buckling configuration can be determined by two terms: WOH $\left(\eta_{0}\right)$ and WOB $\left(\eta_{5}\right)$. These two terms are not independent to each other if the well depth is given in advance. Actually, it is more convenient for application with the combination of WOH \& well depth or WOB \& well depth to depict the tubular string buckling problem. When the friction force is neglected, the well depth can be determined by the difference between WOB and WOH:

$$
\eta_{L}=\eta_{5}-\eta_{0}
$$

Where $\eta_{L}=\left(\frac{q}{E I}\right)^{\frac{1}{3}} \cdot L$, in which $L$ is the well depth or the length of entire tubular string.

In the above analyses, the $\mathrm{WOH}\left(\eta_{0}\right)$ is taken as the key parameter affecting the critical buckling loads. Here, the 
critical loads $\left(\eta_{5, \text { flag }}\right)$ are expressed as the functions of $\eta_{L}$ by substituting Eq. (114) into Eq. (103)-(106), namely

$$
\begin{aligned}
& \eta_{5,2 D}=1.8546+0.7466 e^{0.3689\left(\eta_{5,2 D}-\eta_{L}\right)} \\
& \eta_{5,3 D}=3.9494+0.4264 e^{0.2783\left(\eta_{5,3 D}-\eta_{L}\right)} \\
& \eta_{5, \text { con }}=2.3451+1.4669 e^{0.3707\left(\eta_{5, \text { con }}-\eta_{L}\right)}+1.3089+3.0432 e^{-0.2753\left(2.3451+1.4669 e^{0.370\left(\eta_{5, c o n}-\eta_{L}\right)}\right)} \\
& \eta_{5, \text { hel }}=\left[3 \sqrt{2} \pi+\left(2.3451+1.4669 e^{0.3707\left(\eta_{s, h e l}-\eta_{L}\right)}\right)^{\frac{3}{2}}\right]^{\frac{2}{3}}+1.3089+3.0432 e^{-0.2753\left[3 \sqrt{2} \pi+\left(2.3451+1.46699 e^{0.370\left(\eta_{5, h e l}-\eta_{L} L\right.}\right)^{\frac{3}{2}}\right]^{\frac{2}{3}}}
\end{aligned}
$$

Fig. 18 shows the relationship between critical WOB and well depth for different buckling modes in Eq. (115)-(118), and the buckling modes can be determined when the well depth and WOB are given. The results indicate that the four critical WOBs decrease and tend to stable values with the increase of well depth. The left ends of the curves in Fig. 18 correspond to the maximum critical WOBs given in Eq. (107) to initiate 2D lateral, 3D lateral, continuous contact and helical buckling (points A, B, C and D in Fig. 18). Meanwhile, these four critical points also represent the minimum well depths to initiate these four kinds of tubular string buckling. When the well depth is rather large, for example $\eta_{L} \geq 14$, the minimum critical buckling loads are achieved.

In the above analyses, the WOB is the key factor to depict tubular string buckling problem. In fact, WOH is a more reasonable parameter than WOB for determining the buckling mode from the view of drilling control, for WOH can be controlled directly on the ground. At the initial state, the entire tubular string is suspended on the hook and the drill bit loses contact with the well bottom. Then, the $\mathrm{WOH}$ is equal to the negative value of tubular string weight, namely $\eta_{0}=-\eta_{L}$. If the tubular string is slacked off with the ground hook, then a part of the tubular string weight is supported by the wellbore bottom. In this process, WOH and WOB are both increasing and satisfy the expression $\eta_{0}=\eta_{5}-\eta_{L}$. The increase of WOH due to slacking off is defined as slack off weight (WSL), which is just identical with WOB $\left(\eta_{5}\right)$. The maximum WSL is achieved when the WOH is 0 , then WSL is equal to the weight of the entire tubular string $\left(\eta_{L}\right)$.

The buckling transition in Fig. 18 can be depicted from the view of WSL. With the increase of WSL, the tubular string goes through 2D lateral, 3D lateral, continuous contact buckling and helical buckling configurations. With the increase of well depth, the critical WSLs to initiate tubular string buckling decreases and tends to stable values.

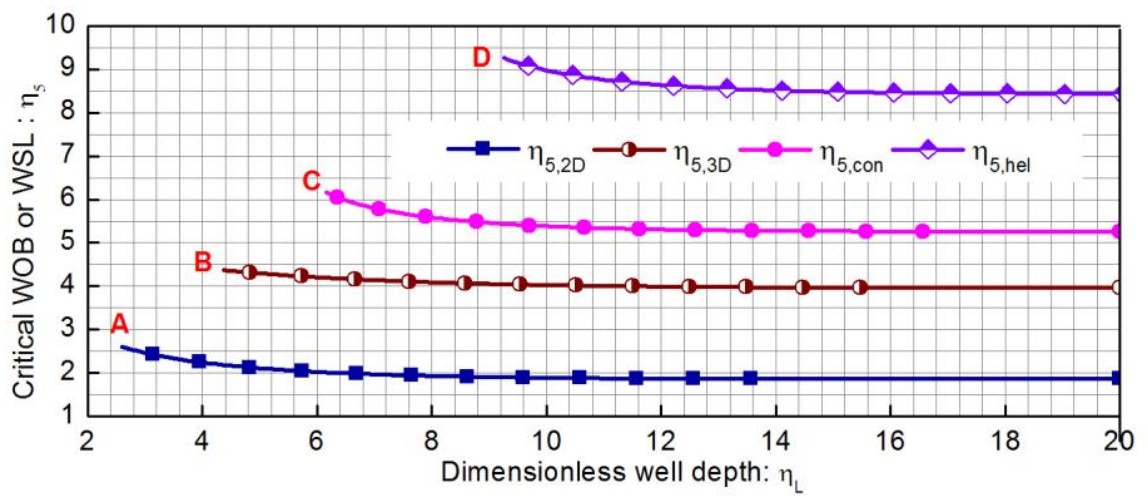

Fig. 18. Critical WOBs of different buckling modes under different well depths.

Taking the derivative of $\eta_{5, f l a g}$ with respect to $\eta_{L}$ in Eq. (115)-(118), we obtain the results shown in Fig. 19. Fig. 
19 also shows that $\frac{d}{d \eta_{L}} \eta_{5, f l a g}$ exponentially decreases and tends to 0 with the increase of $\eta_{L}$. Therefore, well depth has less and less effect on tubular string buckling with the increase of well depth and has no effect at last.

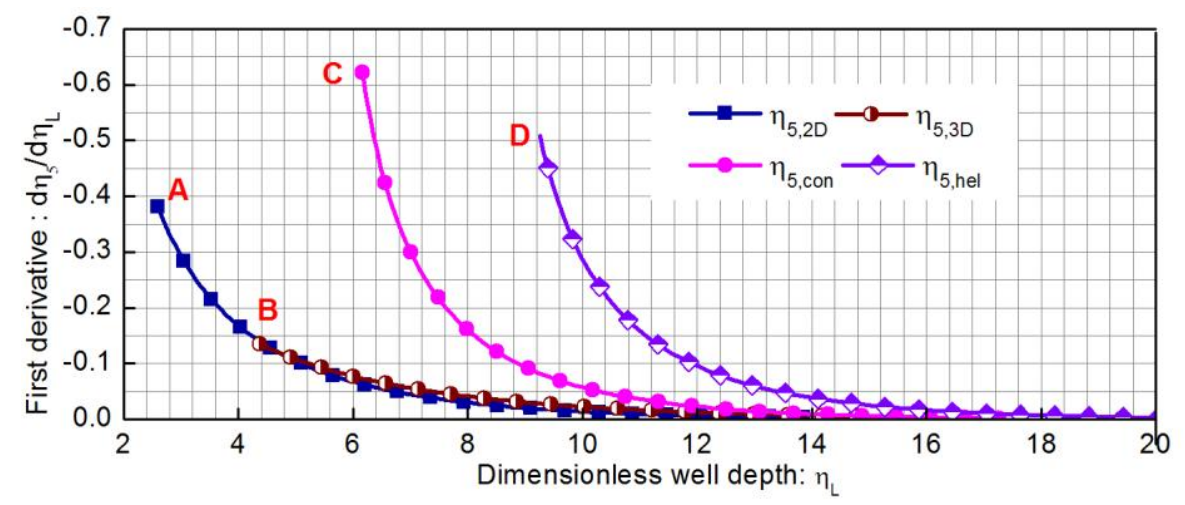

Fig. 19. First derivative of critical WOB with respect to well depth

\section{Axial force and torque transfer}

\subsection{Model description}

In the above analyses, four kinds of frictionless tubular string buckling are fully discussed. However, the friction force between the tubular string and wellbore cannot be neglected, especially when the tubular string is in continuous contact with the wellbore. Friction force can reduce the axial force and torque transfer along the tubular string, which may further impose constriction on drilling limit. In this section, we introduce the effect of friction force into the above tubular string buckling model in section 3. The axial force transfer in the sliding mode and the torque transfer in the rotating mode are respectively studied. To simplify the analysis, the following assumptions are adopted here:

(1) Friction forces on discretely contact points between the tubular string and wellbore are neglected.

(2) Friction force on the continuous contact section is included.

(3) In the sliding mode, the direction of friction force is along the wellbore axis; in the rotating mode, the friction force is in the circumferential direction.

According to assumption (1), the friction forces at the 2D and 3D lateral buckling stages are neglected. The friction forces on the suspended sections at the continuous contact buckling and helical buckling stages are also neglected. According to assumption (2), the effect of friction force on axial force and torque transfer on the continuous contact section should be included. In a word, the suspended sections are considered to be frictionless and friction force only affects the continuous contact section. According to assumption (3), only the axial force transfer problem is studied in sliding mode. For the rotating mode, the axial friction force is neglected, so the axial force transfer is identical with the frictionless case. Thus, only the torque transfer problem is studied in the rotating mode.

\subsection{Axial force transfer}

As is shown in Eq. (68), the dimensionless axial force $\xi$ is identical with the dimensionless vertical distance $\eta$ in the frictionless case. However, the term $\xi$ may not be equal to $\eta$ due to the effect of friction force in continuous contact buckling and helical buckling stages. In the following analyses, $\eta$ denotes the dimensionless vertical distance and $\xi$ the dimensionless axial force. To be specific, the terms $\eta_{0}-\eta_{1}, \eta_{1}-\eta_{2}, \eta_{3}-\eta_{4}$ and $\eta_{4}-\eta_{5}$ respectively denote the top, second third and bottom suspended sections and $\eta_{2}-\eta_{3}$ denotes the continuous contact section. The 
terms $\xi_{0}, \xi_{1}, \xi_{2}, \xi_{3}, \xi_{4}$ and $\xi_{5}$ respective represent the dimensionless axial forces on the points $\eta_{0}, \eta_{1}, \eta_{2}, \eta_{3}$, $\eta_{4}$ and $\eta_{5}$, where the following expressions are satisfied:

$$
\xi_{0}=\eta_{0} \quad \xi_{1}=\eta_{1} \quad \xi_{2}=\eta_{2} \quad \xi_{4}-\xi_{3}=\eta_{4}-\eta_{3} \quad \xi_{5}-\xi_{3}=\eta_{5}-\eta_{3}
$$

From Eq. (119) we know that the axial force transfer will be completely determined if the relationship between $\xi_{3}$ and $\eta_{3}$ is further given.

In the continuous contact section, the contact force between the tubular string and wellbore is calculated by (Gao, 2006)

$$
N=\operatorname{EIr}_{c}\left[4 \frac{d \theta}{d z} \frac{d^{3} \theta}{d z^{3}}+3\left(\frac{d^{2} \theta}{d z^{2}}\right)^{2}-\left(\frac{d \theta}{d z}\right)^{4}\right]+M_{T}\left[\left(\frac{d \theta}{d z}\right)^{3}-\frac{d^{3} \theta}{d z^{3}}\right]+\operatorname{Fr}_{c}\left(\frac{d \theta}{d z}\right)^{2}
$$

The dimensionless form of Eq. (120) is expressed by

$$
n=\delta_{c}\left[4 \frac{d \theta}{d \eta} \frac{d^{3} \theta}{d \eta^{3}}+3\left(\frac{d^{2} \theta}{d \eta^{2}}\right)^{2}-\left(\frac{d \theta}{d \eta}\right)^{4}+m\left(\frac{d \theta}{d \eta}\right)^{3}-m \frac{d^{3} \theta}{d \eta^{3}}+\xi\left(\frac{d \theta}{d \eta}\right)^{2}\right]
$$

Where the dimensionless contact force is defined by $n=N / q$.

For the case of zero friction and torque, the contact force is obtained by substituting Eq. (68) and (69) into Eq. (121),

$$
n=\delta_{c}\left(-\frac{1}{8 \eta}+\frac{\eta^{2}}{4}\right)
$$

Considering the above expression is satisfied on the continuous contact section $\eta>\eta_{2}$ in which $\eta_{2} \geq 2.3451$ from Eq. (100), the first term on the right hand of Eq. (122) $\left(-\frac{\delta_{c}}{8 \eta}\right)$ can be neglected. The neglected term just corresponds to the high-order derivatives including $\frac{d^{3} \theta}{d \eta^{3}}, \frac{d^{2} \theta}{d \eta^{2}}$ in Eq. (121). Here, we extend this conclusion to the case with friction fore, and Eq. (121) is simplified into

$$
n=\delta_{c}\left[-\left(\frac{d \theta}{d \eta}\right)^{4}+\xi\left(\frac{d \theta}{d \eta}\right)^{2}\right]
$$

Substituting Eq. (67) into Eq. (123), we obtain

$$
n=\frac{\delta_{c} \xi^{2}}{4}
$$

The differential equation depicting the axial force transfer in vertical wellbores is

$$
\frac{d F}{d s}=q-\mu_{f} \cdot N
$$

Where $\mu_{f}$ is the friction factor between the tubular string and wellbore.

The dimensionless form of Eq. (125) is expressed by

$$
\frac{d \xi}{d \eta}=1-\frac{\mu_{f} \delta_{c}}{4} \cdot \xi^{2}
$$

The general solution of Eq. (126) is equal to

$$
\xi(\eta)=\frac{2}{\sqrt{\mu_{f} \delta c}} \tanh \left(\frac{1}{2} \eta \sqrt{\mu_{f} \delta_{c}}+\frac{1}{2} C \sqrt{\mu_{f} \delta_{c}}\right)
$$


Where $C$ is an integral constant. Considering that the expression $\xi_{2}=\eta_{2}$ is satisfied at the starting point of continuous contact section, Eq. (127) is further transformed into

$$
\xi(\eta)=\frac{2}{\sqrt{\mu_{f} \delta c}} \tanh \left(\frac{1}{2}\left(\eta-\eta_{2}\right) \sqrt{\mu_{f} \delta_{c}}+\operatorname{arctanh}\left(\frac{1}{2} \eta_{2} \sqrt{\mu_{f} \delta_{c}}\right)\right)
$$

With the above equation, the relationship between $\xi_{3}$ and $\eta_{3}$ is determined as follows:

$$
\xi_{3}=\frac{2}{\sqrt{\mu_{f} \delta c}} \tanh \left(\frac{1}{2}\left(\eta_{3}-\eta_{2}\right) \sqrt{\mu_{f} \delta_{c}}+\operatorname{arctanh}\left(\frac{1}{2} \eta_{2} \sqrt{\mu_{f} \delta_{c}}\right)\right)
$$

Combining Eq. (119) and (129), the axial force transfer along the tubular string can be obtained. The axial force transfer is calculated by the following steps:

(1) The terms including $\xi_{0-2}$ and $\eta_{1-2}$ are calculated with Eq. (99), (100) and Eq. (119) when $\eta_{0}$ is given in advance.

(2) The term $\xi_{3}$ is obtained with Eq. (129) when $\eta_{3}$ is given.

(3) The terms $\xi_{4-5}$ is obtained with the modified Eq. (101) and (102):

$$
\begin{aligned}
& \xi_{4}=\xi_{3}+0.7131+2.5651 e^{-0.3602 \xi_{3}} \\
& \xi_{5}=\xi_{3}+1.3089+3.0432 e^{-0.2753 \xi_{3}}
\end{aligned}
$$

(4) The terms $\eta_{4-5}$ are solved with Eq. (119).

In the section 3, the term $\eta_{5}$ is defined as the slack off weight (WSL). If the friction force is considered, the definition of $\eta_{5}$ is still satisfied. According to the above calculation procedures, the WSL is equal to

$$
\eta_{5}=2.3451+1.4669 e^{0.370 \eta_{0}}+\Delta \eta_{23}+1.3089+3.0432 \exp \left[-0.2753 \frac{2}{\sqrt{\mu_{f} \delta_{c}}} \tanh \left(\frac{1}{2} \Delta \eta_{23} \sqrt{\mu_{f} \delta_{c}}+\operatorname{arctanh}\left(\frac{1}{2}\left(2.3451+1.4669 e^{0.3707 \eta_{0}}\right) \sqrt{\mu_{f} \delta_{c}}\right)\right)\right]
$$

Where $\Delta \eta_{23}$ denotes the length of the continuous contact section. For the convenience of application, two terms including WSL $\left(\eta_{5}\right)$ and well depth $\left(\eta_{L}\right)$ are used to depict the axial force transfer problem. Combining Eq. (114) with Eq. (132), the two terms including $\Delta \eta_{23}$ and $\eta_{0}$ are calculated. Then, the dimensionless WOB is obtained by substituting $\Delta \eta_{23}$ and $\eta_{0}$ into Eq. (135),

$$
\begin{aligned}
\xi_{5}= & \frac{2}{\sqrt{\mu_{f} \delta_{c}}} \tanh \left(\frac{1}{2} \Delta \eta_{23} \sqrt{\mu \delta_{c}}+\operatorname{arctanh}\left(\frac{1}{2}\left(2.3451+1.4669 e^{0.3707 \eta_{0}}\right) \sqrt{\mu_{f} \delta_{c}}\right)\right)+1.3089 \\
& +3.0432 \exp \left[-0.2753 \frac{2}{\sqrt{\mu_{f} \delta_{c}}} \tanh \left(\frac{1}{2} \Delta \eta_{23} \sqrt{\mu_{f} \delta_{c}}+\operatorname{arctanh}\left(\frac{1}{2}\left(2.3451+1.4669 e^{0.3707 \eta_{0}}\right) \sqrt{\mu_{f} \delta_{c}}\right)\right)\right]
\end{aligned}
$$

Fig. 20 shows the axial force transfer on the tubular string in shallow wells when the tubular string is completely slacked off. If the friction force is neglected, namely $\mu \delta_{c}=0$, the dimensionless axial force is identical with the dimensionless vertical depth. If the friction force is not 0 , the axial force is lower than that under the frictionless case. With the increase of friction force $\mu$ or the radial clearance $\delta_{c}$, the axial force becomes smaller and the increase rate of axial force with respect to well depth decreases in the continuous contact section. Fig. 21 shows the axial force transfer in deep wells when the tubular string is completely slacked off. It is indicated that the friction forces reduce the increase rates of axial force and make axial forces tends to stable values with the vertical depth. According to Eq. (133) we obtain the maximum value of WOB transferred to the drill bit for a tubular string is completely slacked off in an infinite deep well, namely 


$$
\xi_{5}=\frac{2}{\sqrt{\mu_{f} \delta_{c}}}+1.3089+3.0432 \exp \left(-0.2753 \frac{2}{\sqrt{\mu_{f} \delta_{c}}}\right)
$$

Note that the maximum WOB decreases with the increase of the parameter $\mu \delta_{c}$.

Fig. 22 shows the change of WOB with the increase of WSL. The results show that the WSL cannot be sufficiently transferred to the drill bit due to the friction force between the tubular string and wellbore, especially for high friction factor and large radial clearance. The different between WSL and WOB, namely $\eta_{5}-\xi_{5}$ is just equal to the total friction force.

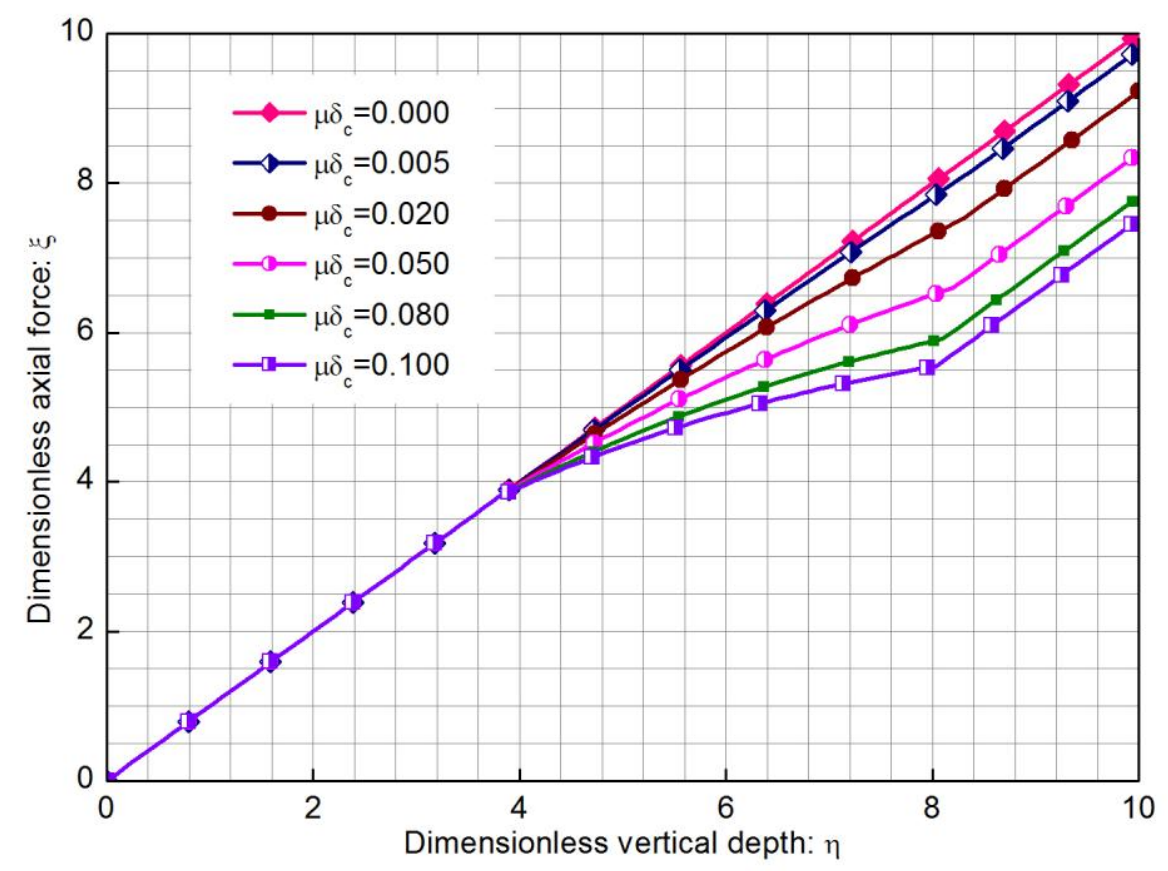

Fig. 20. Axial force vs. vertical distance under different values of $\mu \delta_{c}$ for shallow wells $\left(\eta_{5}=10\right.$ and $\left.\eta_{L}=10\right)$. 


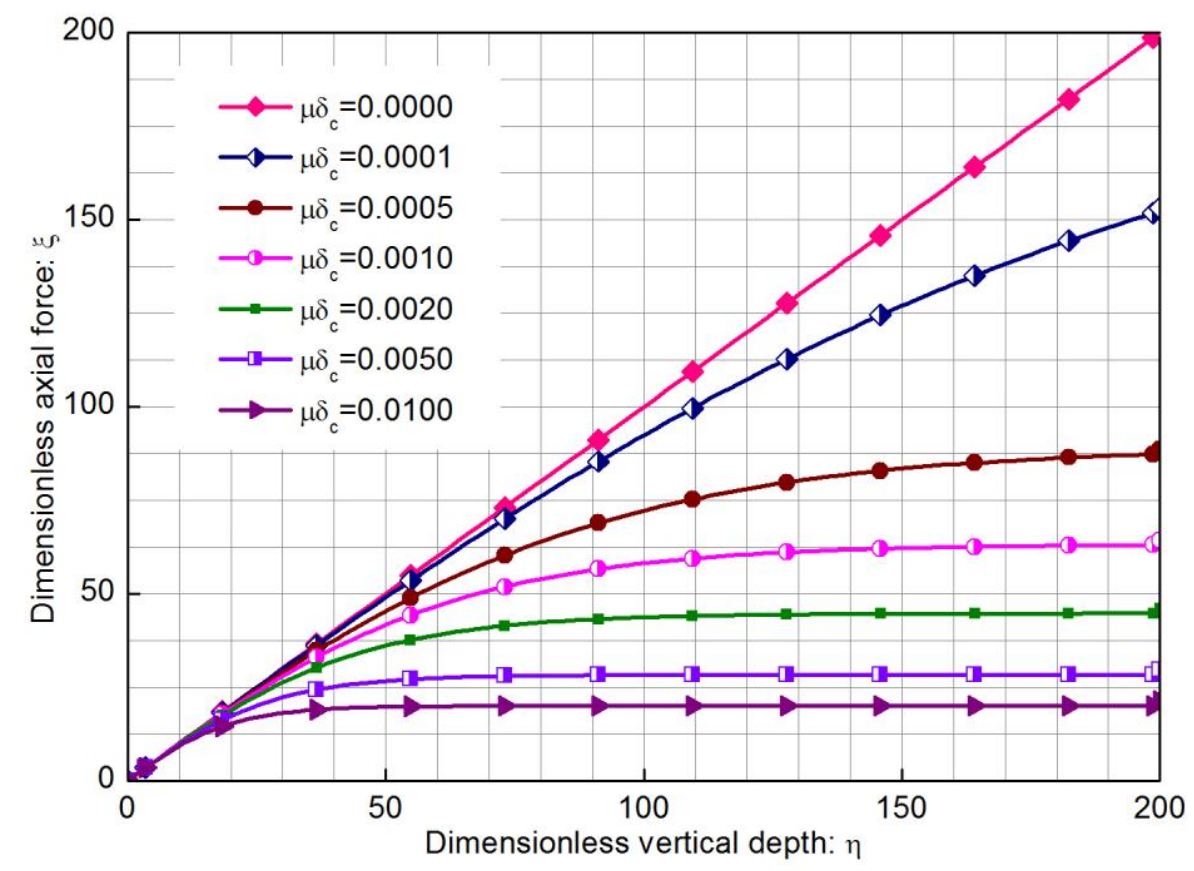

Fig. 21. Axial force vs. vertical distance under different values of $\mu \delta_{c}$ for deep wells $\left(\eta_{5}=200\right.$ and $\left.\eta_{L}=200\right)$.

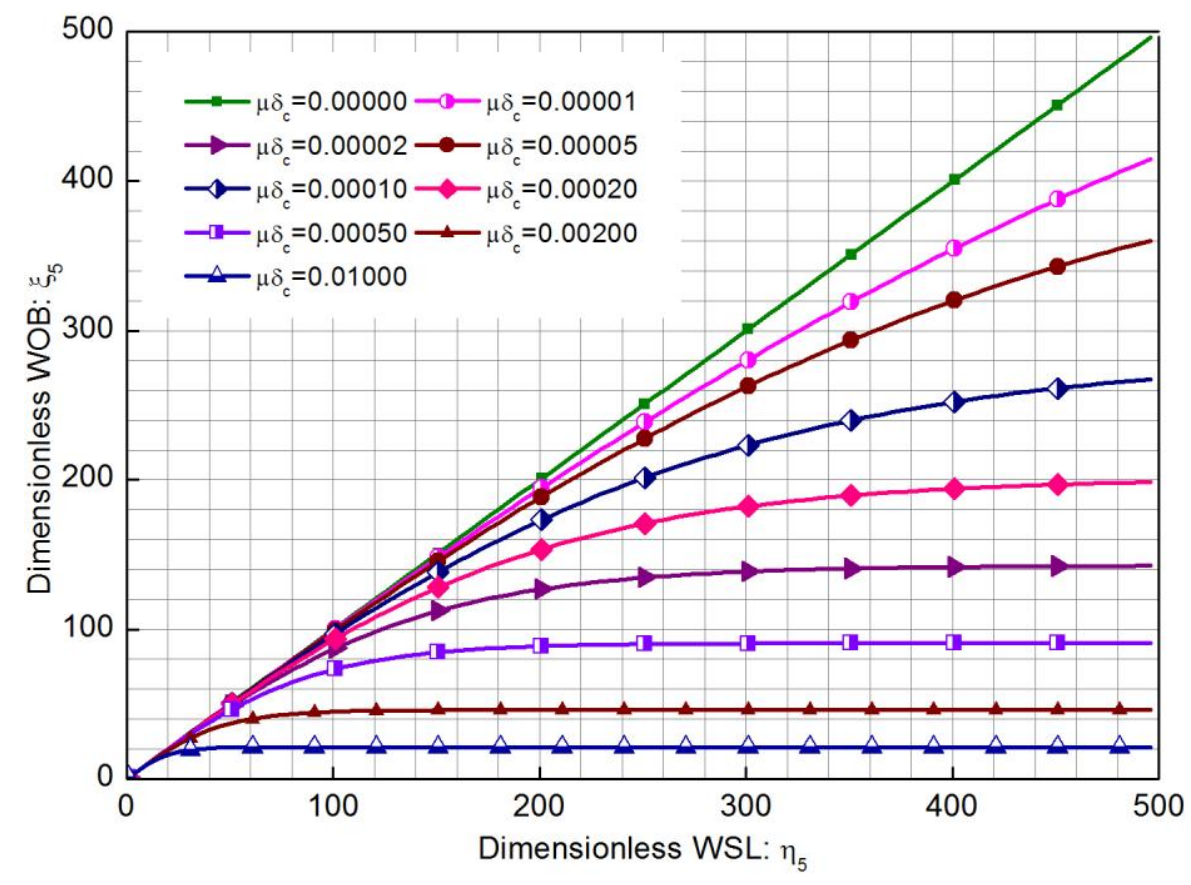

Fig. 22. WOB vs. WSL under different values of $\mu \delta_{c}\left(\eta_{L}=500\right)$.

\subsection{Torque transfer}

The terms $m_{0}, m_{1}, m_{2}, m_{3}, m_{4}$ and $m_{5}$ respective represent the dimensionless torques on the points $\eta_{0}, \eta_{1}$, $\eta_{2}, \eta_{3}, \eta_{4}$ and $\eta_{5}$. Because the friction forces on the suspended sections are neglected, the torque on key points satisfies:

$$
m_{0}=m_{2} \quad m_{1}=m_{2} \quad m_{4}=m_{3} \quad m_{5}=m_{3}
$$

From Eq. (135) we know that the torque transfer will be completely determined if the relationship between $m_{3}$ and 
$m_{2}$ is further given.

In the continuous contact section, the angular displacement under the action of torque is calculated from Eq. (66):

$$
\frac{d \theta}{d \eta}=\frac{3}{8} m \pm \frac{1}{8} \sqrt{9 m^{2}+32 \xi}
$$

Eq. (136) represents two helixes of different spiral directions. In the following analyses, only the component including "+" is considered. When the tubular string is rotated, the friction force is in the circumferential direction. Then, the axial force transfer is equal to the frictionless case, namely $\xi=\eta$. Thus, the angular displacement is written as

$$
\frac{d \theta}{d \eta}=\frac{3}{8} m+\frac{1}{8} \sqrt{9 m^{2}+32 \eta}
$$

Neglecting the higher derivatives of $\theta$ with respect to $\eta$, the contact force on the continuous contact section Eq. (121) is simplified into

$$
n=\delta_{c}\left[-\left(\frac{d \theta}{d \eta}\right)^{4}+m\left(\frac{d \theta}{d \eta}\right)^{3}+\eta\left(\frac{d \theta}{d \eta}\right)^{2}\right]
$$

Substituting Eq. (137) into Eq. (138) and retaining the liner term of m, we obtain the approximate dimensionless contact force:

$$
n=\delta_{c}\left(\frac{1}{4} \eta^{2}+\frac{\sqrt{2}}{4} \eta^{3 / 2} m\right)
$$

The torque transfer along the tubular string in vertical wellbores is calculated by

$$
\frac{d M_{T}}{d z}=-\mu_{f} \cdot N \cdot r_{d}
$$

Where $r_{d}$ the outer radius of the tubular string.

Introducing the dimensionless outer radius $\delta_{d}=\left(\frac{q}{E I}\right)^{\frac{1}{3}} \cdot r_{d}$ and substituting Eq. (139), Eq. (140) can be written into its dimensionless form:

$$
\frac{d m}{d \eta}=-\mu_{f} \delta_{d} \cdot n=-\mu_{f} \delta_{c} \delta_{d}\left(\frac{1}{4} \eta^{2}+\frac{\sqrt{2}}{4} \eta^{3 / 2} m\right)
$$

From Eq. (135) we know that the torque transfer will be determined if the relationship between $m_{3}$ and $m_{2}$ is further given. The solution of Eq. (141) under the condition of $m\left(\eta_{3}\right)=m_{3}$ is derived in Appendix B:

$$
m=\frac{\sqrt{2}}{2}\left(-\frac{\sqrt{2} \mu_{f} \delta_{c} \delta_{d}}{10}\right)^{-1 / 5} \mathrm{e}^{-\frac{\sqrt{2} \mu_{f} \delta_{c} \delta_{d}}{10} \eta^{5 / 2}}\left[\Gamma\left(\frac{6}{5},-\frac{\sqrt{2}}{10} \mu_{f} \delta_{c} \delta_{d} \eta_{3}^{5 / 2}\right)-\Gamma\left(\frac{6}{5},-\frac{\sqrt{2}}{10} \mu_{f} \delta_{c} \delta_{d} \eta^{5 / 2}\right)\right]+m_{3} \mathrm{e}^{\frac{\sqrt{2}}{10} \mu_{f} \delta_{c} \delta_{d}\left(\eta_{3}^{5 / 2}-\eta^{5 / 2}\right)}(142)
$$

Eq. (142) represents the torque transfer along the tubular string. The term $m_{2}$ can be written as the function of $m_{3}$ as follows:

$$
m_{2}=\frac{\sqrt{2}}{2}\left(-\frac{\sqrt{2} \mu_{f} \delta_{c} \delta_{d}}{10}\right)^{-1 / 5} \mathrm{e}^{-\frac{\sqrt{2} \mu_{j} \delta_{c_{d}}}{10} \eta_{2}^{5 / 2}}\left[\Gamma\left(\frac{6}{5},-\frac{\sqrt{2}}{10} \mu_{f} \delta_{c} \delta_{d} \eta_{3}^{5 / 2}\right)-\Gamma\left(\frac{6}{5},-\frac{\sqrt{2}}{10} \mu_{f} \delta_{c} \delta_{d} \eta_{2}^{5 / 2}\right)\right]+m_{3} \mathrm{e}^{\frac{\sqrt{2}}{10} \mu_{f} \delta_{c} \delta_{d}\left(\eta_{3}^{5 / 2}-\eta_{2}^{5 / 2}\right)}
$$

By combining Eq. (135) with (143) we can see that the torque transfer along the tubular string can be determined once the torque on bit (TOB, $m_{5}$ ) is given. In actual, TOB can be taken as the function of WOB and revolution per minute of the tubular string. Therefore, the torque transfer is coupled with the axial force transfer problem. 
Fig. 23 shows the torque distribution along the vertical distance for $\mathrm{m}_{5}=0.1$ under different values of $\mu \delta_{c} \delta_{\mathrm{d}}$. The value of torque decreases with the vertical depth due to the friction force between the tubular string and wellbore. The decrease rate of torque with respect to vertical depth increases due to the increase of contact force caused by the combination of axial force and torque. Overall, the torque along the entire tubular string increases with the increase of the parameter $\mu \delta_{\mathrm{c}} \delta_{\mathrm{d}}$. Therefore, larger value of $\mu \delta_{\mathrm{c}} \delta_{\mathrm{d}}$ corresponds to higher torque on rotary table (TOR) to rotate the down-hole tubular string. Fig. 24 shows the torque distribution along the tubular string for $\mu \delta_{c} \delta_{d}=0.0005$ under different values of $m_{5}$. The results show that the distributions of torque are shifted with the same amplitude of TOB $\left(\mathrm{m}_{5}\right)$.

Fig. 25 shows the relationship between TOR $\left(\mathrm{m}_{0}\right)$ and WSL $\left(\eta_{5}\right)$ under different values of $\mu \delta_{c} \delta_{\mathrm{d}}$. It is indicated that $\mathrm{m}_{0}$ increases exponentially with $\eta_{5}$. The combination of high WSL $\left(\eta_{5}\right)$ and large value of $\mu \delta_{\mathrm{c}} \delta_{\mathrm{d}}$ may make TOR $\left(\mathrm{m}_{0}\right)$ exceed the rated TOR of the drill rig or lead to tubular string failure, which is the limit of rotary drilling in vertical wells.

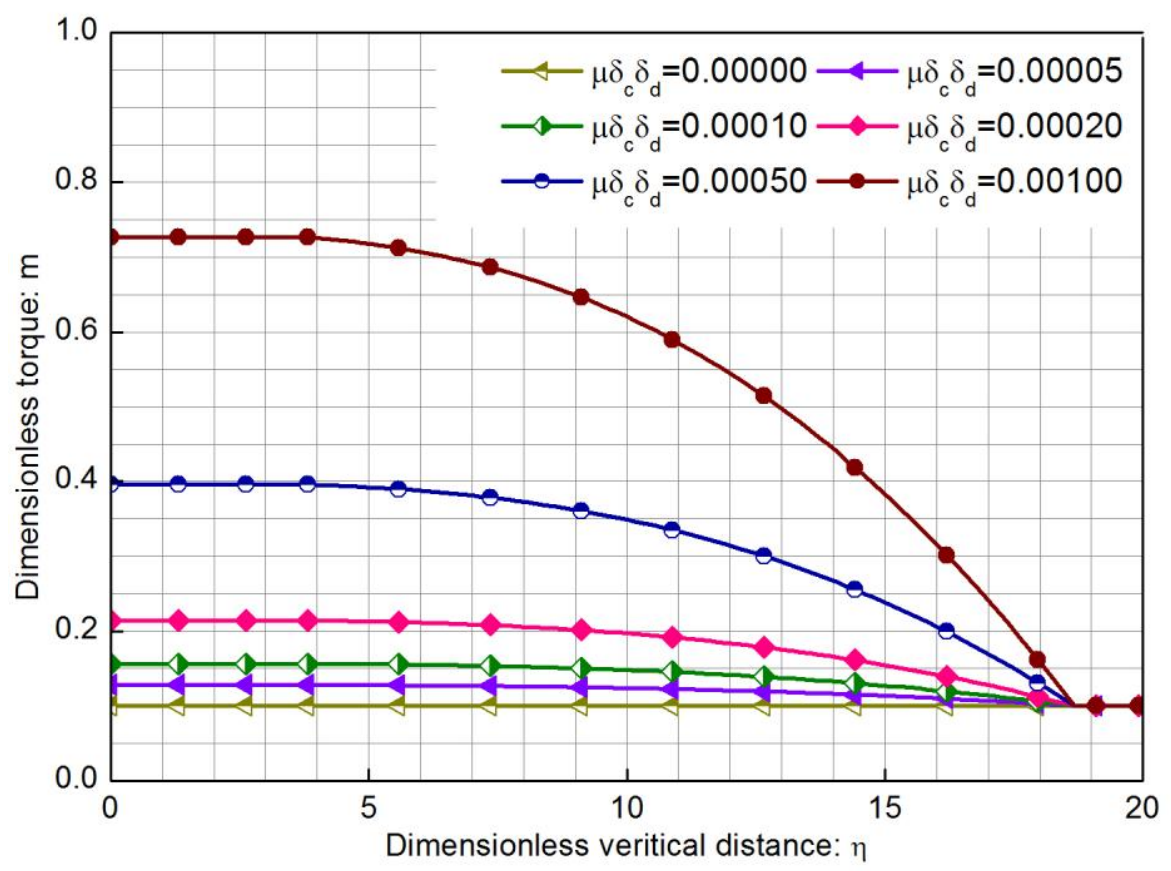

Fig. 23. Torque vs. vertical distance under different values of $\mu \delta_{c} \delta_{d}\left(\eta_{5}=20\right.$ and $\left.\eta_{L}=20\right)$. 


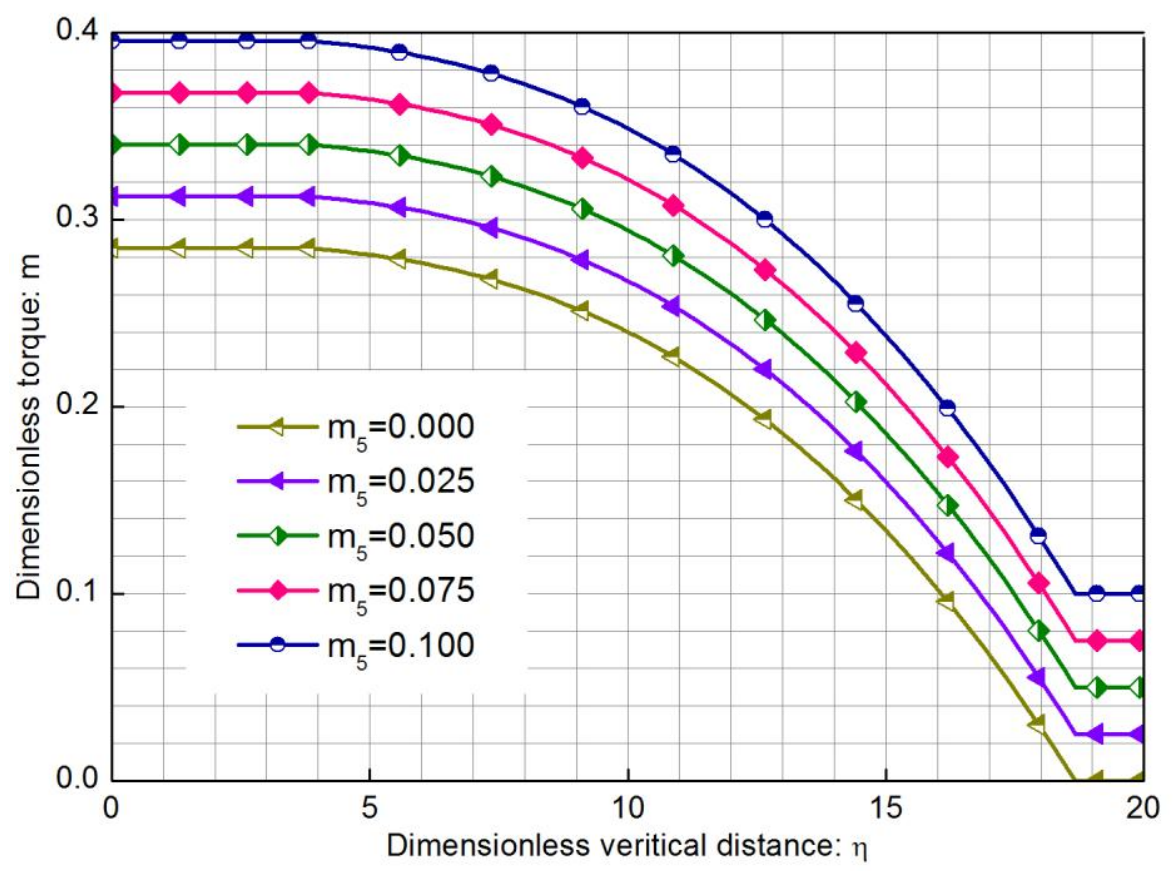

Fig. 24. Torque vs. vertical distance under different values of $m_{5}\left(\eta_{5}=10\right.$ and $\left.\eta_{L}=10\right)$.

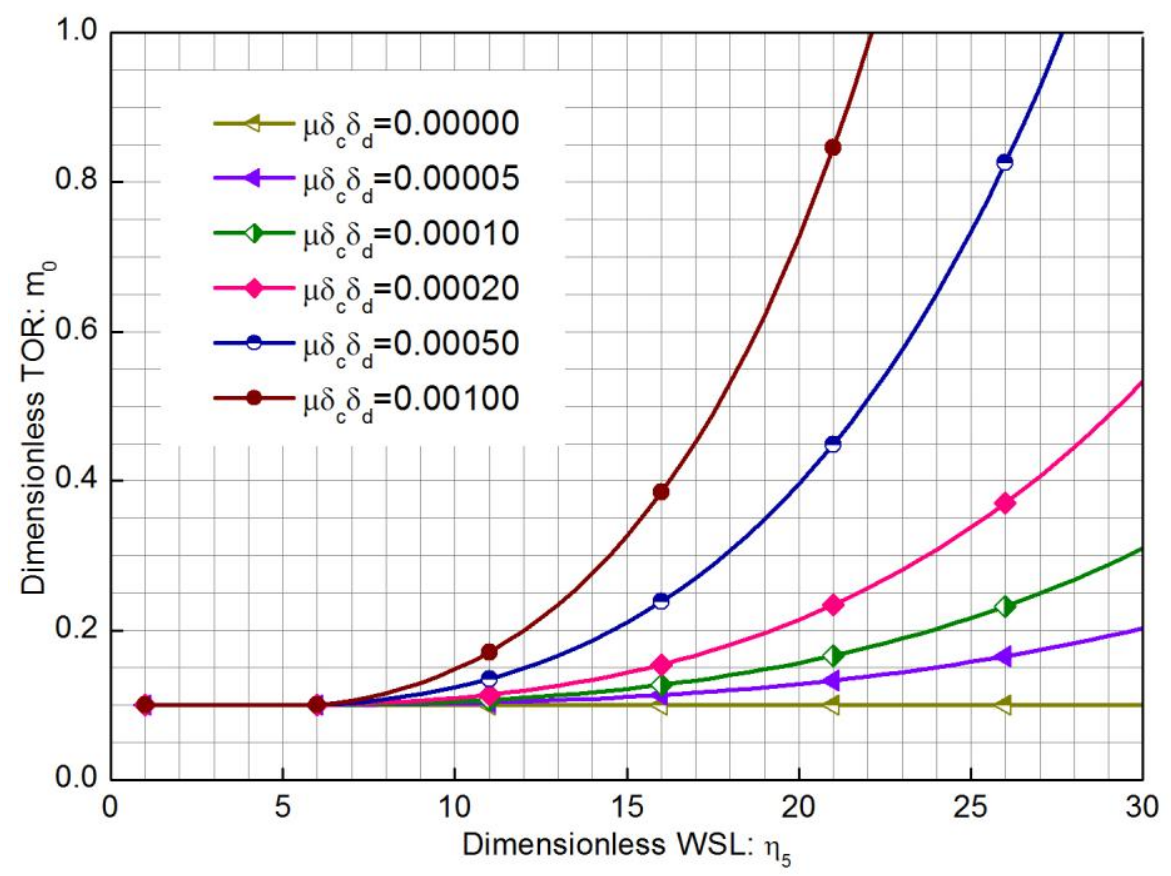

Fig. 25. Torque vs. WSL under different values of $\mu \delta_{c} \delta_{d}\left(\eta_{L}=30\right)$.

\section{Further discussions on tubular string buckling}

In this paper, quantitative models of the full buckling modes of tubular strings without and with weight in vertical wellbore are built. The tubular string goes through non-buckling, 2D lateral buckling, 3D lateral buckling, continuous contact buckling and helical bucking with the increase of axial force. Meanwhile, the contact status between the tubular string and wellbore is converted from no contact, point contact to continuous contact. For the weightless tubular string, 
the critical buckling loads are determined by the bending stiffness and the length of the tubular string. However, for the tubular string with weight, the critical buckling loads are also related to the tubular string weight per unit length. The symmetry of the buckling configuration is destroyed and the calculation complexity is greatly increased due to the effect of tubular string weight. In a word, the weight model can be seen as the generalized form of the weightless model.

The conventional BHA mechanical model is usually focused on the local portion of the tubular string below the tangency point. However, from the analysis in the weight model, the conventional definition of tangency point is not satisfied when continuous contact buckling or helical buckling occurs, and the deflection curve of the bottom suspended section is also affected by the above continuous contact section. Therefore, the existence of buckling is detrimental to the stability of the well tendency control. Meanwhile, the existence of buckling may greatly reduce the axial force and torque transfer along the tubular string. The coupling of buckling and friction force leads to the upper limit of WOB even if the WSL is increased to infinity. And, the value of TOR to rotate the tubular string under rather large WSL may exceed the maximum power of the drill rig or the torsional strength of the tubular string. To overcome these problems, some measures must be adopted to inhibit the buckling problem on the tubular string.

From the above buckling models, the critical buckling loads can be increased by adopting large-diameter and high-thickness tubular strings. However, the increase of the diameter and thickness increases the pick-up weight of the entire tubular string, which may require more powerful drilling rig in deep wells. The weightless model, which can also be taken as the buckling model of a local tubular string between two stabilizers, points out that the existence of stabilizers can greatly increase the critical buckling loads by comparing Table 2, Table 3 with Table 4. Therefore, installing stabilizers on the buckling-prone sections can not only increase the well tendency control, axial force and torque transfer, but also contribute to the reduction of the entire tubular string weight. In actual drilling process, tubular strings with several tapered portions and with discretely distributed connectors are two important measures to inhibit tubular string buckling. This paper only considers the buckling of a tubular string of the same size and with two adjacent connectors, so the next research should be extended to the tubular string with multiple tapered portions and connectors.

\section{Conclusions}

In this paper, the critical buckling loads and buckling configurations under different buckling modes for a tubular string in vertical wellbores are presented, and the axial force and torque transfer under the coupling effect of friction force and buckling is discussed. On the basis of the above research, the following achievements are obtained:

1. The tubular string in a vertical wellbore goes through non-buckling, 2D lateral buckling, 3D lateral buckling, continuous contact buckling and helical bucking with the increase of axial force. For the 2D lateral buckling, the entire tubular string is divided into two suspended sections by a single contact point. For the 3D lateral buckling, four suspended sections are on the tubular string. For the continuous contact bucking and helical buckling, the entire tubular string is divided into four suspended sections and a middle continuous contact section. In addition, the research method evolves from beam-column model to the combination of buckling differential equation and beam-column model. In a word, the buckling behaviors get more complicated and the calculation becomes more difficult with the transformation of buckling mode.

2. The starting point of continuous contact section is below the neutral point but above the point where axial force is equal to the critical continuous contact buckling or helical buckling load. For tubular strings of small diameter or thickness, such as tubing string, the difference among these three points can be neglected; However, for tubular strings of large diameter or thickness, the difference among these three points are obvious.

3. The coupling of buckling and friction force may greatly reduce the axial force and torque transfer along the tubular string and leads to serious buckling problem on the tubular string. Buckling can cause large loss of axial force and torque, and large loss of axial force and torque leads to buckling in turn. Stabilizer of low friction and buckling inhibition is an effective tool to overcome vicious circle. On one hand, installing stabilizers is wiser than unilaterally 
increasing the diameter and thickness of the tubular string to inhibit buckling, since excessive total tubular string weight increases the difficulty of pick-up operation. On the other hand, low friction factor between the tubular string and wellbore can reduce the friction drag between the tubular string and wellbore. Therefore, the next stabilizer design should be focused on the comprehensive effect of low friction and buckling inhibition.

4. In the sliding mode, WOB has its upper limit value even if the WSL tends to infinity. In the rotating mode, the minimum TOR to rotate the entire tubular string may exceed the maximum power of the drill rig or the torsional strength of the tubular string. Therefore, there are limits for down-hole tubular strings under a series of operations. The next research should be focused on the quantitative studies of extending limits of down-hole tubular strings.

\section{Acknowledgements}

The authors gratefully acknowledge the financial support from the Natural Science Foundation of China (Grant numbers: 51521063) and National Science Foundation for Distinguished Young Scholars of China (Grant numbers: 11325211). This research is also supported by national key research and development project (Grant numbers: 2016YFC0303303).

\section{Appendix A-Special functions of the general solutions}

The general solutions (Eq. (48)) are expressed in the form of special functions below. The definitions of the special functions including "hypergeom", "BesselI", "BesselJ", "AngerJ". "LommelS1", "WeberE" and "Gamma" can be found in mathematical handbook (Olver and Lozier et al., 2010) and Maple handbook.

$$
\begin{aligned}
& d_{1}(\eta)=\left\{\begin{array}{cr}
\eta \cdot \operatorname{hypergeom}\left(\left[\frac{1}{3}\right],\left[\frac{2}{3}, \frac{4}{3}\right],-\frac{1}{9} \eta^{3}\right) & \eta<0 \\
0 & \eta=0 \\
\frac{1}{27} \Gamma\left(\frac{2}{3}\right) 3^{2 / 3}\left(\begin{array}{cc}
9 \operatorname{BesselJ}\left(-\frac{1}{3}, \frac{2}{3} \eta^{3 / 2}\right) \eta^{3 / 2}+\eta^{3 / 2} \operatorname{BesselJ}\left(\frac{2}{3}, \frac{2}{3} x^{3 / 2}\right) \pi \sqrt{3} \cdot \operatorname{AngerJ}\left(\frac{5}{3}, \frac{2}{3} \eta^{3 / 2}\right) \\
+3 \eta^{3 / 2} \operatorname{BesselJ}\left(\frac{2}{3}, \frac{2}{3} \eta^{3 / 2}\right) \pi \cdot \operatorname{WeberE}\left(\frac{5}{3}, \frac{2}{3} \eta^{3 / 2}\right)+18 \operatorname{LommelS} 1\left(1, \frac{2}{3}, \frac{2}{3} \eta^{3 / 2}\right) \cdot \\
\operatorname{BesselJ}\left(\frac{2}{3}, \frac{2}{3} x^{3 / 2}\right)-9 \operatorname{LommelS} 1\left(1, \frac{2}{3}, \frac{2}{3} \eta^{3 / 2}\right) \cdot \operatorname{BesselJ}\left(-\frac{1}{3}, \frac{2}{3} \eta^{3 / 2}\right) \eta^{3 / 2}
\end{array}\right) & \eta>0
\end{array}\right.
\end{aligned}
$$

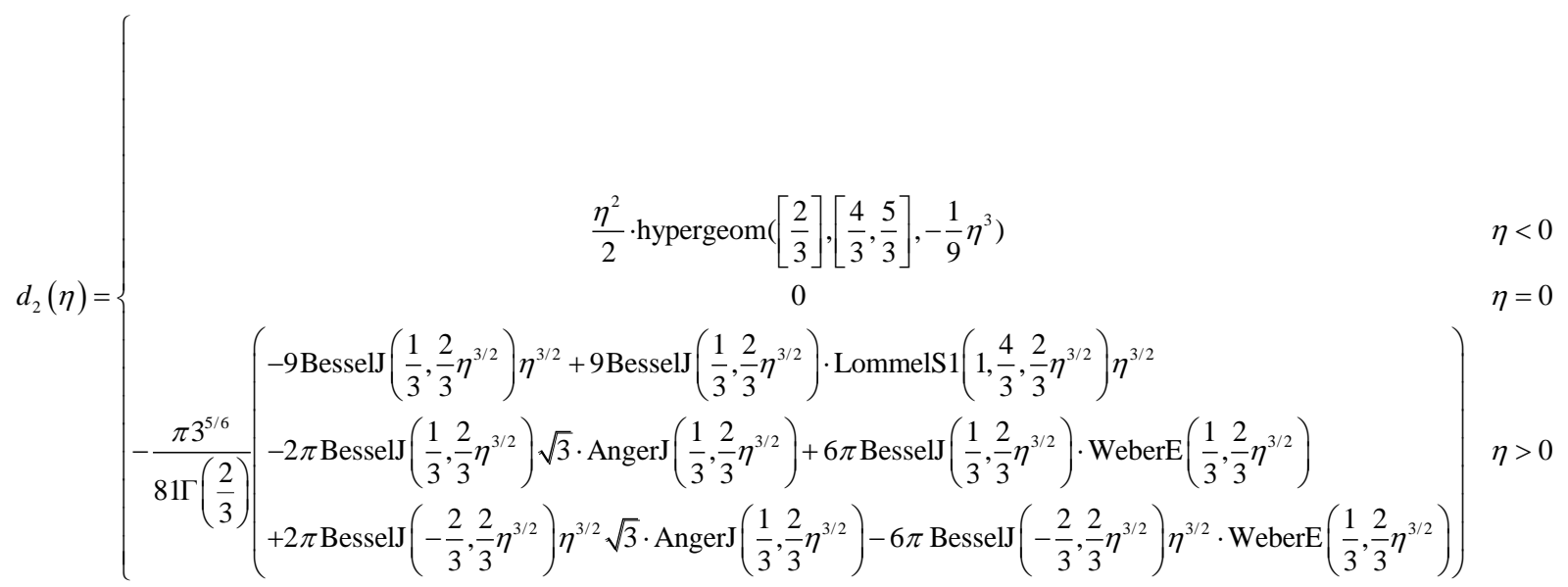

$$
\begin{aligned}
& d_{3}(\eta)=-\frac{\eta^{3}}{6} \cdot \text { hypergeom }\left([1,1],\left[\frac{4}{3}, \frac{5}{3}, 2\right],-\frac{1}{9} \eta^{3}\right)
\end{aligned}
$$




$$
d_{4}(\eta)=1
$$

$$
e_{1}(\eta)=\left\{\begin{array}{cc}
\frac{\sqrt{-\eta}}{3} \cdot \operatorname{BesselI}\left(-\frac{1}{3}, \frac{2}{3}(-\eta)^{3 / 2}\right) \Gamma\left(\frac{2}{3}\right) 3^{2 / 3} & \eta<0 \\
1 & \eta=0 \\
\frac{\sqrt{\eta}}{6} \cdot \operatorname{BesselI}\left(-\frac{1}{3}, \frac{2}{3} \mathrm{I} \eta^{3 / 2}\right) \Gamma\left(\frac{2}{3}\right) 3^{2 / 3}(\sqrt{3}+\mathrm{I}) & \eta>0
\end{array}\right.
$$

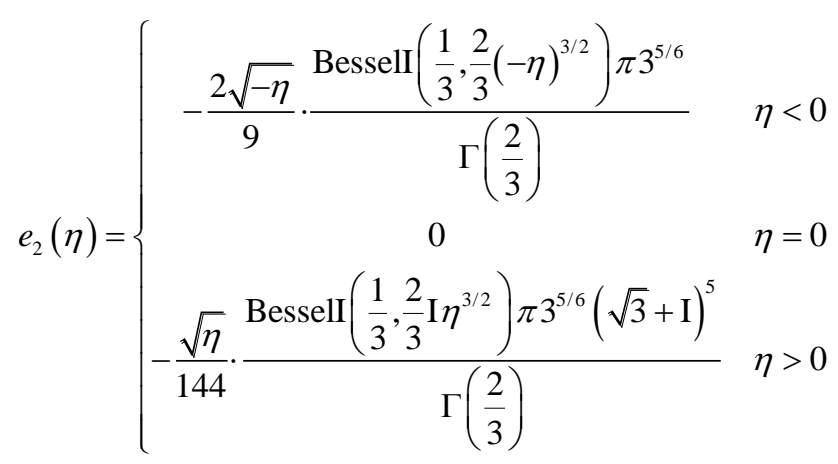

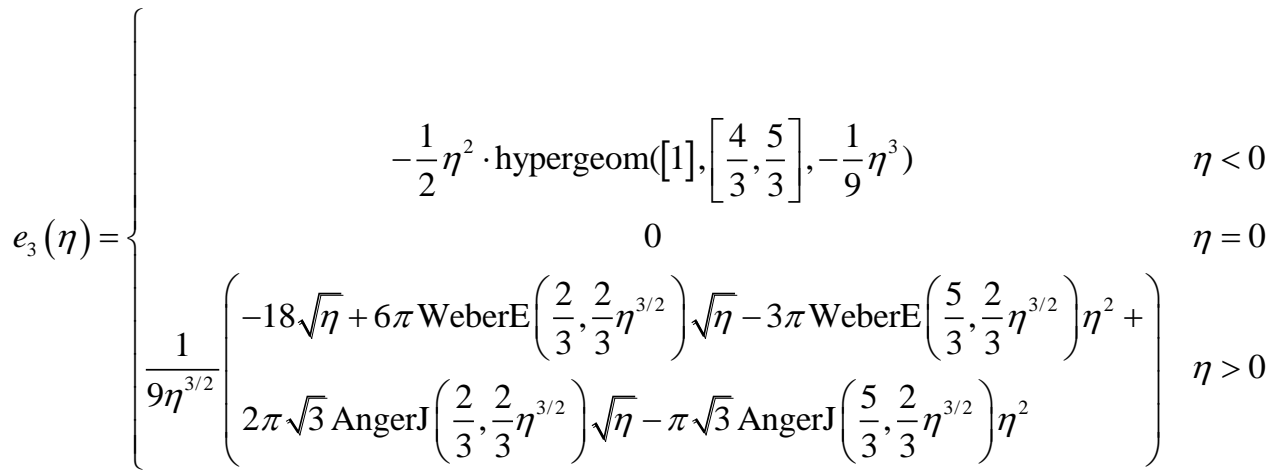

$$
e_{4}(\eta)=0
$$

$$
f_{1}(\eta)=\left\{\begin{array}{cc}
\frac{1}{3} \eta \cdot \operatorname{BesselI}\left(\frac{2}{3}, \frac{2}{3}(-\eta)^{3 / 2}\right) \Gamma\left(\frac{2}{3}\right) 3^{2 / 3} & \eta<0 \\
0 & \eta=0 \\
\frac{1}{12} \eta \cdot \operatorname{BesselI}\left(\frac{2}{3}, \frac{2}{3} \mathrm{I} \eta^{3 / 2}\right) \Gamma\left(\frac{2}{3}\right) 3^{2 / 3}(1+\mathrm{I} \sqrt{3})^{2} & \eta>0
\end{array}\right.
$$

$$
f_{2}(\eta)=\left\{\begin{array}{cc}
-\frac{2}{9} \eta \cdot \frac{\operatorname{BesselI}\left(-\frac{2}{3}, \frac{2}{3}(-\eta)^{3 / 2}\right) \pi 3^{5 / 6}}{\Gamma\left(\frac{2}{3}\right)} & \eta<0 \\
\frac{1}{9} \cdot \frac{\operatorname{BesselI}\left(-\frac{2}{3}, \frac{2}{3} \mathrm{I} \eta^{3 / 2}\right) \pi 3^{5 / 6}(1+\mathrm{I} \sqrt{3})}{\Gamma\left(\frac{2}{3}\right)} & \eta>0
\end{array}\right.
$$




$$
\begin{aligned}
& f_{3}(\eta)=\left\{\begin{array}{cc}
-\eta \cdot \operatorname{hypergeom}\left([1],\left[\frac{2}{3}, \frac{4}{3}\right],-\frac{1}{9} \eta^{3}\right) & \eta<0 \\
0 & \eta=0 \\
-\frac{1}{9} \eta \cdot \pi\left(3 \text { WeberE }\left(\frac{2}{3}, \frac{2}{3} \eta^{3 / 2}\right)+\sqrt{3} \operatorname{AngerJ}\left(\frac{2}{3}, \frac{2}{3} \eta^{3 / 2}\right)\right) & \eta>0
\end{array}\right. \\
& f_{4}(\eta)=0 \\
& g_{1}(\eta)=\left\{\begin{array}{cc}
\frac{1}{3} \sqrt{-\eta^{3}} \cdot \operatorname{BesselI}\left(-\frac{1}{3}, \frac{2}{3}(-\eta)^{3 / 2}\right) \Gamma\left(\frac{2}{3}\right) 3^{2 / 3} & \eta<0 \\
0 & \eta=0 \\
-\frac{1}{6} \eta^{3 / 2} \cdot \operatorname{BesselI}\left(-\frac{1}{3}, \frac{2}{3} \mathrm{I} \eta^{3 / 2}\right) \Gamma\left(\frac{2}{3}\right) 3^{2 / 3}(\sqrt{3}+\mathrm{I}) & \eta>0
\end{array}\right. \\
& g_{2}(\eta)=\left\{\begin{array}{cc}
-\frac{2}{9} \sqrt{-\eta^{3}} \cdot \frac{\operatorname{BesselI}\left(\frac{1}{3}, \frac{2}{3}(-\eta)^{3 / 2}\right) \pi 3^{5 / 6}}{\Gamma\left(\frac{2}{3}\right)} & \eta<0 \\
\frac{\eta^{3 / 2}}{144} \cdot \frac{\operatorname{BesselI}\left(\frac{1}{3}, \frac{2}{3} \mathrm{I} \eta^{3 / 2}\right) \pi 3^{5 / 6}(\sqrt{3}+\mathrm{I})^{5}}{\Gamma\left(\frac{2}{3}\right)} & \eta>0
\end{array}\right. \\
& g_{3}(\eta)=\left\{\begin{array}{cc}
-1 \cdot \text { hypergeom }\left([1],\left[\frac{1}{3}, \frac{2}{3}\right],-\frac{1}{9} \eta^{3}\right) & \eta<0 \\
-1 & \eta=0 \\
1-\frac{2}{3} \pi \text { WeberE }\left(\frac{2}{3}, \frac{2}{3} \eta^{3 / 2}\right)+\frac{1}{3} \eta^{3 / 2} \pi \text { WeberE }\left(\frac{5}{3}, \frac{2}{3} \eta^{3 / 2}\right) & \\
-\frac{2}{9} \pi \sqrt{3} \text { AngerJ }\left(\frac{2}{3}, \frac{2}{3} \eta^{3 / 2}\right)+\frac{1}{9} \eta^{3 / 2} \pi \sqrt{3} \text { AngerJ }\left(\frac{5}{3}, \frac{2}{3} \eta^{3 / 2}\right) & \eta>0
\end{array}\right. \\
& g_{4}(\eta)=0
\end{aligned}
$$

\section{Appendix B-Derivation of torque transfer}

By introducing the dimensionless term $\tau=\sqrt{2} \mu_{f} \delta_{c} \delta_{d}$, Eq. (141) is transformed into

$$
\frac{d m}{d \eta}+\frac{\tau}{4} \eta^{3 / 2} m=-\frac{\tau}{4 \sqrt{2}} \eta^{2}
$$

The general solution of Eq. (160) is expressed by 


$$
\begin{aligned}
m & =C \cdot e^{-\int \frac{\tau}{4} \eta^{3 / 2} d \eta}+e^{-\int \frac{\tau}{4} \eta^{3 / 2} d \eta} \cdot \int-\frac{\tau}{4 \sqrt{2}} \eta^{2} e^{\int \frac{\tau}{4} \eta^{3 / 2} d \eta} d \eta \\
& =C \cdot e^{-\frac{\tau}{10} \eta^{5 / 2}}-\frac{\tau}{4 \sqrt{2}} \cdot e^{-\frac{\tau}{10} \eta^{5 / 2}} \cdot \int \eta^{2} e^{\frac{\tau}{10} \eta^{5 / 2}} d \eta
\end{aligned}
$$

Where $C$ is the integral constant. The integral term $\int \eta^{2} e^{\frac{\tau}{10} \eta^{5 / 2}} d \eta$ can be written in the form of special function. Introducing a negative term $v=-\frac{\tau}{10} \eta^{5 / 2}$, we obtain

$$
\begin{aligned}
\int \eta^{2} e^{\frac{\tau}{10} \eta^{5 / 2}} d \eta & =-\frac{4}{\tau} \int \sqrt[5]{\frac{-10 v}{\tau}} e^{-v} d v \\
& =-\frac{4}{\tau}\left(\frac{10}{\tau}\right)^{\frac{1}{5}} \int \sqrt[5]{-v} e^{-v} d v \\
& =-\frac{4}{\tau}\left(\frac{10}{\tau}\right)^{\frac{1}{5}}(-1)^{-1 / 5} \int v^{1 / 5} e^{-v} d v
\end{aligned}
$$

Considering that Gamma function is defined by $\Gamma(a, z)=\int_{z}^{\infty} \mathrm{e}^{-t} t^{a-1} d t$, Eq. (162) can be written as

$$
\int \eta^{2} e^{\frac{\tau}{10} \eta^{5 / 2}} d \eta=-\frac{4}{\tau}\left(\frac{10}{\tau}\right)^{\frac{1}{5}}(-1)^{-1 / 5}\left[C-\Gamma\left(\frac{6}{5}, v\right)\right]
$$

Substituting Eq. (163) into Eq. (161) we obtain

$$
m=C \cdot e^{-\frac{\tau}{10} \eta^{5 / 2}}-\frac{1}{\sqrt{2}} \cdot\left(\frac{-10}{\tau}\right)^{\frac{1}{5}} e^{-\frac{\tau}{10} \eta^{5 / 2}} \Gamma\left(\frac{6}{5},-\frac{\tau}{10} \eta^{5 / 2}\right)
$$

By letting $m=m_{3}$ at the vertical depth of $\eta=\eta_{3}$, the integral constant is determined. Thus, the torque transfer expression is obtained:

$$
m=\frac{\sqrt{2}}{2}\left(-\frac{\tau}{10}\right)^{-1 / 5} \mathrm{e}^{-\frac{\tau}{10} \eta^{5 / 2}}\left[\Gamma\left(\frac{6}{5},-\frac{\tau}{10} \eta_{3}^{5 / 2}\right)-\Gamma\left(\frac{6}{5},-\frac{\tau}{10} \eta^{5 / 2}\right)\right]+m_{3} \mathrm{e}^{\frac{\tau}{10}\left(\eta_{3}^{5 / 2}-\eta^{5 / 2}\right)}
$$

\section{References}

Chen, Y. and Y. Lin, et al. (1990). "Tubing and Casing Buckling in Horizontal Wells (includes associated papers 21257 and 21308 )." SPE Journal of Petroleum Technology 42 (02): 140-141, 191.

Cunha, J. C. (2004). "Buckling of Tubulars Inside Wellbores: A Review on Recent Theoretical and Experimental Works." SPE Drilling \& Completion 01 (19): 13 - 19.

Gao, D. (2006). Down-Hole Tubular Mechanics and Its Applications. Dongying, China University of Petroleum Press.

Gao, D. and W. Huang (2015). "A review on down-hole tubular string buckling in well engineering." Petroleum Science 12: 443-457.

Gere, J. M. and B. J. Goodno (2008). Mechanics of Materials. Toronto Canada, CL-Engineering.

Huang, W. and D. Gao (2014). "Helical buckling of a thin rod with connectors constrained in a cylinder." International Journal of Mechanical Sciences 84: 189-198.

Huang, W. and D. Gao (2014). "Sinusoidal buckling of a thin rod with connectors constrained in a cylinder." Journal of 
Natural Gas Science and Engineering 18 (0): 237-246.

Huang, W. and D. Gao, et al. (2015). "Boundary Condition: A Key Factor in Tubular String Buckling." SPE Journal Preprint. Huang, W. and D. Gao, et al. (2015). "Buckling Analysis of Tubular Strings in Horizontal Wells." SPE Journal 20 (02): 405 416.

Huang, W. and D. Gao (2015). "Helical buckling of a thin rod with connectors constrained in a torus." International Journal of Mechanical Sciences 98: 14-28.

Lubinski, A. (1950). A Study of the Buckling of Rotary Drilling Strings, American Petroleum Institute.

Lubinski, A. (1977). "Fatigue of Range 3 Drill Pipe." Revue de l'Institut Français du Pétrole 32 (2): 209-232.

Lubinski, A. and W. S. Althouse (1962). "Helical Buckling of Tubing Sealed in Packers." Journal of Petroleum Technology 14 (6): 655-670.

Hajianmaleki M., Daily J. S., "Advances in Critical Buckling Load Assessment for Tubulars inside Wellbores", Journal of Petroleum Science and Engineering, 2014, 116, 136-144.

Mitchell, R. F. (1982). "Buckling Behavior of Well Tubing: The Packer Effect." Society of Petroleum Engineers Journal 22 (5): 616-624.

Mitchell, R. F. (1986). "Simple Frictional Analysis of Helical Buckling of Tubing." SPE Drilling Engineering 1 (6): $457-465$.

Mitchell, R. F. (1996). "Comprehensive Analysis of Buckling With Friction." SPE Drilling \& Completion 11 (3): $178-184$.

Mitchell, R. F. (1997). "Effects of Well Deviation on Helical Buckling." SPE Drilling \& Completion 12 (1): 63-70.

Mitchell, R. F. (1999). "A Buckling Criterion for Constant-Curvature Wellbores." SPE Journal 4 (4): 349-352.

Mitchell, R. F. (2000). "Helical Buckling of Pipe With Connectors in Vertical Wells." SPE Drilling \& Completion 15 (3): 162-166.

Mitchell, R. F. (2003). "Lateral Buckling of Pipe With Connectors in Horizontal Wells." SPE Journal 8 (2): $124-137$.

Mitchell, R. F. and Z. M. Stefan (2006). "Helical Buckling of Pipe With Connectors and Torque." SPE Drilling \& Completion 21 (2): 108-115.

Olver, F. W. and D. W. Lozier, et al., Eds. (2010). NIST Handbook of Mathematical Functions. Cambridge, UK., Cambridge University Pr.

Paslay, P. R. and D. B. Bogy (1964). "The Stability of a Circular Rod Laterally Constrained to Be in Contact With an Inclined Circular Cylinder." Journal of Applied Mechanics 31 (4): 605-610.

Sorenson, K. G. and J. J. B. Cheatham (1986). "Post-Buckling Behavior of a Circular Rod Constrained Within a Circular Cylinder." Journal of Applied Mechanics 53 (4): 929-934.

Timoshenko, S. P. and J. M. Gere, Eds. (1963). Theory of Elastic Stability. New York, Tata McGraw-Hill Education.

Wu, J. (1992). Buckling behavior of pipes in directional and horizontal wells. Texas, Texas A\&M University. PhD Dissertation.

Wu, J. and H. C. Juvkam-Wold (1995). "Coiled Tubing Buckling Implication in Drilling and Completing Horizontal Wells." SPE Drilling \& Completion 10 (01): 16-21.

Wu, J. and H. C. Juvkam-Wold (1995). "The Effect of Wellbore Curvature on Tubular Buckling and Lockup." Journal of Energy Resources Technology 117 (3): 214-218.

Gulyayev V I, Gaidaichuk V V, Solovjov I L, et al (2009). The buckling of elongated rotating drill strings. Journal of Petroleum Science \& Engineering, 67(3):140-148.

Mitchell, R. F. (1988). "New Concepts for Helical Buckling." SPE Drilling Engineering 3 (3): 303-310.

X.C. Tan and P.J. Digby (1993). Buckling of drill string under the action of gravity and axial thrust. International Journal of Solids and Structures, 30(19):2675-2691. 